\title{
LOCAL SMOOTH ISOMETRIC EMBEDDINGS OF LOW DIMENSIONAL RIEMANNIAN MANIFOLDS INTO EUCLIDEAN SPACES
}

\author{
GEN NAKAMURA AND YOSHIAKI MAEDA
}

\begin{abstract}
Local smooth isometric embedding problems of low dimensional Riemannian manifolds into Euclidean spaces are studied. Namely, we prove the existence of a local smooth isometric embedding of a smooth Riemannian 3-manifold with nonvanishing curvature into Euclidean 6-space. For proving this, we give a local solvability theorem for a system of a nonlinear PDE of real principal type.

To obtain the local solvability theorem, we need a tame estimate for the linearized equation corresponding to the given PDE, which is presented by two methods. The first is based on the result of Duistermaat-Hörmander which constructed the exact right inverse for linear PDEs of real principal type by using Fourier integral operators. The second method uses more various properties of Fourier integral operators given by Kumano-go, which seems to be a simpler proof than the above.
\end{abstract}

\section{INTRODUCTION}

In the study of isometric embeddings of a Riemannian $n$-manifold into Euclidean space, there is the classical question [Y]:

Given a Riemannian $n$-manifold $\left(M^{n}, d s^{2}\right)$, is there a local isometric embedding $\left(M^{n}, d s^{2}\right)$ into Euclidean space $\mathbf{R}^{N}$, where $N=n(n+1) / 2$ ?

In particular, it is well known that there always exists a local isometric embedding of $\left(M^{n}, d s^{2}\right)$ into $\mathbf{R}^{N}$, if $\left(M^{n}, d s^{2}\right)$ is real analytic (cf. [J]). Thus it is interesting to consider this question in the smooth category. In particular, we will obtain existence theorems for smooth local isometric embeddings for $n=2,3$. As a typical result, the following theorem is well known (cf. [J]).

Theorem A. Let $\left(M^{2}, d s^{2}\right)$ be a $C^{\infty}$ Riemannian 2-manifold and $p_{0} \in M$. If there is a point $p_{0} \in M$ such that the Gaussian curvature does not vanish at $p_{0}$, then there exists a local $C^{\infty}$ isometric embedding of a neighborhood $U_{0}$ of $p_{0}$ into $\mathbf{R}^{3}$.

In this paper, we shall prove the three dimensional analogue of the above theorem.

Received by the editors February 2, 1987 and, in revised form, January 4, 1988.

1980 Mathematics Subject Classification (1985 Revision). Primary 58G15; Secondary 35L60. 
Theorem B. Let $\left(M^{3}, d s^{2}\right)$ be a $C^{\infty}$ Riemannian 3-manifold. If there is a point $p_{0} \in M$ such that the curvature tensor $R\left(p_{0}\right)$ does not vanish, then there exists a local $C^{\infty}$ isometric embedding of a neighborhood $U_{0}$ of $p_{0}$ into $\mathbf{R}^{6}$.

We remark that Bryant-Griffiths-Yang [BGY] proved a special case of Theorem $B$ under the assumption:

$$
\operatorname{Sig} R\left(p_{0}\right) \neq(0,0),(0,1) \text {. }
$$

Here $\operatorname{Sig} R\left(p_{0}\right)$ denotes the signature of $R\left(p_{0}\right)$ viewed as a symmetric linear operator acting on the space of 2-forms (cf. $\S 2$ ). They first proved a Nash-Moser type theorem for a nonlinear PDE whose linearized PDE is either symmetric hyperbolic or strongly hyperbolic, and then as its application, they solved the local isometric embedding problem under the assumption $(*)$. On the other hand, without the assumption $(*)$ the linearized equation of the isometric embedding equation is merely of real principal type (cf. [BGY] and $\S 1$ ). Therefore, the key to the proof of Theorems A-B is to establish the local solvability of the nonlinear PDE whose linearized operator is a system of real principal type.

In our scheme, we can also recover the result of Lin [L].

Theorem C. Let $\left(M^{2}, d s^{2}\right)$ be a $C^{\infty}$ Riemannian 2-manifold and fix a point $p_{0} \in M$. Assume that the Gaussian curvature $K$ at $p_{0}$ of $M^{2}$ satisfies $K\left(p_{0}\right)=0$ and $d K\left(p_{0}\right) \neq 0$.

Then there exists a local $C^{\infty}$ isometric embedding of a neighborhood $U_{0}$ of $p_{0}$ into $\mathbf{R}^{3}$.

As an application of the following local solvability theorem, we shall give a unified proof of Theorems A-C.

Theorem D. Let $\Phi(u)$ be a $\mathbf{R}^{N}$ valued nonlinear partial differential operator of order $m$ acting on $C^{\infty}$ maps $u: \mathbf{R}^{n} \rightarrow \mathbf{R}^{N}$ with appropriate smoothness and boundedness conditions. Assume that $\Phi(u)$ is Fréchet differentiable in any Sobolev space of order greater than $m+n / 2$ and denote its derivative by $\Phi^{\prime}(u)$. Let $x_{0} \in \mathbf{R}^{n}$ and $u_{0} \in C^{\infty}\left(U_{0}, \mathbf{R}^{N}\right)$. Assume that there exists an open set $U$ such that $\Phi^{\prime}\left(u_{0}\right)$ is an $N \times N$-system of real principal type on $U$ and $U$ is pseudoconvex with respect to $\Phi^{\prime}\left(u_{0}\right)$ and has the transversality property for $\Phi^{\prime}\left(u_{0}\right)$ (see $\S 1$ for the precise definition). Then there exists a neighborhood $U_{1} \subset$ $U$ of $x_{0}, s_{0} \in \mathbf{Z}_{+}$, and $\eta>0$ such that for any $g \in C^{\infty}\left(U_{1}\right)$ with

$$
\left\|g-\Phi\left(u_{0}\right)\right\|_{H^{\mathrm{s}}\left(U_{1}\right)}<\eta,
$$

there exists $u \in C^{\infty}\left(\mathbf{R}^{n}, \mathbf{R}^{N}\right)$ satisfying

$$
\Phi(u)=g \text { in } U_{1} .
$$

As we shall see in Appendix B, our proof of Theorem D is based on the Nash-Moser type implicit function theorem. Since the linearized equation

$$
\Phi^{\prime}(u) v=h
$$


in an open neighborhood $U_{1}$ of $x_{0}$ does not have uniqueness of solutions, we cannot obtain the higher regularity estimate for solutions in an appropriate space by differentiating $(0.3)$. To avoid this difficulty, we construct an exact right inverse $Q(u)$ of $\Phi^{\prime}(u)$ with the so-called tame estimate, and modifying the Nash-Moser iteration scheme, we get a smooth solution $u$ of $(0.2)$.

The notions of real principal type and pseudoconvexity for linear PDEs are given in [DH] (cf. $\S 1$, Definition 1.3, (ii) and (iv) of this paper). In $\S 4$ combined with Appendix A, we give a construction of $Q(u)$ following the arguments of [H and DH]. However, it is troublesome to prove $Q(u)$ is a right inverse of $\Phi^{\prime}(u)$ in a common neighborhood $U_{1}$ of $x_{0}$ for any $u$ close to $u_{0}$ and get the tame estimate for $Q(u)$. For these proofs, we modified the arguments of [H and DH] so that the dependency of $Q(u)$ on $\Phi^{\prime}(u)$ can be easily seen. In Appendix (A.1), we give the natural form of the Hamiltonian vector field corresponding to a principal symbol of real principal type, which is similar to that of so-called straightening out Theorem in dynamical systems. Using this, we get a microlocal normal form for a real principal type operator under the assumptions of pseudoconvexity and the transversality condition in Appendix (A.III). This transversality condition in Theorem D can be defined as follows: Let $P$ be a system pseudodifferential operator defined on $U$ and let $p$ be the determinant of its principal symbol. Given a point $z_{0} \in T^{*} U-\{0\}$ and a Lagrangian subspace $\lambda$ at $z_{0}$, there must exist a Lagrangian subspace $\lambda$ at $z_{0}$ such that $\lambda$ is transversal to the tangent space of the fiber through $z_{0}$ and to $\left(\exp t H_{p}\right) *(\lambda)$, whenever the projected image of the Hamiltonian flow $\exp t H_{p}\left(z_{0}\right)$ through $z_{0}$ on the base space is contained in $U$. Although the transversality condition appears to be rather technical, it makes the proof of Theorem D clear and simple. Specifically, the transversality condition gives us the phase function on a conic neighborhood along a bicharacteristic needed to construct a parametrix $Q(u)$. In fact, we can relax the above condition in Theorem D as follows:

Theorem E. There exists an open set $U_{1} \subset \mathbf{R}^{n}$ such that the results of Theorem $\mathrm{D}$ hold without the transversality condition in Theorem $\mathrm{D}$.

In $\S 5$, we give a proof of Theorem $E$ different from that of Theorem D. The idea is to consider a time evolution operator exp it $P$ for a given partial differential operator $P$ by solving a hypberbolic differential equation. However, this method seems to work well only for real principal type PDEs.

Our proof of Theorem $\mathrm{D}$ is done microlocally and involves only a mild use of PDEs whose linearized equation is locally solvable via Fourier integral operators. On the other hand, using the local solvability of nonlinear, real principal type PDEs in the strong sense ( $\$ 1$. Remark), DeTurck and Yang [DY] showed the local existence of smooth metrics with nondegenerate prescribed curvature on 3-manifolds. Both [DY] and our paper can be treated as problems in $C^{\infty}$ local solvability for involutive systems. Thus, there are interesting geometrical PDEs among the $C^{\infty}$ locally solvable involutive systems. Theorems D-E and 
their generalizations should have application to some geometry. In particular, Einstein field equation and prescribed curvature equation with some interacting fields become the special type of double characteristics equations under certain assumptions. These problems will be discussed in a forthcoming paper.

Acknowledgment. Goodman-Yang [GY] prove similar results and give applications by a different approach. The authors would like to thank Professor Kazdan for informing us about the results of [GY] and other helpful advice. Also, we are grateful to the referee for the various suggestions on our manuscript. Finally, our special thanks go to Professor Rosenberg who kindly corrected our English.

\section{LINEARIZED PDE FOR THE ISOMETRIC EMBEDDING EQUATION}

Let $U\left(u^{1}, u^{2}, \ldots, u^{n}\right)$ be a coordinate neighborhood around the given point $x_{0} \in M$ which we take as the origin and sometimes denote by $p_{0}$. To obtain an isometric embedding of $(M, g)$ into $\mathbf{R}^{N}$, we must prove the existence of a solution to the following nonlinear PDE in an open neighborhood $U_{1} \subset U$ of $x_{0}$.

$$
\sum_{A=1}^{N} \frac{\partial x^{A}}{\partial u^{i}} \cdot \frac{\partial x^{A}}{\partial u^{j}}=g_{i j}\left(u^{1}, \ldots, u^{n}\right), \quad i, j=1, \ldots, n,
$$

where $\left(g_{i j}(u)\right)$ are the components of the Riemannian metric $g$ in $U$.

We shall consider the linearized PDE corresponding to (1.1); that is, let $x^{A}(u)$ be a local $C^{\infty}$ embedding of $U$ into $\mathbf{R}^{N}$ and consider the following PDE for the unknown functions $\left(u^{A}(u)\right)$ :

$$
\sum_{A=1}^{N} \frac{\partial x^{A}}{\partial u^{i}} \cdot \frac{\partial y^{A}}{\partial u^{j}}+\frac{\partial y^{A}}{\partial u^{i}} \cdot \frac{\partial X^{A}}{\partial u^{j}}=k_{i j}(u), \quad i, j=1, \ldots, n,
$$

where $\left(k_{i j}(u)\right)$ is a smooth symmetric contravariant 2-tensor on $U$.

Choosing a unit normal frame field $\left\{N_{\lambda}(u)\right\}_{\lambda=n+1, \ldots, N}$ on $U$, we put

$$
y^{A}(u)=\sum_{i=1}^{n} y^{i} \frac{\partial x^{A}}{\partial u^{i}}+\sum_{\lambda=n+1}^{N} y^{\lambda} N_{\lambda}^{A} .
$$

Denote the covariant derivatives and the second fundamental tensor for the isometric embedding $(1.1)$ by $\nabla_{i}$ and $H_{i j}^{\lambda}(u)$, respectively. Here, $\nabla_{i}$ is defined for a covariant 1-tensor $\xi_{j}(u)$ on $U$ as follows:

$$
\nabla_{i} \xi_{j}(u)=\partial_{u^{i}} \xi_{j}(u)-\sum_{k=1}^{n}\left\{\begin{array}{c}
k \\
i j
\end{array}\right\}(u) \xi_{k}(u)
$$

where $\left\{\begin{array}{c}k \\ i j\end{array}\right\}$ is the Christoffel tensor defined by

$$
\left\{\begin{array}{c}
k \\
i j
\end{array}\right\}=\frac{1}{2} \sum_{m=1}^{n} g^{k m}\left\{\partial_{u^{\prime}} g_{j m}+\partial_{u^{j}} g_{i m}-\partial_{u^{m}} g_{i j}\right\}
$$


Also, $H_{i j}^{\lambda}(u)$ is defined by the following Gauss-Weingarten equations associated with (1.1):

$$
\nabla_{j} \frac{\partial x^{A}}{\partial u^{i}}=\sum_{\lambda=n+1}^{N} H_{i j}^{\lambda} N_{\lambda}^{A}, \quad \nabla_{j} N_{\lambda}^{A}(u)=-\sum_{i=n+1}^{N} H_{j \lambda}^{i} \frac{\partial x^{A}}{\partial u^{i}}
$$

where the raising and lowering of indices are with respect to the given Riemannian metric. Rewriting (1.2) by (1.4), we easily get

Lemma 1.1.

$$
\nabla_{i} y_{j}+\nabla_{j} y_{i}=2 \sum_{\lambda=n+1}^{N} y_{\lambda} H_{i j}^{\lambda}(u)+k_{i j}(u), \quad i, j=1, \ldots, n .
$$

Definition 1.2. (i) The second fundamental tensor $\left(H_{i j}^{\lambda}\left(x_{0}\right)\right)_{\lambda=n+1, \ldots, N}$ is called nondegenerate at $x_{0}$ if $\left\{\left(H_{i j}^{\lambda}\right)\left(x_{0}\right)\right\}$ are linearly independent in the vector space of all covariant symmetric 2-tensors at $x_{0}$.

(ii) An isometric embedding is called nondegenerate if the corresponding second fundamental form is nondegenerate at each point of $U$.

Although the second fundamental tensor $H_{i j}^{\lambda}(u)$ depends on choosing the normal frame $N_{\lambda}^{A}(u)$, the above definition is independent of this choice.

Next we recall some PDE terminologies. Let $T^{*} M$ denote the cotangent bundle of $M$. For a positive integer $N$, let $P$ be an $N \times N$ system of classical pseudodifferential operators on $M$ with principal symbol $p(x, \xi)$.

Definition 1.3. (i) $P$ is called a system of real principal type at $\left(x_{0}, \xi_{0}\right) \in T^{*} M-$ $\{0\}$ if there exists a conic neighborhood $\Gamma$ of $\left(x_{0}, \xi_{0}\right)$, an $N \times N$ classical symbol $\tilde{p}(x, \xi)$, and a real valued classical scalar symbol $q(x, \xi)$ such that

$$
\tilde{p}(x, \xi) p(x, \xi)=q(x, \xi) \operatorname{Id}_{N} \quad \text { in } \Gamma,
$$

and the principal symbol $q_{1}(x, \xi)$ of $q(x, \xi)$ satisfies the condition that $d q_{1}$ and $\theta=\sum_{i=1} \xi_{i} d x^{i}$ are linearly independent on $\Gamma \cap\left\{(x, \xi) ; q_{1}(x, \xi)=0\right\}$.

Here $\operatorname{Id}_{N}$ is the $N \times N$ identity matrix.

(ii) $P$ is called a system of real principal type at $x_{0} \in M$ if $P$ is a system of principal type at each point of $\pi^{-1}\left(x_{0}\right)-\{0\}$, where $\pi: T^{*} M \rightarrow M$ is the projection.

(iii) $P$ is called a system of real principal type on an open set $U$ of $M$ if $P$ is a system of principal type at each point of $\pi^{-1}(U-\{0\})$.

(iv) Let $P$ be of real principal type on an open set $U$ and associate a scalar symbol $q_{1}$ to $p$ given in (i). We say that $U$ is pseudoconvex with respect to $P$ if no complete bicharacteristic strip of $P$ stays over a compact set in $U$ and for every compact set $K \subset U$ there is another compact set $K^{\prime} \subset U$ such that every bicharacteristic interval with respect to $P$ having endpoints over $K$ must lie entirely over $K^{\prime}$. 
(v) Let $P$ be a system of pseudodifferential operators on an open set $U$ and associate a scalar symbol $q_{1}$ to $p$ given in (i). Assume that $U$ is pseudoconvex with respect to $P . U$ is said to have the transversal property for $P$, if the following holds: for any point $z_{0} \in{ }^{o} T^{*} U \equiv T^{*} U-\{0\}$ and any Lagrangian subspace $\lambda$ at $z_{0}$, there exists a Lagrangian subspace $\lambda^{\perp}$ at $z_{0}$ such that $\lambda^{\perp}$ is transversal to the tangent space of the fiber through $z_{0}$ and to $\left(\exp t H_{q_{1}^{*}}\right)(\lambda)$, whenever $\pi\left(\exp t H_{q_{1}}\left(z_{0}\right)\right)$ is contained in $U$. Here $\exp t H_{q_{1}}$ is the Hamiltonian flow of the Hamiltonian vector field $H_{q_{1}}$ (cf. Appendix A, (A.6)) and $\left(\exp t H_{q_{1}}^{*}\right)(\lambda)$ is identified with the subspace at $z_{0}$.

Remark. (i) The conditions in (i)-(v) for the principal symbol $q_{1}$ of $P$ obviously hold if $\operatorname{grad}_{\xi} q_{1} \neq 0$ on $\Gamma \cap\left\{(x ; \xi) ; q_{1}(x, \xi)=0\right\}$. In this case we say that $P$ is a system of real principal type at $\left(x_{0}, \xi_{0}\right) \in T^{*} M-\{0\}$ in the strong sense. By modifying Definition 1.3, (ii) in an obvious manner, we obtain the corresponding definitions at a point $x_{0} \in M$ and on an open set $U$ of $M$.

(ii) As we will see in Proposition 2.2, the linearized PDE of the isometric embedding equation is not real principal type in the strong sense only in the case of Theorem $\mathrm{C}$.

Corresponding to Definition 1.2, we have

Definition 1.4. (i) The isometric embedding (1.1) is called a real principal type at $x_{0} \in M$ (resp. on $U$ ) if the linearized equation (1.5) is of real principal type at $x_{0}$ (resp. on $U$ ).

(ii) For a real principal type isometric embedding (1.1) on $U$, we say that $U$ is a pseudoconvex for (1.1) if $U$ is pseudoconvex for the linearized equation (1.5).

Before constructing a nondegenerate, principal type embedding in $\S 2$, we study a second fundamental tensor at a fixed point $x_{0}$. First, we recall that the Gauss equation for the isometric embedding gives the constraint

$$
R_{i j k l}(u)=\sum_{\lambda=n+1}^{N} H_{i l}^{\lambda}(u) \cdot H_{i j}^{\lambda}(u)-H_{i k}^{\lambda}(u) \cdot H_{j l}^{\lambda}(u)
$$

for the desired isometric embedding.

Now we recall the following fact obtained by [BGY] (see p. 948, Theorem F).

Proposition 1.5. Let $\left(M^{n}, d s^{2}\right)$ be a Riemannian $n$-manifold $(n \leq 3)$, which satisfies the assumptions in Theorems A-B. Then, there exists an $(N-n)$-tuple of $n \times n$ symmetric matrix $\left(H_{i j}^{\lambda}(0)\right)_{\lambda=n+1, \ldots, N}$ such that

(i) for $\left(H_{i j}^{\lambda}(0)\right)_{\lambda=n+1, \ldots, N},(1.5)$ is real principal type at the origin $0 \in \mathbf{R}^{n}$.

(ii) $\left(H_{i j}^{\lambda}(0)\right)_{\lambda=n+1, \ldots, N}$ satisfies (1.7) at the origin $0 \in \mathbf{R}^{n}$. Furthermore,

(iii) $\left(H_{i j}^{\lambda}(0)\right)_{\lambda=n+1, \ldots, N}$ is nondegenerate at the origin $0 \in \mathbf{R}^{n}$. 


\section{CONSTRUCTION OF A REAL PRINCIPAL TYPE EMBEDDING}

In this section we shall construct a real principal type embedding whose induced metric is sufficiently close to the given Riemannian metric $d s^{2}$. Namely, we prove the following:

Proposition 2.1. Let $\left(M^{n}, d s^{2}\right)$ be a $C^{\infty}$ Riemannian manifold with $n=2$ or $n=3$ and $\left(H_{i j}^{\lambda}\right)_{\lambda=n+1, \ldots, N}$ be an $(N-n)$-tuple matrix satisfying the properties in Proposition 1.5. Under the assumptions of Theorems A-B, given any $\eta>0$ and any positive integer $s$, there exist an open neighborhood $U_{0}$ of $p_{0}$, an $(N-n)$-tuple of local $C^{\infty}$ unit vector fields $\left(N_{\lambda}^{A}(u)\right)_{\lambda=n+1, \ldots, N}$ on $U_{0}$, and a nondegenerate $C^{\infty}$ embedding $\left(x^{A}(u)\right)_{A=1, \ldots, N}$ of $U_{0}$ into $\mathbf{R}^{N}$ with $N=$ $n(n+1) / 2$ such that

(i) $\left(N_{\lambda}^{A}(u)\right)_{\lambda=n+1, \ldots, N}$ is a unit normal frame field on $U_{0}$ for the embedding $\left(x^{A}\right)_{A=1, \ldots, N}$.

(ii) $\left(H_{i j}^{\lambda}\right)$ is the second fundamental form of $\left(x^{A}\right)$ at $p_{0}$ with respect to $\left(N_{\lambda}^{A}(u)\right)$.

(iii) The embedding $x^{A}(u)$ is of real principal type on $U_{0}, U_{0}$ is pseudoconvex with respect to the linearized equation (1.5) and $U_{0}$ has the transversal property for (1.5).

(iv) $\left\|g_{i j}(u)-\sum_{A=1}^{N} \partial x^{A} / \partial u^{i} \cdot \partial x^{A} / \partial u^{j}\right\|_{H} \quad s\left(U_{0}\right)<\eta$.

To prove Proposition 2.1, we expand the Riemannian metric $g$ into its Taylor series by using normal coordinates $\left(u^{i}\right)$. Namely, let $g(u)=\left(g_{i j}(u)\right)$ be the Riemannian metric in the coordinate neighborhood $U\left(u^{1}, \ldots, u^{n}\right)$. We have the Taylor expansion of $g(u)$ up to degree $k$ :

$$
g_{i j}^{(k)}(u)=\delta_{i j}+\frac{1}{2 !} \sum A_{i j \alpha_{1} \alpha_{2}} u^{\alpha_{1}} u^{\alpha_{2}}+\cdots+\frac{1}{k !} \sum A_{i j \alpha_{1}, \ldots, \alpha_{k}} u^{\alpha_{1}} \cdots u^{\alpha_{k}} .
$$

Now we seek the mapping $i: U\left(u^{1}, \ldots, u^{n}\right) \rightarrow \mathbf{R}^{N}$ in the following form:

$$
\begin{aligned}
& i\left(u^{1}, \ldots, u^{n}\right)=\left(x^{(k) A}(u)\right) \\
& \quad=\sum I_{a}^{A} u^{a}+\frac{1}{2 !} \sum I_{a b}^{A} u^{a} u^{b}+\cdots+\frac{1}{k !} \sum I_{a_{1}, \ldots, a_{k}}^{A} u^{a_{1}} \cdots u^{a_{k}}
\end{aligned}
$$

satisfying

(H-I) $i(0)=0$.

(H-II) $d i(0)=\mathrm{Id}$.

(H-III) $N_{\lambda}(0)=(0, \ldots, \stackrel{(\lambda)}{1}, 0, \ldots, 0),(\lambda=n+1, \ldots, N)$.

(H-IV) Given nondegenerate $\left(H_{i j}^{\lambda}\right)$ satisfying (1.7), we have $H_{i j}^{\lambda}(0)=H_{i j}^{\lambda}$.

We solve (2.2) using the following relation:

$$
\sum_{A} \frac{\partial x^{(k) A)}}{\partial u^{i}} \cdot \frac{\partial x^{(i) A}}{\partial u^{j}}=g_{i j}(u) \bmod O\left(|u|^{k}\right) .
$$


Therefore, we have

$$
I_{a}^{A}=\delta_{a}^{A} \quad(a=1, \ldots, n) .
$$

Using $\left\{\begin{array}{c}a \\ b c\end{array}\right\}(0)=0$, we have

$$
I_{c a}^{\mu}=H_{c a}^{\mu} \quad(\mu=n+1, \ldots, N), \quad I_{c a}^{b}=0 \quad(b=1, \ldots, n) .
$$

Substituting (2.1) and (2.2) into (2.3) and equating the corresponding coefficients, we can solve (2.3) formally. Note that the equations thus obtained for the unknown $I^{A}$ are underdetermined. Thus only the symmetric part of each $I^{A}$ is restricted and we may choose the antisymmetric part arbitrarily. By the proof of Proposition 1.5, there exists an open set $U$ such that $\partial_{\xi} q_{1}(x, \xi) \neq 0$ on $U$, where

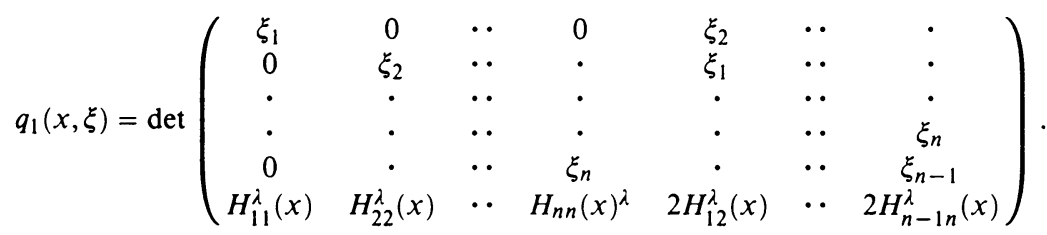

Since in this case, the linearized equation (1.5) is of real principal type in the strong sense, we get Proposition 2.1.

For the case of Theorem $\mathrm{C}$, we refine the argument above to obtain

Proposition 2.2. Let $\left(M^{2}, d s^{2}\right)$ be a Riemannian 2-manifold satisfying the assumptions in Theorem C. Given any $\eta>0$ and any positive integer $s$, there exists an open neighborhood $U_{0}$ of $p_{0}$ in $M$ with coordinates $\left(u^{1}, u^{2}\right)$ and a $C^{\infty}$ embedding $\left(x^{A}(u)\right)_{A=1,2,3}$ of $U_{0}$ into $\mathbf{R}^{3}$ such that (i) $\left(x^{A}(u)\right)$ is nondegenerate and of real principal type on $U_{0}$ with respect to the induced metric from $\mathbf{R}^{3}$ and $U_{0}$ is pseudoconvex with respect to the linearized equation (1.5) of $x^{A}(u)$ and has the transversal property. Moreover, we have

(ii) $\left\|g_{i j}(u)-\sum_{A=1}^{3} \partial x^{A} / \partial u^{i} \cdot \partial x^{A} / \partial u^{j}\right\|_{H^{s}\left(U_{0}\right)}<\eta$.

Proof. Take normal coordinates $\left(v^{1}, v^{2}\right)$ around $p_{0}$. Then we get the Taylor expansion $g_{i j}^{(k)}(v)$ of $g_{i j}(v)$ as in $(2.1)$ for $k$ sufficiently large. Notice that the Gaussian curvature $K^{(k)}(v)$ of $g_{i j}^{(i)}$ satisfies the same assumptions as in Theorem $\mathrm{C}$. Then by a linear change of coordinates $u^{i}=\sum_{j=1}^{2} A_{j}^{i} v^{j} \quad(i=1,2)$ $\left(A_{j}^{i}\right) \in O(2)$, the Gaussian curvature $K(u)$ can be expressed as

$$
K(u)=a u^{1}+O\left(|u|^{2}\right)
$$

with $a \neq 0$. Now we set

$$
H_{11}(0)=0, \quad H_{22}(0)=a, \quad H_{12}(0)=0 .
$$

Then by a computation as in Proposition 2.1 , we get a local $C^{\infty}$ embedding $x^{A}(u)$ which is nondegenerate and satisfies the condition (ii) in Proposition 2.2. 
Moreover, we can see that this embedding is of real principal type as follows. $q_{1}(u, \xi)$ of $(1.6)$ for the linearized PDE (1.5) is given by

$$
q_{1}(u, \xi)=H_{22}(u) \xi_{1}^{2}+H_{11}(u) \xi_{2}^{2}-2 H_{12}(u) \xi_{1} \xi_{2} .
$$

Notice that $q_{1}(0, \xi)=0$ implies $\xi_{1}=0$. By (2.7), we get

$$
\partial_{u^{\prime}} H_{11}(0) \neq 0 \text {. }
$$

Assume that there exists a nontrivial constant $k$ such that

$$
D q_{1}=k \theta \text {, }
$$

at $u=0, \xi_{1}=0, \xi \neq 0$. By using (2.10) and (2.11), we have

$$
\xi_{2}=0 \text {. }
$$

Then we easily see that $q_{1}$ and the canonical 1 -form $\theta$ are linearly independent at $u=0, \xi_{1}=0, \xi \neq 0$. Since the Gaussian curvature $K(u)$ does not vanish on a open set $U-\{0\}, \partial_{\xi} q_{1}(u, \xi) \neq 0$ on $\pi^{-1}(U-\{0\})$. Remark that the projected vector field of $H_{q_{1}}$ on the unit sphere bundle does not vanish and $\partial_{\xi} q_{1}(0, \xi) \neq 0$ for $\xi_{1} \neq 0$. The pseudoconvexity and transversality conditions easily follow. This gives the desired result.

Remark. Under the hypothesis in Proposition 2.1, the linearized equation corresponding to $\left(x^{A}(u)\right)$ is a real principal type in the strong sense. (In fact, [BGY] calls it a real principal type simply.) By combining with the result on the local existence of the analytic isometric embeddings (cf. [J]) and Proposition 1.5, Proposition 2.1 is obtained directly. However, the hypothesis in Proposition 2.2 is slightly weaker than the above, and so their argument does not work in a similar way.

\section{LOCAL SOLVABILITY OF REAL PRINCIPAL TYPE PARTIAL DIFFERENTIAL SYSTEMS}

We first give a general theorem on the local solvability of a nonlinear partial differential operator.

Let $U_{0} \subset \mathbf{R}^{n}$ be an open neighborhood of $x_{0} \in \mathbf{R}^{n}$ and $u_{0}: U_{0} \rightarrow \mathbf{R}^{N}$ be a $C^{\infty}$ map, (Hereafter, we also denote by $u_{0}$ a $C^{\infty}$ map from $\mathbf{R}^{n} \rightarrow$ $\mathbf{R}^{N}$ obtained by cutting off the support of $u_{0}$.) Let $\Phi(u)=\Phi\left(x, \bar{D}^{m} u\right)$ be a $\mathbf{R}^{N}$-valued nonlinear partial differential operator of order $m$ applied to $u \in$ $C^{\infty}\left(\mathbf{R}^{n}, \mathbf{R}^{N}\right)$, where

$$
\begin{gathered}
\bar{D}^{m} u=\left(D^{\alpha} u ;|\alpha| \leq m\right), \quad D^{\alpha}=D_{1}^{\alpha_{1}} \cdots D_{n}^{\alpha_{n}}, \\
D_{i}=-\sqrt{-1} \frac{\partial}{\partial x_{i}}, \quad \alpha=\left(\alpha_{1}, \ldots, \alpha_{n}\right) .
\end{gathered}
$$

Theorem 3.1. In the above situation, assume that

$$
\Phi(x, w) \in B^{\infty}\left(\mathbf{R}^{n} \times\left\{|w| \leq w_{0}\right\}\right) \text { for any } w_{0}>0 ;
$$


this assures the Fréchet differentiability of $\Phi(u)$ with respect to $u \in H^{s}\left(\mathbf{R}^{n}\right)$, $(s>m+n / 2)$. Assume also that there exist an open neighborhood $U_{1} \subset U_{0}$ of $x_{0} \in \mathbf{R}^{n}$ with a smooth boundary $\alpha>m+n / 2, \delta \in(0,1)$ and $d \geq 0$ such that, for any $u\left(\left\|u-u_{0}\right\|_{\alpha} \leq \delta\right), s \in \mathbf{R}$ and $h \in H^{s}\left(\mathbf{R}^{n}\right)$, the equation

$$
\Phi^{\prime}(u) v=h \quad \text { in } U_{1}
$$

admits a solution $v \in H^{s-d}\left(\mathbf{R}^{n}\right)$ with the so-called tame estimate

$$
\|v\|_{s-d} \leq C_{s}\left(\|h\|_{s}+\|u\|_{s}\|h\|_{d}\right),
$$

where $C_{s}$ is independent of $u$ and $h$. Then, there exists $s_{0} \in \mathbf{Z}_{+}$and $\eta>0$ such that, for any $g \in C^{\infty}\left(U_{1}\right)$ with

$$
\left\|g-\Phi\left(u_{0}\right)\right\|_{s_{0}}^{0} \equiv\left\|g-\Phi\left(u_{0}\right)\right\|_{H^{s_{0}\left(U_{1}\right)}}<\eta
$$

the equation

$$
\Phi(u)=g \quad \text { in } U_{1}
$$

admits a solution $u \in C^{\infty}\left(\mathbf{R}^{n}, \mathbf{R}^{N}\right)$. Here $B^{\infty}\left(\mathbf{R}^{n} \times\left\{|w| \leq w_{0}\right\}\right)$ denotes the set of all $\mathbf{R}^{N}$-valued $C^{\infty}$ functions with bounded derivatives.

The proof is given in Appendix $B$.

Theorem 3.2. Let $x_{0}, U_{0}, \Phi(u)$ be as in Theorem 3.1 with all the assumptions in Theorem 3.1 except for (3.1), (3.2). Suppose the Fréchet derivative $\Phi^{\prime}\left(u_{0}\right)$ of $\Phi(u)$ at $u_{0}$ is a system of real principal type on $U_{0}$ and $U_{0}$ is pseudoconvex with respect to $\Phi^{\prime}\left(u_{0}\right)$ and has the transversality property for $\Phi^{\prime}\left(u_{0}\right)$. Then there exist an open neighborhood $U_{1} \subset U_{0}$ of $x_{0}$ with smooth boundary, $s_{0} \in \mathbf{Z}_{+}$and $\eta>0$ such that, for any $g \in C^{\infty}\left(U_{1}\right)$ with $\left\|g-\Phi\left(u_{0}\right)\right\|_{H^{s_{0}}\left(U_{1}\right)}<\eta$, the equation (3.3) admits a solution $u \in C^{\infty}\left(\mathbf{R}^{n}, \mathbf{R}^{N}\right)$.

Proof of Theorem 3.2. From Theorem 3.1, it is enough to prove the existence of $U_{1}, \alpha, \delta, d$ satisfying (3.1), (3.2), (3.3). To begin with we set

$$
L(u)=\Phi^{\prime}(u)
$$

$$
l(x, \xi ; u)=\text { the principal symbol of } \operatorname{det} L(x, \xi ; u),
$$

and

$$
\begin{array}{r}
M(x, D ; u) \equiv L(x, D ; u)^{c o} L(x, D ; u)=l(x, D ; u) I+R(x, D ; u), \\
R(x, D ; u) \in S^{m N-1},
\end{array}
$$

where $S^{m N-1}$ denotes the usual Hörmander class $S_{1,0}^{m N-1}$. We have the following lemma for the local solvability of the operator $(3.6)$. 
Lemma 3.3. Suppose there are an open neighborhood $U_{1} \subset U_{0}$ of $x_{0}, m+n / 2<$ $d_{1}, d_{2} \in \mathbf{Z}_{+}, d^{\prime}=\max \left(d_{1}, d_{s}\right)<\alpha^{\prime} \in \mathbf{Z}_{+}$, and $\delta_{1}>0$ such that for any $u$ $\left(\left\|u-u_{0}\right\|_{\alpha^{\prime}} \leq \delta_{1}\right)$, there exist an operator $Q(u)$ and $\phi_{j} \in C_{0}^{\infty}\left(U_{0}\right) \quad(1 \leq j \leq 3)$ with the properties:

$$
\begin{gathered}
\phi_{1}=1 \quad \text { in } U_{1}, \\
\phi_{1} \subset \phi_{2} \subset \phi_{3}, \\
\|Q(u) h\|_{s-d_{1}} \leq C_{s}\left(\|h\|_{s}+\|u\|_{s}\|h\|_{d_{1}}\right) \quad\left(d_{1} \leq s \in \mathbf{Z}_{+} ; h \in H^{s}\left(\mathbf{R}_{n}\right),\right. \\
\|K(u)\| \leq \frac{1}{2},
\end{gathered}
$$

$$
\left\|(1-K(u))^{-1} h\right\|_{s} \leq C_{s}\left(\|h\|_{s}+\|u\|_{s}\|h\|_{d_{2}}\right) \quad\left(s \in \mathbf{Z}_{+} ; h \in H^{s}\left(\mathbf{R}^{n}\right)\right),
$$

where

$$
K(u)=\phi_{2}(I-M(u) Q(u)) \phi_{3} .
$$

Here $\|K(u)\|$ denotes the operator norm of $K(u): L^{2}\left(\mathbf{R}^{n}\right) \rightarrow L^{2}\left(\mathbf{R}^{n}\right)$ and $\phi_{1} \subset$ $\phi_{2}$ means that $\phi_{2}=1$ on $\operatorname{supp} \phi_{1}$. Then,

$$
\begin{gathered}
\phi_{1}\left\{M(u) Q(u)(1-K(u))^{-1} Q_{2}-I\right\}=0, \\
\left\|Q(u)(1-K(u))^{-1} \phi_{2} h\right\|_{s-d^{\prime}} \leq C_{s}\left(\|h\|_{s}+\|u\|_{s}\|h\|_{d^{\prime}}\right) \\
\left(d^{\prime} \leq s \in \mathbf{Z}_{+} ; h \in H^{s}\left(\mathbf{R}^{n}\right)\right),
\end{gathered}
$$

and these immediately imply the following: for any $u\left(\left\|u-u_{0}\right\|_{\alpha} \leq \delta_{1}\right)$, we have

$$
\phi_{1}\left[L(u)\left\{{ }^{c o} L(u) Q(u)(1-K(u))^{-1} \phi_{2}\right\}-I\right]=0
$$

and

$$
\begin{array}{r}
\left\|^{c o} L(u) Q(u)(1-K(u))^{-1} \phi_{2} h\right\|_{s-d} \leq c_{s}\left(\|h\|_{s}+\|u\|_{s}\|h\|_{d}\right) \\
\left(d \leq s \in \mathbf{Z}_{+} ; h \in H^{s}\left(\mathbf{R}^{n}\right)\right),
\end{array}
$$

where

$$
\begin{gathered}
\alpha=\alpha^{\prime}+[n / 2]+1, \\
d=d^{\prime}+\max \{m N-1, m+[n / 2]+1\} .
\end{gathered}
$$

The proof of Lemma 3.3 is by direct computation, so we omit the proof.

Now to complete the proof of Theorem 3.2, we only have to construct the operator $Q(u)$ in Lemma 3.3. This is done in $\S 4$.

Remark. The assumption of transversality in Theorem 3.2 can be dropped if we use $\S 5$.

Finally, we shall make a brief comment on the unified proof of Theorems A-C stated in the introduction. Recall that we already have obtained in $\S 2$ 
a real principal type embedding which is close to a given Riemannian metric $g$ in an open neighborhood $U_{0}$ of $p_{0}$ and satisfies the pseudoconvexity and the transversality properties for $U_{0}$. Thus Theorem 3.2 immediately implies Theorems A-C.

\section{Proof of Theorem D}

In this section, we give the proof of Theorem D. To show this, we need several preliminaries for symplectic geometry and formulas for Fourier integral operators. Leaving these in Appendix A, we mention how to prove Theorem D. Namely, we often use the results of Proposition A.4 and Theorem A.10. What we want to get is the construction of a local right inverse of the real principal type linear PDE of the form

$$
P u=f
$$

under the assumptions of Theorem D and the estimate (3.2) in Theorem 3.1, which is the key estimate for our iteration scheme stated in $\S 3$. Our method follows the work of Hörmander [H] and Duistermaat-Hörmander [DH].

To use our iteration scheme, we have to estimate the dependence of the local right inverse on $P$. However, the method given in [H and $\mathrm{DH}]$ is too complicated to analyze this dependency. The main part of this construction is to reduce the operator $P$ in $(*)$ to a canonical one (cf. Propositions A.4, A.5 and Theorem A.10), by combining a geometrical idea based on the proof of Darboux's lemma (cf. [M, W and $\mathrm{AB}]$ ) with a direct and routine computation using Fourier integral operators. We will also obtain the continuous dependence of $p$ on the symbol function $p(x, \xi)$ of $P$. These arguments are technically similar to [OMY1-2 and OMYK3-7].

Here, for later reference, we list the assumptions of Theorem D.

(*1) $P$ is a real principal type on a open set $U$.

(*2) $U$ is pseudoconvex with respect to $P$.

(*3) $U$ has the transversal property for $P$.

First, we make some remarks about the fundamental solution of the operator $D_{1}=(1 / i) \partial / \partial x^{1}$ on $\mathbf{R}^{n}$. Let $W_{2}$ be a relatively compact open set of $\mathbf{R}^{n}$ which contains the origin and let $W_{1}$ be a sufficiently small open subset of $W_{2}$ such that $0 \in W_{1} \subset W_{2}$. Denote the fundamental solution of $D_{1}$ on $\mathbf{R}^{n}$ by $E_{1}(x, y)$, i.e.

$$
E_{1}(x, y)=i H\left(x^{1}-y^{1}\right) \otimes \delta\left(x^{\prime}-y^{\prime}\right) .
$$

Here, $H(t)$ is the Heaviside function, $H(t)=1$ on $t>0$ and $H(t)=0$ for $t<0$, and we have used the notation $x=\left(x^{1}, x^{\prime}\right)$ and $\left(y^{1}, y^{\prime}\right)$. Let $\lambda(t)$ be a $C^{\infty}$ function satisfying

$$
\lambda(t) \equiv 1 \quad \text { on }|t| \leq \frac{1}{3} r_{0}, \quad \text { and } \quad \lambda(t) \equiv 0 \quad \text { on }|t| \geq \frac{2}{3} r_{0}
$$


where $r_{0}>0$ is sufficiently small. We define a distribution kernel on $W \times W$ by

$$
\begin{gathered}
T_{1}(x, y)=\lambda(|x-y|) E_{1}(x, y), \\
T_{1}^{\prime}(x, y)=\left(D_{1} \lambda(|x-y|)\right) E_{1}(x, y),
\end{gathered}
$$

and denote by $T_{1}$ and $T_{1}^{\prime}$ the linear operators from $C_{0}^{\infty}\left(W_{1}\right)$ to $C_{0}^{\infty}\left(W_{2}\right)$ whose distribution kernels are given by (4.3) and (4.4) respectively.

Lemma 4.1. $T_{1}^{\prime}$ can be written as a pseudodifferential type operator (see (4.16)) of the form

$$
T_{1}^{\prime} u(x)=\iint a\left(x, \xi^{1}\right) e^{i\langle x-y \mid \xi\rangle} u(y) d y \tilde{d} \xi,
$$

where $a\left(x, \xi^{1}\right)$ is a smooth function on $T^{*}\left(W_{2}\right)$ with the support of $a\left(x, \xi^{1}\right)$ in the $x$ variables contained in $W_{2}$, and satisfying, for any $N \in \mathbf{Z}_{+}, k \in \mathbf{Z}_{+}$and $\alpha$,

$$
\left|\left(\xi^{1}\right)^{N} \partial_{x}^{\alpha} \partial_{\xi^{\prime}}^{k} a\left(x, \xi^{1}\right)\right|<C_{N, k, \alpha} \quad \text { for any }(x, \xi) \in T \Omega_{2} .
$$

Proof. If we choose $r_{0}$ sufficiently small, we can write $T_{1}^{\prime} u(x)=\kappa(x) T_{1}^{\prime} u(x)$, for any $u \in C_{0}^{\infty}\left(W_{1}\right)$, where $\kappa(x)$ is a $C^{\infty}$ cutoff function on $\mathbf{R}^{n}$ such that $\kappa(x) \equiv 1$ on $\operatorname{dist}\left(x, W_{1}\right) \leq(2 / 3) r_{0}$, and $\equiv 0$ outside $W_{2}$. Then we have

$$
\begin{aligned}
T_{1}^{\prime} u(x) & =\iint \kappa(x) T_{1}^{\prime}(x, z) e^{i\langle z-y \mid \xi\rangle} u(y) d y d z \tilde{d} \xi \\
& =\int \hat{a} e^{-i\langle y \mid \xi\rangle} u(y) d y \tilde{d} \xi .
\end{aligned}
$$

Here we put

$$
\begin{aligned}
\hat{a} & =\int \kappa(x) T_{1}^{\prime}(x, z) e^{i\langle z \mid \xi\rangle} d z \\
& =\int \kappa(x)\left(D_{1} \lambda\left(\left|x^{1}-z^{1}\right|\right) H\left(x^{1}-z^{1}\right) e^{i\left\langle z^{1}-x^{1} \mid \xi^{1}\right\rangle} d z^{1} e^{i\langle x \mid \xi\rangle}\right. \\
& =a\left(x, \xi^{1}\right) e^{i\langle x \mid \xi\rangle}
\end{aligned}
$$

where $a\left(x, \xi^{1}\right)=-\int \kappa(x)\left(D_{1} \lambda(|t|)\right) H(t) e^{-i\left\langle t \mid \xi^{1}\right\rangle} d t$. Since this integrand is smooth and has compact support, we get (4.6).

Let $P$ be a pseudodifferential operator of order one defined on a relatively compact open set $U$. We may assume that $P$ is properly supported on $U$. Take an open set $V_{0}, 0 \in V_{0} \subset U$, sufficiently small so that the steps stated below hold. When we apply $P$ to functions defined on $U$, we shall use an appropriate coordinate system $\left(y^{1}, \ldots, y^{n}\right)$ on $U$. For emphasis, we occasionally denote this coordinate system by $U_{y}$. Let $p(y, \eta) \in S^{1}\left({ }^{o} T^{*} U_{y}\right)$ be the symbol function of $P$. We assume that the principal symbol $p_{1}(y, \eta) \in S_{0}^{1}\left({ }^{o} T^{*} U\right)$ of $p(y, \eta)$ satisfies $\left({ }^{*} 1\right)-\left({ }^{*} 3\right)$ for an open set $U_{y}$. Since we can apply the results 
stated in Proposition A.4 in Appendix A.II, we can take open conic finite coverings $\left\{\bar{D}_{J}\right\}$ and $\left\{D_{J}^{(0)}\right\}$ of ${ }^{o} T^{*} U$ and of ${ }^{o} T^{*} V_{0}$ respectively, (shrinking $V_{0}$ if necessary) such that

(i) for each $J, D_{J}^{(0)} \subset \bar{D}_{J}$ and $\operatorname{pr}\left(D_{J}^{(0)}\right)=V_{0}, \operatorname{pr}\left(\bar{D}_{J}\right)=U$;

(ii) $\cup D_{J}^{(0)}=\bigcup D_{j}^{(0)} \cup D_{*}^{(0)}$, where each $D_{j}^{(0)}$ contains a point $z_{j 0}=\left(0, \eta_{j 0}\right)$ satisfying $p_{1}\left(z_{j 0}\right)=0$ and in $D_{*}^{(0)}, p_{1}(z) \neq 0$;

(iii) there exists a canonical transformation $\chi: \bar{D}_{\left(0, e_{n}\right)} \rightarrow \bar{D}_{j}$ positively homogeneous of degree zero which satisfies the properties (i) in Proposition A.4; and

(iv) given $s_{4} \in \mathbf{Z}_{+}$, there exists $k_{4} \in \mathbf{Z}_{+}$and $\delta_{4}>0$, such that for $\left\|\tilde{p}_{1}-p_{1}\right\|_{k_{4}}<\delta_{4}$, and for each $j$, there exist a point $z_{j 0}\left(; \tilde{p}_{1}\right)=\left(0, \eta_{j 0}\left(\tilde{p}_{1}\right)\right)$ such that $\tilde{p}_{1}\left(z_{j 0}\left(; \tilde{p}_{1}\right)\right)=0$ and the properties (ii) in Proposition A.4 holds.

Remark. When we apply $P$ to $T_{j}$, etc., we use coordinates $\left(y^{1}, \ldots, y^{n}\right)$ chosen in (A.III). These coordinates depend only on $z_{0}$ and the principal symbol $p_{1}$, and not on any perturbation $\tilde{p}_{1}$ of $p_{1}$. Thus the norms for symbols are always computed with respect to these fixed coordinates.

Let $\left\{\phi_{J}\left(; p_{1}\right)\right\}$ be the partition of unity positively homogeneous of degree zero associated with the coverings $\left\{D_{J}^{(0)}\right\}$ and $n_{0}=[n / 2]+1$. Take sequences of open sets $0 \in W_{1} \subset W_{2} \subset W$ and $0 \in V_{0} \subset V_{1} \subset V_{2} \subset U$ appropriately small and also choose sequences of open conic sets $D_{\left(0, e_{n}\right)}^{(1)} \subset D_{\left(0, e_{n}\right)}^{(2)} \subset \bar{D}_{\left(0, e_{n}\right)}$ and $D_{j}^{(0)} \subset D_{j}^{(1)} \subset D_{j}^{(2)} \subset \bar{D}_{j}$ such that $\operatorname{pr}\left(D_{\left(0, e_{n}\right)}^{(i)}\right)=W_{i},(i=1,2)$ and $\operatorname{pr}\left(D_{j}^{(i)}\right)=$ $V_{i},(i=0,1,2)$. Let $I$ be the identity operator on $C^{\infty}\left(V_{0}\right)$. Associated with $\left\{D_{J}^{(0)}\right\}$ and $\left\{\phi_{J}(y, \eta)\right\}$, we divide $I$ microlocally into $I=\sum I_{J}$, where

$$
\left(I_{J} u\right)\left(y^{\prime}\right)=\iint \phi_{J}\left(y^{\prime}, y, \eta\right) e^{i\left\langle y^{\prime}-h \mid \eta\right\rangle} u(y) d y d \eta .
$$

Here, we put $\phi_{J}\left(y^{\prime}, y, \eta\right)=\phi\left(y^{\prime}\right) \phi_{J}(y, \eta)$, where $\phi\left(y^{\prime}\right)$ is a cutoff function, $\phi\left(y^{\prime}\right) \equiv 1$, on $V_{0}$, and $\operatorname{supp} \phi \subset V_{1}$. Take $r_{0}$ in (4.2) and $V_{0}$ sufficiently small, and choose linear operators $T_{1}, T_{1}^{\prime}: C_{0}^{\infty}\left(W_{1}\right) \rightarrow C_{0}^{\infty}\left(W_{2}\right), F_{j}^{+}: C_{0}^{\infty}\left(V_{1}\right) \rightarrow$ $C_{0}^{\infty}\left(W_{1}\right)$ and $F_{j}: C_{0}^{\infty}\left(W_{2}\right) \rightarrow C_{0}^{\infty}\left(V_{2}\right)$ which satisfy Theorem A.10 and have the following properties:

$$
\begin{cases}W F\left(I_{j} u\right) \subset D_{j}^{(1)} & \text { for } W F(u) \subset D_{j}^{(0)}, \\ W F\left(F_{j}^{+} f\right) \subset D_{\left(0, e_{n}\right)}^{(1)} & \text { for } W F(f) \subset D_{\left(0, e_{n}\right)}^{(0)}, \\ W F\left(T_{1} v\right) \subset D_{\left(0, e_{n}\right)}^{(2)} & \text { for } W F(v) \subset D_{\left(0, e_{n}\right)}^{(1)} \\ W F\left(T_{1}^{\prime} v\right) \subset D_{\left(0, e_{n}\right)}^{(2)} & \text { for } W F(v) \subset D_{\left(0, e_{n}\right)}^{(1)}, \\ W F\left(F_{j} g\right) \subset D_{j}^{(2)} & \text { for } W F(g) \subset D_{\left(0, e_{n}\right)}^{(2)} .\end{cases}
$$

Set $T_{j}=F_{j} T_{1} F_{j}^{+} I_{j}$. We get

$$
P T_{j}=P F_{j} T_{1} F_{j}^{+} I_{j}=\left(P F_{j}-F_{j} D_{1}\right) T_{1} F_{j}^{+} I_{j}+F_{j} D_{1} T_{1} F_{j}^{+} I_{j} .
$$


By considering the wave front set of $T_{1} F_{j}^{+} I_{j} f$, for $f \in D^{\prime}\left(U_{1}\right)$, we easily see that

$$
K_{j,-\infty}^{\prime}=\left(P F_{j}-F_{j} D_{1}\right)
$$

can be viewed as a Fourier integral operator whose symbol is of order $-n_{0}$ if we choose the open set $V_{0}$ and the support of $\lambda(t)$ small enough $[\mathrm{H}, \mathrm{p}$. 71] and $D_{\left(0, e_{n}\right)}^{(2)}$ is chosen to be contained in $D_{\left(0, e_{n}\right)}$ given in Theorem A.10, Appendix (A.III). Moreover, we can fix $\lambda(t)$ for any $\tilde{p}$ sufficiently close to $p$. Now, we consider the second term of the right-hand side of $(4.10)$. We write $D_{1} T_{1}=I+T_{1}^{\prime}$ and $T_{1}^{\prime}=T_{1}^{\prime \prime}+T_{1,-\infty}^{\prime}$, where for any $u \in C_{0}^{\infty}\left(W_{1}\right)$,

$$
\begin{aligned}
T_{1}^{\prime \prime} u(x) & =\iint a^{\prime}\left(x, \xi^{1}\right) e^{i\left\langle x-x^{\prime} \mid \xi\right\rangle} u\left(x^{\prime}\right) d x^{\prime} d \xi, \\
T_{1,-\infty}^{\prime} u(x) & =\iint a_{-\infty}\left(x, \xi^{1}\right) e^{i\left\langle x-x^{\prime} \mid \xi\right\rangle} u\left(x^{\prime}\right) d x^{\prime} d \xi .
\end{aligned}
$$

Here we put $a^{\prime}\left(x, \xi^{1}\right)=\left(1-\nu\left(\xi^{1}\right)\right) a\left(x, \xi^{1}\right), a_{-\infty}\left(x, \xi^{1}\right)=\nu\left(\xi^{1}\right) a\left(x, \xi^{1}\right)$ and $\nu\left(\xi^{1}\right)$ satisfies $\nu \equiv 1$ on $\left|\xi^{1}\right| \leq r_{0}^{\prime} / 2$, and $\nu\left(\xi^{1}\right) \equiv 0$ on $\left|\xi^{1}\right| \geq r_{0}^{\prime}$ for some constant $r_{0}^{\prime}>0$. Since

$$
K_{j,-\infty}^{\prime \prime}=F_{j} F_{j}^{+} I_{j}-I_{j}=\left(F_{j} F_{j}^{+}-I\right) I_{j}
$$

can be viewed as a pseudodifferential operator of order $-n_{0}$ by Theorem A.10, we can write $(4.10)$ as

$$
P T_{j}=I_{j}+K_{j,-\infty}+R_{j}
$$

where

and

$$
K_{j,-\infty}=K_{j,-\infty}^{\prime} T_{1} F_{j}^{+} I_{j}+K_{j-\infty}^{\prime \prime}
$$

$$
R_{j}(; p)=F_{j} T_{1}^{\prime \prime} F_{j}^{+} I_{j}+F_{j} T_{1,-\infty}^{\prime} F_{j}^{+} I_{j}
$$

Now, we consider the following symbol class for $j \in \mathbf{Z}$ : for any $N \in \mathbf{Z}_{+}$, $k \in \mathbf{R}$ and $\alpha, \beta, \gamma$,

$$
\begin{aligned}
& \left\|g\left(x^{\prime}, x, \xi\right) \mid\right\|_{j, N, m} \\
& \quad=\sup _{|\alpha|+|\beta|+|\gamma| \leq m}\left\|\left(1+\left|\xi^{1}\right|\right)^{N}(1+|\xi|)^{|\beta|-j} \partial_{x^{\prime}}^{\alpha} \partial_{\xi 1}^{k} \partial_{\xi}^{\beta} \partial_{x}^{\gamma} g_{j}\right\| \leq C_{\alpha, \beta, \gamma, k, N},
\end{aligned}
$$

for $\left(x^{\prime}, x, \xi\right) \in \bar{D}_{\left(0, e_{n}\right)}$, where we put $\xi=\left(\xi^{1}, \tilde{\xi}\right), \tilde{\xi}=\left(\xi^{2}, \ldots, \xi^{n}\right)$. Associated to the symbol $g\left(x^{\prime}, x, \xi\right)$, is a linear operator $G$ defined by

$$
G u\left(x^{\prime}\right)=\iint g\left(x^{\prime}, x, \xi\right) e^{i\langle y-x \mid \xi\rangle} u(x) d x d \xi,
$$

which is called a pseudodifferential type operator of order $j$.

Now, we seek $\bar{G}$ such that

$$
D_{1} \bar{G}=T_{1}^{\prime \prime}
$$

as an operator from $C_{0}^{\infty}\left(W_{1}\right)$ to $C^{\infty}\left(W_{2}\right)$. 
Now, (4.17) can be solved by the following partial differential equation

$$
\partial_{x^{1}} \bar{g}+i \xi^{1} \bar{g}=a^{\prime}\left(x, \xi^{1}\right)
$$

The solution can be found explicitly as

$$
\bar{g}\left(x, \xi^{1}\right)=\kappa^{\prime}(x) \int_{0}^{x^{1}} a^{\prime}\left(x, \xi^{1}\right) e^{-i\left\langle x^{1}-t \mid \xi^{1}\right\rangle} d t
$$

where $\kappa^{\prime}(x)$ is a $C^{\infty}$ cutoff function $\kappa(x) \equiv 1$ on $\operatorname{dist}\left(x, W_{2}\right)<r^{\prime \prime} / 2$, and $\kappa(x) \equiv 0$ outside $W$, for some positive $r^{\prime \prime} / 2$, because the support of $a^{\prime}$ in the $x$ variables is contained in $W$. Combining (4.12) and (4.13) with the above and arguing as in (4.14), we get on $U_{1}$,

$$
\begin{aligned}
P F_{j} \bar{G} F_{j}^{+} I_{j} & =K_{j,-\infty}^{\prime} \bar{G} F_{j}^{+} I_{j}+F_{j} D_{1} \bar{G} F_{j}^{+} I_{j} \\
& =R_{j}+R_{j,-\infty}^{(1)}+R_{j,-\infty}^{(2)},
\end{aligned}
$$

where

$$
R_{j,-\infty}^{(1)}=K_{j,-\infty}^{\prime} \bar{G} F_{j}^{+} I_{j}, \quad R_{j,-\infty}^{(2)}=F_{j} T_{1,-\infty}^{\prime} F_{j}^{+} I_{j} .
$$

Here $K_{j,-\infty}^{\prime}$ can be viewed as a Fourier integral operator of order $-n_{0}$, if we choose $W F(\bar{G} g) \subset D_{\left(0, e_{n}\right)}$ for $W F(g) \subset D_{\left(0, e_{n}\right)}^{(2)}, \bar{G}$ is a pseudodifferential type operator order zero with the symbol $\bar{g}$, and $T_{1, \infty}^{\prime}$ is defined by (4.13) and is also a pseudodifferential type operator of order $-n_{0}$. On $D_{*}^{(0)}$, we can easily construct an elliptic pseudodifferential operator $T_{*}$ satisfying $P T_{*}=I+R_{*,-\infty}$ on $D_{*}^{(0)}$ where $R_{*,-\infty}$ is order $-n_{0}$; the behavior of $T_{*}$ under perturbation of $p$ can be easily seen. Thus, we get the following:

Theorem 4.2. In the above situations, we get the following:

(i) There exists a right parametrix $E(; p): C_{0}^{\infty}\left(V_{0}\right) \rightarrow C^{\infty}\left(V_{0}\right)$ of $P$ defined on an open $U_{1}$ in the following form

$$
P E(; p)=I+L_{-\infty}(; p),
$$

where

$$
L_{-\infty}=\sum_{j} K_{j,-\infty}-R_{j,-\infty}^{(1)}-R_{j,-\infty}^{(1)}+R_{*,-\infty}
$$

and

$$
E(; p)=\sum_{j} F_{j}(; p) T_{1} F_{j}^{+}(; p) I_{j}-F_{j}(; p) \bar{G} F_{j}^{+}(; p) I_{j}+T_{*}
$$

where $F_{j}(; p), F_{j}^{+}(; p)$ are Fourier integral operators with the symbol $a(; p)=$ $a_{j}(x, \eta, y ; p)$ and $b=b_{j}(y, \xi, x ; p)$ and the phase function $S(x, \eta ; \chi)$ satisfies Theorem A.10. Furthermore, $K_{j,-\infty}(; p)=K_{j,-\infty}^{\prime}(; p)+K_{j,-\infty}^{\prime \prime}(; p)$ is defined by (4.11) and (4.14), $K_{j,-\infty}^{\prime}$ can be viewed as a Fourier integral operator of order $-n_{0}$ with the symbol function $k_{j}(; p), R_{j,-\infty}^{(i)}, i=1,2$, are defined by 
(4.23), and $\bar{G}(; p)$ is defined as above with symbol function $\bar{g}(; p)$ satisfying the estimate (4.16).

(ii) Moreover, there exist $k_{12} \in \mathbf{Z}_{+}$, and $\delta_{12}>0$ such that for any $m_{12} \in \mathbf{Z}_{+}$, the following properties hold: for any $\|\tilde{p}-p\|_{1, k_{12}}<\delta_{12}$, we can construct a right parametrix $E(; \tilde{p})$ as in (4.21) and (4.22) and we have

$$
\left\|k_{j}(; \tilde{p})-k_{j}(; p)\right\|_{0, m_{12}} \leq C_{12}\|\tilde{p}-p\|_{1, m_{12}+k_{12}}
$$

for some positive constant $C_{12}$.

To get the tame estimate (3.2), we first remark that for $F(a, \chi)$ given as above,

$$
\|F(\tilde{a}, \tilde{\chi}) f\|_{s} \leq C\left\{|\tilde{\chi}|_{s_{0}+s}+\|\tilde{a}\|_{s_{0}+s}+\sum_{j}|\chi|_{s_{0}+s-j}+\|\tilde{a}\|_{s_{0}+s-j}\|f\|_{s_{0}+j}\right\},
$$

which is a direct computation as in $\S 5$, Lemma 5.7 combined with the $L^{2}$ boundedness of Fourier integral operators (cf. [K]). Now, the estimate for $\bar{G}$ can be reduced to an estimate for a usual classical pseudodifferential operator on $\mathbf{R}^{n-1}$ depending on a parameter by the following argument. Set $\tilde{g}\left(x^{1}, y^{1}, x^{\prime}, \xi^{\prime}\right)=\int e^{i\left\langle x^{1}-y^{1} \mid \xi^{1}\right\rangle} \bar{g}\left(x^{1}, x^{\prime}, \xi^{1}, \xi^{\prime}\right) d \xi^{1}$. Then, for any $k \in \mathbf{Z}_{+}$, $\alpha, \beta \in \mathbf{Z}_{+}^{n-1}$, we clearly have

$$
\sup \left(1+\left|x^{1}-y^{1}\right|\right)^{k}\left|\partial_{x^{1}}^{\alpha} \partial_{\xi^{1}}^{\beta} \tilde{g}\left(x^{1}, y^{1}, x^{\prime}, \xi^{\prime}\right)\right|\left(1+\left|\xi^{\prime}\right|\right)^{|\beta|}<\infty,
$$

where the supremum is taken over $x^{1}, y^{1} \in \mathbf{R}, x^{\prime}, y^{\prime} \in \mathbf{R}^{n-1}$. Hence, if we set $\widetilde{G}\left(x^{1}, y^{1}\right)=\tilde{g}\left(x^{1}, y^{1}, x^{\prime}, D_{x^{\prime}}\right)$, there exists $s_{0} \in \mathbf{Z}_{+}$such that, for any $\alpha \in \mathbf{Z}_{+}^{n}$,

$$
\left\|\partial_{x}^{\alpha}\left(\widetilde{G}\left(x^{1}, y^{1}\right) v\right)\left(x^{\prime}\right)\right\|_{L^{2}\left(\mathbf{R}_{x^{\prime}}^{n-1}\right)} \leq c\left(1+\left|x^{1}-y^{1}\right|\right)^{-1} \sum_{\nu=0}^{|\alpha|}\|\bar{g}\|_{s_{0}+\nu}\|v\|_{|\alpha|-\nu}
$$

with some constant $C$ depending only on $\alpha$. Therefore, by Minkowski's inequality, we have

$$
\left\|\partial_{x}^{\alpha}(\bar{G} u)\right\|_{0} \leq C \sum_{\nu=0}^{|\alpha|}\|\bar{g}\|_{s_{0}+\nu}\|u\|_{|\alpha|-\nu}
$$

with some other constant $C$ depending only on $\alpha$.

Now, we let $\Phi^{\prime}(u)$ be the partial differential operator of Theorem 3.1. By applying the argument of [DH, pp. 199-200] to $M(x, D ; u)$ given by (3.6), we can replace it by $l(x, D ; u)\left(\bmod S^{-n_{0}}\right)$. Define the pseudodifferential operator $P(u)$ of order one to be $l(x, D ; u)$ times an appropriate elliptic operator. There exists $m_{0} \in \mathbf{Z}_{+}$, such that for any $k \in \mathbf{Z}_{+}$,

$$
\|p(\tilde{u})-p(u)\|_{1, k} \leq C\|\tilde{u}-u\|_{k+m_{0}} .
$$


As in the argument after Lemma 5.5, we can drop $L_{-\infty}$ in (4.21) and the appropriate tame estimate for this procedure can be easily seen by Lemma 5.6. Then, combining (4.25)-(4.28), we get the estimate (3.2).

\section{Proof of Theorem E}

The purpose of this section is to present a proof of Theorem $\mathrm{E}$ which seems to be the simplest and shortest proof.

Let $x_{0}, U_{0}, u_{0}, \Phi(u) \equiv \Phi\left(x, \bar{D}^{m} u\right)$ be the same as those in the beginning of $\S 3$, and let $L(u), l(x, D ; u), M(x, D ; u)=l(x, D ; u) I+R(x, D ; u)$ be the same as those defined by (3.4), (3.5) and (3.6) respectively. Since we are seeking a local right inverse of $M(x, D ; u)$, we may assume $M(x, D ; u) \equiv 0$ outside $|x| \leq R$.

Hereafter, we frequently use the abbreviated notation such as $\Phi(u)=$ $\Phi\left(x, \bar{D}^{m} u\right)$ and quote the results in Chapter 10 (mostly $\S 4$ ) of [K]. For the convenience of readers references, we underline all the numbers attached to the equations, theorems and others in $[\mathrm{K}]$ whenever we quote them in this section.

Set $\lambda\left(x, D_{x} ; u\right)=\lambda\left(s, D_{x} ; u\right) \mathbf{I}$ and $\lambda+\mathbf{B}^{\prime}=M(x, D ; u)\left\langle D_{x}\right\rangle^{-m N+1}$, where $I$ denotes $N \times N$ identity operator, $\lambda(x, \xi ; u)=(1-\chi(\xi))|\xi|^{-m N+1} l(x, \xi ; u) \in S^{1}$, $\chi \in C_{0}^{\infty}\left(\mathbf{R}^{n}\right), 0 \leq \chi \leq 1, \chi(\xi)=1(|\xi| \leq 1)$ and $\mathbf{B}^{\prime} \in S^{0}$. Here a $C^{\infty}$ map $u: \mathbf{R}^{n} \rightarrow \mathbf{R}^{N}$ is sufficiently close to $u_{0}$ with respect to some Sobolev norm, which is expressed by $u \sim u_{0}$ in the mean while.

In order to construct a local right inverse of $\lambda+\mathbf{B}^{\prime}$ with the so-called tamed estimate, we consider the following initial value problem:

$$
\left\{\begin{array}{l}
\frac{(4.19 \mathrm{i})}{(4.19 \mathrm{ii})} \quad \mathbf{M} U=0 \quad \text { on }\left[0, T_{0}\right], \\
U_{\mid t=0}=G \in H_{-\infty} .
\end{array}\right.
$$

Before going into the details, we explain the outline of the proof.

According to Theorem 4.5, there exists an approximate fundamental solution $\widetilde{E}_{\phi}(t)=\tilde{\mathbf{e}}_{\phi}\left(t, x, D_{x} ; u\right) \in B_{t}^{1}\left(S^{0}\right)$ of $\underline{(4.19)}$ on $\left[0, T_{0}\right]$. Namely,

$$
\begin{cases}\underline{(4.21 \mathrm{i})} & \mathbf{M} \widetilde{\mathbf{E}}_{\phi}(t ; u)=\widetilde{\mathbf{R}}_{\phi}(t, u) \in B\left(S^{-(n+1)}\right) \quad \text { on }\left[0, T_{0}\right], \\ \underline{(4.21 \mathrm{ii})} & \widetilde{\mathbf{E}}_{\phi}(0 ; u)=I .\end{cases}
$$

Moreover, from Theorem 3.14, p. $\underline{308}$

$$
\begin{aligned}
W F\left(\widetilde{\mathbf{E}}_{\phi}(t ; u) G\right) \subset\{(x, \xi)=\tilde{T}(t ; u)(y, \eta) ;(y, \eta) \in W F(G)\} & W \quad \text { for any } G \in H_{-\infty},
\end{aligned}
$$

where

$$
\widetilde{T}(t ; u)(y, \eta)=(q(t, y, \eta ; u), p(t, y, \eta ; u))
$$

and $q(t, y, \eta ; u), p(t, y, \eta ; u)$ are the solutions of the initial value problem:

$$
\frac{d q}{d t}=\nabla_{\xi} \lambda(q, p ; u), \quad \frac{d p}{d t}=-\nabla_{x} \lambda(q, p ; u), \quad(q, \dot{p})_{\mid t=0}=(y, \eta) .
$$

Since $\lambda\left(u_{0}\right)+\mathbf{B}^{\prime}\left(u_{0}\right)$ is real principal type at $x_{0}$ and this condition is an open condition, $\lambda+\mathbf{B}^{\prime}(u)$ is also real principal type at $x_{0}$ for any $u\left(u \sim u_{0}\right)$. 
Thus, there exist an open neighborhood $U_{2} \subset U$ of $x_{0}$ and $T_{1}\left(0<T_{1}<T_{0}\right)$ such that

$$
\widetilde{T}\left(T_{1} ; u\right)\left({ }^{o} T^{*} U_{2}\right) \cap{ }^{o} T^{*} U_{2}=\phi .
$$

Now, take $\psi(t) \in C_{0}^{\infty}\left(\left[0, T_{0}\right]\right)$ such that $\psi(t)=1 \quad\left(0 \leq t \leq T_{1}\right)$ and set

$$
\mathbf{Q} G=i \int_{0}^{\infty} \psi(t) \widetilde{\mathbf{E}}_{\phi}(t ; u) G d t \quad\left(G \in H_{-\infty}\right) .
$$

Then, by $\underline{(4.21)}$ and integration by parts,

$$
\begin{aligned}
\left(\lambda+\mathbf{B}^{\prime}\right) \mathbf{Q} G & =i \int_{0}^{\infty} \psi(t)\left(\lambda+\mathbf{B}^{\prime}\right) \widetilde{\mathbf{E}}_{\phi}(t) G d t \\
& =-i \int_{0}^{\infty} \psi(t)\left(D_{t} \widetilde{\mathbf{E}}_{\phi}(t) G-\widetilde{\mathbf{R}}_{\phi}(t) G\right) d t \\
& =G+\int_{0}^{\infty} \psi^{\prime}(t) \widetilde{\mathbf{E}}_{\phi}(t) G d t-i \int_{0}^{\infty} \psi(t) \widetilde{\mathbf{R}}_{\phi}(t) G d t .
\end{aligned}
$$

Here, $-\left(\int_{0}^{\infty} \psi^{\prime}(t) \widetilde{\mathbf{E}}_{\phi}(t) d t-i \int_{0}^{\infty} \psi(t) \widetilde{\mathbf{R}}_{\phi}(t) G d t\right)$ has a unique distribution kernel $\widetilde{\mathbf{K}} \in L_{\text {loc }}^{1}\left(U_{2} \times U_{2}\right)$, because of $\underline{(4.21)},(5.1)$ and (5.3). Then, we shall prove that $\mathbf{Q}, \mathbf{K}=\phi_{2} \tilde{\mathbf{K}} \phi_{3}, \lambda+\mathbf{B}^{\prime}$ correspond to $Q(u), K(u), M(u)$ of Lemma 3.3 and satisfy (3.9)-(3.14) for appropriate cut off functions $\phi_{1}, \phi_{2}, \phi_{3}$. Hence, $\mathbf{Q}(u)(1-\mathbf{K}(u))^{-1} \phi_{2}$ is a local right inverse of $\lambda(u)+\mathbf{B}^{\prime}(u)$ with the so-called tamed estimate. Of course, the key lemma of the proof is to prove the estimate (3.9), (3.14) in Lemma 3.3 and the rest of the proof is quite formal.

Lemma 5.1. Let $n_{0}=[n / 2]+1 \quad\left(n_{0}\right.$ stands for this particular constant for the rest of this paper). For any $w_{0}>0, \alpha, \beta \in \mathbf{Z}_{+}^{n}$, there exists a constant $c=$ $c\left(\alpha, \beta, w_{0}\right)>0$ such that

$$
\begin{aligned}
\sup _{\xi}\left\|\partial_{x}^{\alpha} \partial_{\xi}^{\beta} \lambda\left(x, \xi ; \bar{D}^{m} u\right)\langle\xi\rangle^{|\beta|-1}\right\|_{n_{0}} & \leq C\left(1+\|u\|_{|\alpha|+m+n_{0}}\right), \\
\sup _{\xi}\left\|\partial_{x}^{\alpha} \partial_{\xi}^{\beta} \mathbf{B}_{j}^{\prime}\left(x, \xi ; \bar{D}^{m+j+1} u\right)\langle\xi\rangle^{j+|\beta|}\right\|_{n_{0}} & \leq C\left(1+\|u\|_{|\alpha|+m+j+n_{0}+1}\right), \\
(u & \left.\in B^{\infty}\left(\mathbf{R}^{n}\right),\left\|\bar{D}^{m N} u\right\|_{L^{\infty}} \leq w_{0}\right)
\end{aligned}
$$

where

$$
\mathbf{B}^{\prime}\left(x, \xi ; \bar{D}^{m} u\right)=\sum_{j=0}^{m N-m-1} \mathbf{B}_{j}^{\prime}\left(x, \xi ; \bar{D}^{m+j+1} u\right) .
$$

This can be easily proved by the chain rule for differentiating composite functions and the following well known property of the Sobolev norm $\|v\|_{s}$ :

$$
\|v\|_{s} \leq c\|v\|_{s_{1}}^{\theta_{1}}\|v\|_{s_{2}}^{\theta_{2}}
$$

where the constant depends only on $s, s_{1}, s_{2}\left(s_{1} \leq s \leq s_{2}\right)$, and $0 \leq \theta_{1}, \theta_{2} \leq 1$ is defined by $\theta_{1} s_{1}+\theta_{2} s_{2}=s, \theta_{1}+\theta_{k 2}=1$.

From Theorem 4.1 and its proof, we have the following lemma. 
Lemma 5.2. For any $w_{0}>0,0<\tau<1$, there exists $0<T<T_{0}$ such that, for any $u \in B^{\infty}\left(\mathbf{R}^{n}\right)$ satisfying $\|u\|_{m+n_{0}+2} \leq w_{0}$, the eikonal equation

$$
\begin{array}{ccc}
\frac{(4.20 \mathrm{i})}{(4.20 \mathrm{ii})} & \phi_{t}(t, x, \xi ; u)+\lambda\left(x, \nabla_{x} \phi(t, x, \xi ; u) ; u\right)=0 & \text { on }[0, T] \\
\phi(0, x, \xi ; u)=\langle x \mid \xi\rangle &
\end{array}
$$

admits a unique solution $\phi(t, x, \xi ; u) \in \mathscr{P}(\tau)$ which satisfies

$$
\begin{gathered}
J(t, x, \xi ; u)=\phi(t, x, \xi ; u)-\langle x \mid \xi\rangle \in S_{1}^{1}((2)), \\
\sum_{|\alpha+\beta| \leq 2} \sup _{x, \xi} \| \partial_{x}^{\alpha} \partial_{\xi}^{\beta} J(t, x, \xi ; u) \mid\langle\xi\rangle^{|\beta|-1} \leq \tau
\end{gathered}
$$

and

$$
\sum_{|\alpha+\beta| \leq 2} \sup _{\xi}\left\|\partial_{x}^{\alpha} \partial_{\xi}^{\beta} J(t, x, \xi ; u)\right\|_{n_{0}}\langle\xi\rangle^{|\beta|-1}<\infty
$$

for $0 \leq t \leq T,\|u\|_{m+n_{0}+2} \leq w_{0}$.

Next we proceed to estimate the higher order derivatives of $J(t, x, \xi ; u)$ and the symbol $\tilde{\mathbf{e}}(t, x, \xi ; u)$ of $\widetilde{\mathbf{E}}_{\phi}(t)$ defined by

$$
\tilde{\mathbf{e}}(t, x, \xi ; u)=\sum_{\nu=0}^{n} \mathbf{e}_{\nu}(t, x, \xi ; u),
$$

$\underline{(4.49 \mathrm{ii})}$

$$
\mathbf{e}_{\nu}(t, x, \xi ; u) \in \mathbf{B}_{t}^{1}\left(S^{-\nu}\right) \text { on }[0, T]
$$

$$
\underline{\left(4.64 \mathrm{i}_{0}\right)} \quad D_{t}\left(\mathbf{e}_{0}-I\right)+\sum_{j=1}^{n} a_{j}(t) D_{x^{j}}\left(\mathbf{e}_{0}-I\right)
$$

$\underline{(4.64)}$

$$
\begin{array}{lll} 
& \multicolumn{1}{c}{+\mathbf{H}(t)\left(e_{0}-I\right)+\mathbf{H}(t)=0 \quad \text { on }[0, T],} \\
\frac{\left(4.64 \mathrm{ii}_{0}\right)}{\left(4.64 \mathrm{i}_{\nu}\right)} & \mathbf{e}_{0}(0)-I=0, \\
\frac{D_{t} \mathbf{e}_{\nu}+\sum_{j=1}^{n} a_{j}(t) D_{x^{j}} \mathbf{e}_{\nu}+\mathbf{H}(t) \mathbf{e}_{\nu}+\mathbf{R}_{\nu-1}(t) \quad \text { on }[0, T],}{\left(4.64 \mathrm{ii}_{\nu}\right)} & \mathbf{e}_{\nu}(0)=0 \quad(\nu \geq 1),
\end{array}
$$

where

$$
a_{j}(t)=\lambda^{(j)}\left(x, \nabla_{x} \phi(t, x, \xi ; u) ; u\right)
$$

$\underline{(4.60)}$

$$
\begin{aligned}
\mathbf{H}(t)= & -\frac{i}{2}\left\{\sum_{j, k=1}^{n} \lambda^{(j, k)}\left(x, \nabla_{x} \phi(t, x, \xi ; u) ; u\right) \frac{\partial^{2}}{\partial x^{j} \partial x^{k}} \phi(t, x, \xi ; u)\right\} I \\
& +\mathbf{B}_{0}^{\prime}\left(x, \nabla_{x} \phi(t, x, \xi ; u) ; u\right) \\
\mathbf{R}_{\nu-1}(t, x, \xi ; u) & \\
= & \sum_{1} \frac{1}{\alpha !} D_{x^{\prime}}^{\alpha}\left\{\lambda^{(\alpha)}\left(x, \widetilde{\nabla}_{x} \phi\left(t, x, x^{\prime}, \xi ; u\right) ; u\right) \mathbf{e}_{\mu}\left(t, x^{\prime}, \xi ; u\right)\right\}_{\mid x^{\prime}=x} \\
& +\sum_{2} \frac{1}{\alpha !} D_{x^{\prime}}^{\alpha}\left\{\mathbf{B}_{j}^{(\alpha)}\left(x, \tilde{\nabla}_{x} \phi\left(t, x, x^{\prime}, \xi ; u\right) ; u\right) \mathbf{e}_{\mu}\left(t, x^{\prime}, \xi ; u\right)\right\}_{\mid x^{\prime}=x}
\end{aligned}
$$


with the summations $\sum_{1}$ and $\sum_{2}$ taken for $\alpha, \mu(|\alpha| \geq 2,|\alpha|+\mu=1+\nu)$ and $\alpha, \mu, j \quad(|\alpha| \geq 1,|\alpha|+j+\mu=\nu)$, and

$$
\tilde{\nabla}_{x} \phi\left(t, x, x^{\prime}, \xi ; u\right)=\int_{0}^{1} \nabla_{x} \phi\left(t, x^{\prime}+\theta\left(x-x^{\prime}\right), \xi ; u\right) d \theta .
$$

Note that, for each $3 \leq m \in \mathbf{Z}_{+}$, the derivatives $\partial_{x}^{\alpha} \partial_{\xi}^{\beta} J\left(\alpha, \beta \in \mathbf{Z}_{+}^{n}, \mid \alpha+\right.$ $\beta \mid=m$ ) satisfy a symmetric hyperbolic system quite similar to (4.64). More precisely,

$$
D_{t} \partial_{x}^{\alpha} \partial_{\xi}^{\beta} J+\sum_{j=1}^{n} a_{j}(t) D_{x^{j}} \partial_{x}^{\alpha} J+R_{\alpha, \beta}=0 \quad \text { on }[0, T], \quad \partial_{x}^{\alpha} \partial_{\xi}^{\beta} J_{\mid t=0}=0
$$

where $R_{\alpha, \beta}$ is determined by the part of derivative $\partial_{x}^{\alpha} \partial_{\xi}^{\beta} \lambda\left(x, \nabla_{x} \phi ; \bar{D}^{m} u\right)$ and it contains the derivatives of $J$ with respect to $(x, \xi)$ up to order $m-1$. The following lemma is useful for the estimates of $\partial_{x}^{\alpha} \partial_{\xi}^{\beta} J\left(\alpha, \beta \in \mathbf{Z}_{+}^{n},|\alpha+\beta|=m\right)$ and $\mathbf{e}_{\nu}(0 \leq \nu \leq n)$.

Lemma 5.3 (cf. [M, p. 312, Proposition 6.1]). Fix $s \in \mathbf{Z}_{+}$and let $\mathbf{A}_{k}(t, x)$ $(1 \leq k \leq n), \mathbf{B}(t, x)$ are $N \times N$ matrices. Assume $\mathbf{A}_{k}(t, x)(1 \leq k \leq n)$ are Hermitian and $\mathbf{A}_{k}(t, x) \in B_{t}^{0}\left(B^{\max (1, s)}\left(\mathbf{R}_{x}^{n}\right)\right)(1 \leq k \leq n), \quad \mathbf{B}(t, x) \in$ $B_{t}^{0}\left(B^{s}\left(\mathbf{R}_{x}^{n}\right)\right)$ on $[0, T]$. If $v(t, x) \in B_{t}^{1}\left(H^{s}\left(\mathbf{R}_{x}^{n}\right)\right)$ on $[0, T]$ satisfies the symmetric hyperbolic system:

$$
D_{t} v-\sum_{k=1}^{n} \mathbf{A}_{k}(t, x) D_{x^{k}} v-\mathbf{B}(t, x) v=f(t, x) \quad \text { on }[0, T]
$$

for a given vector function $f(t, x) \in B_{t}^{0}\left(H^{s}\left(\mathbf{R}_{x}^{n}\right)\right)$ on $[0, T]$, then there exists a constant depending only on $s, T$,

$$
\sum_{|\alpha| \leq \max (1, s)} \sup _{t}\left\|\partial_{x}^{\alpha} \mathbf{A}_{k}(t, \cdot)\right\|_{L^{\infty}}, \quad \sum_{|\alpha| \leq s} \sup _{t}\left\|\partial_{x}^{\alpha} \mathbf{B}_{k}(t, \cdot)\right\|_{L^{\infty}},
$$

such that

$$
\|v(t, \cdot)\|_{s} \leq C\left(\|v(0, \cdot)\|_{s}+\int_{0}^{t}\|f(\tau, \cdot)\|_{s} d \tau\right) \quad(0 \leq t \leq T) .
$$

Apply $\partial_{x}^{\alpha} \partial_{\xi}^{\beta}$ to $(4.64 \mathrm{i}),\left(4.64 \mathrm{i}_{\nu}\right)$ and argue inductively on $|\alpha+\beta|$ and $\nu$ with the aid of (5.8) and Lemma 5.3 for $s=n_{0}$, we have the following lemma for the estimates for $\partial_{x}^{\alpha} \partial_{\xi}^{\beta} J$ and $\partial_{x}^{\alpha} \partial_{\xi}^{\beta} \mathbf{e}_{\nu}$.

Lemma 5.4. Let $w_{0}, T$ be the same as those in Lemma 5.2. Then, for any $\alpha$, $\beta \in \mathbf{Z}_{+}^{n}, 0 \leq \nu \leq n$, there exists a constant $C$ depending only on $w_{0}, \alpha, \beta$, $n_{0}$ such that

$$
\sup _{\xi}\left\|\partial_{x}^{\alpha} \partial_{\xi}^{\beta} J(t, x, \xi ; u)\right\|_{n_{0}}\langle\xi\rangle^{|\beta|-1} \leq C t\left(1+\|u\|_{m+|\alpha|+n_{0}}\right),
$$




$$
\begin{aligned}
& \sup _{\xi}\left\|\partial_{x}^{\alpha} \partial_{\xi}^{\beta}\left(\mathbf{e}_{0}-I\right)\right\|_{n_{0}}\langle\xi\rangle^{|\beta|} \leq C\left(1+\|u\|_{m+|\alpha|+n_{0}+2}\right), \\
& \sup _{\xi}\left\|\partial_{x}^{\alpha} \partial_{\xi}^{\beta} \mathbf{e}_{\nu}\right\|_{n_{0}}\langle\xi\rangle^{|\beta|+\nu} \leq C\left(1+\|u\|_{m+|\alpha|+n_{0}+2(\nu+1)}\right),
\end{aligned}
$$

for $0 \leq t \leq T, u \in B^{\infty}\left(\mathbf{R}^{n}\right) \quad\left(\|u\|_{m N+n_{0}}+\|u\|_{m+n_{0}+2} \leq w_{0}\right)$.

Now, let $\mathbf{R}(t, x, \xi ; u)$ be the symbol of $\widetilde{\mathbf{R}}_{\phi}$ in $\underline{(4.21)}$. Then, examining the proof of Lemma 2.4, p. 69 and Theorem 2.2, p. $\underline{293}$ and taking account of (4.64), we have the following lemma for the estimate of $\mathbf{R}$.

Lemma 5.5. Let $w_{0}, T$ be the same as those in Lemma 5.2. Then, there exists a constant $s_{0} \in z_{+}$such that, for any $\alpha, \beta \in \mathbf{Z}_{+}^{n}, \xi \in \mathbf{R}^{n}, 0 \leq t \leq T$, $u \in B^{\infty}\left(\mathbf{R}^{n}\right) \quad\left(\|u\|_{s_{0}} \leq w_{0}\right)$,

$$
\left\|\partial_{x}^{\alpha} \partial_{\xi}^{\beta} \mathbf{R}(t, x, \xi ; u)\right\|_{n_{0}}\langle\xi\rangle^{|\beta|+n-1} \leq c \sum_{k=0}^{|\alpha|}\left(1+\|u\|_{|\alpha|-k+n_{0}+s_{0}}\right)\langle\xi\rangle^{k}
$$

depending only on $w_{0}, T, \alpha, \beta, n_{0}$.

To prove the estimate (3.11) in Lemma 3.3, note that $1-\mathbf{K}(u)$ has an inverse by Treves' inequality (cf. [T, Theorem 0.41]) and the appropriate choice of $\phi_{2}$, $\phi_{3}$ in the definition of $\mathbf{K}$. Consider

$$
\partial_{x}^{\alpha}(1-\mathbf{K}(u)) v=\partial_{x}^{\alpha} g
$$

and rewrite it as

$$
(1-\mathbf{K}(u)) \partial_{x}^{\alpha} v=\left[\partial_{x}^{\alpha}, \mathbf{K}(u)\right] v+\partial_{x}^{\alpha} g .
$$

Then, from what we have seen up to Lemma 5.5 , the estimate can be easily proved by induction on $|\alpha|$ if we apply the following lemma to (5.17).

Lemma 5.6. Let $w_{0}, T$ be the same as those in Lemma 5.2. Then, there exist constants $s_{0} \in \mathbf{Z}_{+}, \delta_{0}>0$ such that, for any $\alpha, \beta \in \mathbf{Z}_{+}^{n}, \xi \in \mathbf{R}^{n}, 0 \leq t \leq T$, $u \in B^{\infty}\left(\mathbf{R}^{n}\right) \quad\left(\|u\|_{s_{0}} \leq w_{0},\left\|u-u_{0}\right\|_{m+n_{0}+1} \leq \delta_{0}\right)$,

$$
\left\|\partial_{\xi}^{\beta} \sigma\left(\left[\partial_{x}^{\alpha}, \mathbf{K}(u)\right]\right)(t, x, \xi)\right\|_{n_{0}}\langle\xi\rangle^{|\beta|+n+1} \leq C \sum_{k=0}^{|\alpha|-1}\left(1+\|u\|_{|\alpha|-k+n_{0}+s_{0}}\right)\langle\xi\rangle^{k}
$$

and

$$
\left\|\left[\partial_{x}^{\alpha}, \mathbf{K}(u)\right] v\right\| \leq C\left(\|u\|_{|\alpha|+s_{0}}\|v\|_{s_{0}}+\|v\|_{|\alpha|-1}\right)
$$

with some constant $C$ depending only on $w_{0}, T, \alpha, \beta, n_{0}$.

(5.18) can be prove by examining the proofs of Theorem 2.1, p. 289 and Theorem 2.2, p. 293 and (5.19) can be proved by the proofs of Lemma 1.4, p. $\underline{57}$, Theorem 1.7, p. 286 and Theorem 2.3, p. 299 with the aid of (5.1) and (5.8). 
Finally, we note about the proof of the estimate (3.9) in Lemma 3.3. Set

$$
\left(D_{x}^{\alpha} \widetilde{\mathbf{E}}_{\phi}(t ; u) G\right)(x)=\int e^{i \phi(t, x, \xi ; u)} \sum_{k=0}^{|\alpha|} \mathbf{d}_{k}(t, x, \xi ; u)\langle\xi\rangle^{k} \hat{G}(\xi) d \xi .
$$

Then, by using (5.9), we can easily proved the following:

Lemma 5.7. Let $w_{0}, T$ be the same as those in Lemma 5.2. Then, there exists a constant $s_{0} \in \mathbf{Z}_{+}$such that, for any $\beta, \gamma \in Z_{+}, \xi \in \mathbf{R}^{n}, 0 \leq t \leq T$, $u \in B^{\infty}\left(\mathbf{R}^{n}\right) \quad\left(\|u\|_{s_{0}} \leq w_{0}\right)$

$$
\left\|\partial_{x}^{\beta} \partial_{\xi}^{\gamma} \mathbf{d}_{k}(t, x, \xi ; u)\right\|_{n_{0}}\langle\xi\rangle^{|\gamma|} \leq C\left(1+\|u\|_{|\alpha|+|\beta|-k+s_{0}}\right)
$$

with some constant $C$ depending only on $w_{0}, T, \alpha, \beta, \gamma$.

Now the estimate (3.9) is a direct consequence of Theorem 2.3, p. 299.

\section{APPENDIX A}

In this appendix, we give some terminologies and formulas used in $\S 4$.

(A.I) Construction of a canonical transformation. Let $U$ be a relatively compact open set of $\mathbf{R}^{n}$ which contains the origin 0 . Let $T^{*} U$ be the cotangent bundle over $U$ and put ${ }^{o} T^{*} U=T^{*} U-\{0\}$. By the triviality of $T^{*} U$, we may write as $T^{*} U=U \times \mathbf{R}^{n}$ and denote by pr: $T^{*} U=U \times \mathbf{R}^{n} \rightarrow U$ the projection map onto the first factor. We introduce fixed coordinates $\left(y^{1}, \ldots, y^{n}, \eta^{1}, \ldots, \eta^{n}\right)$ on $T^{*} U$. Let $\theta$ be the canonical 1-form on $T^{*} U$ which can be expressed as $\theta=\sum_{i=1}^{n} \eta^{i} d y^{i}$ in these coordinates. Given $p_{k}(x, \xi) \in C^{\infty}\left({ }^{o} T^{*} U\right)$, we say that $p_{k}(x, \xi)$ is an of positively homogeneous symbol of degree $k$ if it satisfies $p_{k}(x, \lambda \xi)=\lambda^{k} p_{k}(x, \xi)$ for any $\lambda>0$ and any $(x, \xi) \in{ }^{o} T^{*} U$. We denote by $S_{0}^{k}\left({ }^{0} T^{*} U\right)$ the set of positively homogeneous symbol of degree $k$. Then, we can put the topology on $S_{0}^{k}\left({ }^{o} T^{*} U\right)$ defined by the Fréchet norm; for a positive integer $m>0$,

$$
\|p\|_{(k, 0), m}=\sup _{(y, \eta) \in^{o} T^{*} U,|\alpha|+|\beta| \leq m}|\eta|^{-m+|\beta|}\left|\partial_{y}^{\alpha} \partial_{\eta}^{\beta} p(y, \eta)\right| .
$$

Given $p_{1}(y, \eta) \in S_{0}^{1}\left({ }^{o} T^{*} U\right)$, we recall that $p_{1}(y, \eta)$ is of real principal type on $U$ if it satisfies

( $\left.{ }^{*} 1\right) d p$ and $\theta$ are linearly independent at any $z_{0} \in{ }^{o} T^{*} U$ satisfying $p\left(z_{0}\right)=0$.

Since $\left({ }^{*} 1\right)$ is an open condition on the class $S_{0}^{1}\left({ }^{o} T^{*} U\right)$ in the topology defined by (A.1), we easily get the following:

Lemma A.0. Let $p_{1}(y, \eta) \in S_{0}^{1}\left({ }^{o} T^{*} U\right)$ be of real principal type on $U$. Then there exists a positive constant $\gamma_{0}$ and $k_{0} \in \mathbf{Z}_{+}$, such that for any $\tilde{p}_{1} \in S_{0}^{1}\left({ }^{o} T^{*} U\right)$ with $\left\|\tilde{p}_{1}-p_{1}\right\|_{(1,0), k_{0}}<\gamma_{0}$,

(i) $\tilde{p}_{1}$ is also of real principal type on $U$. 
(ii) If we choose a point $z_{0}\left(; p_{1}\right)=\left(0, \eta_{0}\left(; p_{1}\right)\right) \in{ }^{o} T^{*} U$ such that $p_{1}\left(z_{0}\left(; p_{1}\right)\right)$ $=0$, then there exists a point $z_{0}\left(; \tilde{p}_{1}\right)=\left(0, \eta_{0}\left(; \tilde{p}_{1}\right)\right) \in{ }^{o} T^{*} U$ satisfying $\tilde{p}_{1}\left(z_{0}\left(; \tilde{p}_{1}\right)\right)=0$ and we have

$$
\left|z_{0}\left(; \tilde{p}_{1}\right)-z_{0}\left(; p_{1}\right)\right| \leq C_{0}\left\|\tilde{p}_{1}-p_{1}\right\|_{(1,0), k_{0}}
$$

for some constant $C_{0}>0$.

Remark. From here on the dependence of functions and mappings, etc. on other variables will be emphasized by putting the variables after a semicolon as in $z_{0}\left(; p_{1}\right)$.

Let $\Gamma$ be an open conic set of ${ }^{o} T^{*} U$, i.e., for any $\lambda>0,(x, \lambda \xi) \in \Gamma$ if $(x, \xi) \in \Gamma$. Moreover, we always assume that $\operatorname{pr}(\Gamma)$ is relatively compact. In particular, given a point $w_{0}=\left(x_{0}, \xi_{0}\right) \in{ }^{o} T^{*} U$, and positive constants $r_{1 x}, r_{1 \xi}$, we put $\Gamma_{w_{0}}\left(r_{1 x}, r_{1 \xi}\right)=\left\{(x, \xi)|| x-x_{0}\left|<r_{1 x},\right| \xi /|\xi|-\xi_{0} /\left|\xi_{0}\right| \mid<r_{1 \xi}\right\}$. The definition of positive homogeneity still makes sense for symbols defined on an open conic subset $\Gamma$. We denote by $S_{0}^{k}(\Gamma)$ the set of positively homogeneous symbols of degree $k$ which can be extended smoothly to the closure of $\operatorname{pr}(\Gamma)$ and by $\|\cdot\|_{(k, 0), m, \Gamma}$ the seminorms on $S_{0}^{k}(\Gamma)$ given by restricting the seminorms (A.1) to $\Gamma$.

Let $\chi$ be a local diffeomorphism from $\Gamma$ into $T^{*} U$ such that $\chi$ can be extended smoothly to the closure of $\operatorname{pr}(\Gamma)$. In local coordinates, we may write $\chi$ as

$$
\chi(x, \xi)=(y(x, \xi), \eta(x, \xi))
$$

for $(x, \xi) \in \Gamma . \chi$ is called a canonical transformation of $\Gamma$ of positively homogeneous degree one if it satisfies for any $\lambda>0$, and any $(x, \xi) \in \Gamma$,

$$
\chi^{*} \Omega=\Omega \text { and }(y(x, \lambda \xi), \eta(x, \lambda \xi))=(y(x, \xi), \lambda \eta(x, \xi))
$$

where $\Omega$ is the canonical 2-form defined by $\Omega=\sum d \xi^{i} \Lambda d x^{i}$.

For a canonical transformation $\chi$ of positively homogeneous degree one as above, we define a norm of $\chi$, for any $s \in \mathbf{Z}_{+}$, by

$$
|\chi|_{s, \Gamma}=\sup _{|\alpha|+|\beta| \leq s,(x, \xi) \in \Gamma}\left\{|\xi|^{|\beta|}\left|\partial_{x}^{\alpha} \partial_{\xi}^{\beta} y(x, \xi)\right|+|\xi|^{|\beta|-1}\left|\partial_{x}^{\alpha} \partial_{\xi}^{\beta} \eta(x, \xi)\right|\right\}
$$

Now we prove the following:

Theorem A.1. Let $p_{1}(y, \eta) \in S_{0}^{1}\left({ }^{o} T^{*} U\right)$ be of real principal type on $U$. Fix a point $z_{0}=\left(0, \eta_{0}\right) \in{ }^{o} T^{*} U$ satisfying $p_{1}\left(z_{0}\right)=0$ and a point $\left(0, e_{n}\right) \in$ ${ }^{o} T^{*} U, e_{n}=(0, \ldots, 1)$.

(i) There exist positive constants $\bar{r}_{1 x}, \bar{r}_{1 \xi}, \bar{r}_{1 y}, \bar{r}_{1 \eta}$, and a canonical transformation $\chi(; p): \Gamma_{\left(0, e_{n}\right)}\left(\bar{r}_{1 x}, \bar{r}_{1, \xi}\right) \rightarrow \Gamma_{z_{0}}\left(\bar{r}_{1 y}, \bar{r}_{1 \eta}\right)$ of positively homogeneous degree one such that

(ia) $\chi\left(; p_{1}\right)\left(\left(0, e_{n}\right)\right)=z_{0}$;

(ib) $\left(\chi\left(; p_{1}\right)^{*} p_{1}\right)(x, \xi)=\xi^{1}$. 
(ii) There exist $k_{1} \in \mathbf{Z}_{+}, \gamma_{1}, 0$ such that for any given $s_{1} \in \mathbf{Z}_{+}$, the following properties hold: For any $\tilde{p}_{1} \in S_{0}^{1}\left({ }^{o} T^{*} U\right)$ satisfying $\left\|\tilde{p}_{1}-p_{1}\right\|_{(1,0), k_{1}}<\gamma_{1}$, there exist a point $z_{0}\left(; \tilde{p}_{1}\right)=\left(0, \eta_{0}\left(; \tilde{p}_{1}\right)\right) \in{ }^{o} T^{*} U$ and a canonical transformation $\chi\left(; \tilde{p}_{1}\right): \Gamma_{\left(0, e_{n}\right)}\left(\bar{r}_{1 x}, \bar{r}_{1 \xi}\right) \rightarrow \Gamma_{z_{0}}\left(\bar{r}_{1 y}, \bar{r}_{1 \eta}\right)$ of positively homogeneous degree one satisfying:

(iia) $z_{0}\left(; \tilde{p}_{1}\right)$ satisfies $\chi\left(; \tilde{p}_{1}\right)\left(\left(0, e_{n}\right)\right)=z_{0}\left(; \tilde{p}_{1}\right)$ and $\left|z_{0}\left(; \tilde{p}_{1}\right)-z_{0}\left(; p_{1}\right)\right| \leq$ $C_{0}\left\|\tilde{p}_{1}-p_{1}\right\|_{(1,0), k_{1}}$

(iib) $\left(\chi\left(; \tilde{p}_{1}\right)^{*} \tilde{p}_{1}\right)(x, \xi)=\xi^{1}$;

(iic) $\chi\left(; \tilde{p}_{1}\right)$ satisfies

$$
\left|\chi\left(; \tilde{p}_{1}\right)-\chi\left(; p_{1}\right)\right|_{s_{1}, \Gamma_{\left(0, n_{n}\right)}\left(p_{1, x}, p_{1 \xi}\right)} \leq C_{s_{1}}\left\|\tilde{p}_{1}-p_{1}\right\|_{(1,0), s_{1}+k_{1}}
$$

for some $C_{s_{1}}>0$.

To prove Theorem A.1, we begin with some basic lemmas. Let $p_{1}(y, \eta) \in$ $S_{0}^{1}\left({ }^{o} T^{*} U\right)$. Then in terms of the local coordinates $(y, \eta)$, the Hamiltonian vector field $H_{p_{1}}$ is given by

$$
H_{p_{1}}=\sum_{i=1}^{n} \frac{\partial p_{1}}{\partial \eta^{i}} \frac{\partial}{\partial y^{i}}-\sum_{i=1}^{n} \frac{\partial p_{1}}{\partial y^{i}} \frac{\partial}{\partial \eta^{i}}
$$

on ${ }^{o} T^{*} U$. Since $H_{p_{1}}$ is of real principal type at $z_{0}, H_{p_{1}}$ is not parallel to the radial vector field $\Xi=\sum_{i=1}^{n} \eta^{i} \partial / \partial \eta^{i}$.

Let $S^{*} U$ be the unit sphere bundle defined by the equivalence relation

$$
(y, \eta) \sim\left(y^{\prime}, \eta^{\prime}\right) \leftrightarrow y=y^{\prime}, \eta=\lambda \eta^{\prime} \text { for some } \lambda>0 .
$$

and denote by $\hat{\pi}$ the projection $\hat{\pi}:{ }^{\circ} T^{*} \mathbf{R}^{n} \rightarrow S^{*} \mathbf{R}^{n}$.

Fix a point $z_{0} \in{ }^{\circ} T^{*} U, z_{0}=\left(0, \eta_{0}\right)$ and put $\hat{\pi}\left(z_{0}\right)=\hat{z}_{0}$. Take an open conic neighborhood $\Gamma$ of $z_{0}$ and a neighborhood $\hat{\Gamma}$ of $\hat{z}_{0}$ such that $\hat{\pi}(\Gamma)=$ $\hat{\Gamma}$. By changing the index of the $\eta$-variables we may assume that $\eta^{n} \neq 0$ on $\Gamma$ and introduce coordinates $\left(y^{1}, \ldots, y^{n}, \hat{\eta}^{1}, \ldots, \hat{\eta}^{n-1}\right)$ around $\hat{z}_{0}$. Then $\Gamma=\hat{\Gamma} \times \mathbf{R}_{+}$, and the projection $\hat{\pi}:{ }^{o} T^{*} \mathbf{R}^{n} \rightarrow S^{*} \mathbf{R}^{n}$ can be expressed by

$$
\hat{\eta}^{a}=\eta^{a} / \eta^{n}(a=1, \ldots, n-1) .
$$

Put $\eta^{n}=t$ and consider the contact 1 -form $\omega$ on $S^{*} U$ defined by

$$
\omega=d y^{1}+\sum_{a=2}^{n} \hat{\eta}^{a} d y^{a}
$$

in terms of the local coordinates $(y, \hat{\eta})$. Then we have $\theta=t \omega$ and $\omega$ satisfies $(d \omega)^{n} \Lambda \omega \neq 0$.

By the homogeneity of $p_{1}, H_{p_{1}}$ can be projected down by $\hat{\pi}$ to define a contact vector field $\hat{u}$ on $S^{*} \mathbf{R}^{n}$. Here the vector field $\hat{u}$ on $S^{*} \mathbf{R}^{n}$, the contact vector field, satisfies

$$
\left.\left.L_{\hat{u}} \omega=d(\omega\lrcorner \hat{u}\right)+d \omega\right\lrcorner \hat{u}=f \omega
$$


for some smooth function $f$, where $L_{\hat{u}}$ is the Lie derivative with respect to $\hat{u}$. We also define a smooth vector field $\hat{\xi}_{\omega}$ on $S^{*} \mathbf{R}^{n}$ by

$$
\left.\omega\lrcorner \hat{\xi}_{\omega} \equiv 1, \quad d \omega\right\lrcorner \hat{\xi}_{\omega} \equiv 0 .
$$

$\hat{\xi}_{\omega}$ is called the characteristic vector field for the contact structure $\omega$. Moreover, we define the subbundle $\mathbf{E}_{\omega}$ by the solution of $\omega=0$. Let $\widehat{\mathbf{E}}_{\omega}$ be the annihilator of $\hat{\xi}_{\omega}$ in the cotangent bundle of $S^{*} \mathbf{R}^{n}$. Then, we have

$$
T\left(S^{*} \mathbf{R}^{n}\right)=\mathbf{R} \hat{\xi}_{\omega} \oplus \mathbf{E}_{\omega}, \quad T^{*}\left(S^{*} \mathbf{R}^{n}\right)=\mathbf{R} \omega \oplus \widehat{\mathbf{E}}_{\omega} .
$$

The mapping $d \omega: \hat{v} \rightarrow d \omega\lrcorner \hat{v}$ is an isomorphism of $\mathbf{E}_{\omega}$ onto $\widehat{\mathbf{E}}_{\omega}$. We denote by $d \omega^{-1}$ its inverse mapping.

Given a pair $(f, \hat{u})$ consisting of a smooth function $f$ and a contact vector field $\hat{u}$ on $S^{*} \mathbf{R}^{n}$, satisfying $L_{\hat{u}} \omega=f \omega$, we write

$$
\hat{u}=q \hat{\xi}_{\omega}+\hat{v}, \quad \hat{v} \in \Gamma\left(\mathbf{E}_{\omega}\right),
$$

where $\Gamma\left(\mathbf{E}_{\omega}\right)$ denotes the smooth sections of $\mathbf{E}_{\omega}$. Then we have

$$
\left.L_{\hat{u}} \omega+f \omega=d q+d \omega\right\lrcorner v+f \omega=0 .
$$

Thus $\hat{\xi}_{\omega} q=f$, and we see that $d q-\left(\hat{\xi}_{\omega} q\right) \omega$ is contained in $\Gamma(\widehat{\mathbf{E}} \omega)$ for any smooth function $q$. Therefore, we have the following correspondence:

$$
q \leftrightarrow\left(-\hat{\xi}_{\omega} q, \hat{u}_{q}=\left(\hat{\xi}_{\omega} q\right) \hat{\xi}_{\omega}+d \omega^{-1}\left(d q-\left(\hat{\xi}_{\omega} q\right) \omega\right)\right) .
$$

Putting

$$
\begin{aligned}
q(y, \hat{\eta}) & =p_{1}\left(y^{1}, \ldots, y^{n}, \hat{\eta}^{1}, \ldots, \hat{\eta}^{n-1}, 1\right), \\
p_{1}(y, \eta) & =t q\left(y^{1}, \ldots, y^{n}, \eta^{1} / \eta^{n}, \ldots, \eta^{n-1} / \eta^{n}\right)
\end{aligned}
$$

for $p_{1}(y, \eta) \in S_{0}^{1}(\Gamma)$ and $q(y, \hat{\eta}) \in C^{\infty}(\widehat{\Gamma})$ by using the coordinates in $\Gamma$ and $\widehat{\Gamma}$, we get a correspondence between the Hamiltonian vector field on $\Gamma$ and the contact vector field on $\hat{\Gamma}$. Using the local coordinates $(y, \hat{\eta})$, it is easily seen that $\hat{u}_{q}$ and $H_{p_{1}}$ can be written in the form:

$$
\hat{u}_{q}=-\sum_{a=1}^{n-1} \frac{\partial q}{\partial \hat{\eta}^{a}} \frac{\partial}{\partial y^{a}}-\sum_{a=1}^{n-1}\left\{\frac{\partial q}{\partial y^{a}}-\hat{\eta}^{a} \frac{\partial q}{\partial y^{n}}\right\} \frac{\partial}{\partial \hat{\eta}^{a}}+\left\{\sum_{a=1}^{n-1} q-\frac{\hat{\eta}^{a}}{2} \frac{\partial q}{\partial \hat{\eta}^{a}}\right\} \frac{\partial}{\partial y^{n}}
$$

and

$$
H_{p_{1}}=\hat{u}_{q}+t \frac{\partial q}{\partial y^{n}} \frac{\partial}{\partial t} .
$$

By this correspondence we easily get the following lemma. 
Lemma A.2. Let $p_{1} \in S_{0}^{1}(\Gamma)$ and assume that $p_{1}(x, \xi)$ is of real principal type at $z_{0}$. Then $\hat{u}_{q}$ does not vanish at $\hat{z}_{0}$ and $\left.\left(d y^{n}\right\lrcorner u_{q}\right)\left(\hat{z}_{0}\right)=0$.

Now we put a topology in $C^{\infty}\left(S^{*} U\right)$ by the Fréchet norm $\|q\|_{k}$ : for positive integers $k$,

$$
\|q\|_{k}=\sup _{|\alpha|+|\beta| \leq k,(y, \hat{\eta}) \in S^{*} U}\left|\partial_{y}^{\alpha} \partial \hat{\eta}^{\beta} q(y, \eta)\right| .
$$

The restriction of the seminorm (A.15) to the open subset $\widehat{\Gamma}$ of $S^{*} U$ is denoted by $\|\cdot\|_{k, \widehat{\Gamma}}$.

Denote by $V$ a $2 n-2$ dimensional subspace which is transversal to $\hat{u}_{q}\left(\hat{z}_{0}\right)$. Notice that for any $\tilde{p}_{1}(x, \xi) \in S_{0}^{1}\left({ }^{o} T^{*} \mathbf{R}^{n}\right)$ which is sufficiently close to $p_{1}$ in the norm (A.1), $V$ is also transversal to $\hat{u}_{\tilde{q}_{1}}\left(\hat{z}_{0}\right)$.

For positive $r_{1 y}, r_{1 \hat{\eta}}$, we shall denote by $\widehat{\Gamma}_{\hat{z}_{0}}\left(r_{1 y}, r_{1 \hat{\eta}}\right)$ the open neighborhood of $\hat{z}_{0}$ defined by

$$
\widehat{\Gamma}_{\hat{z}_{0}}\left(r_{1 y}, r_{1 \hat{\eta}}\right)=\left\{\hat{z}=(y, \hat{\eta})|| y-y_{0}\left|<r_{1 y},\right| \hat{\eta}-\hat{\eta}_{0} \mid<r_{1 \hat{\eta}}\right\} .
$$

Proposition A.3. In the situation of Lemma A.2

(i) there exist positive constants $\bar{r}_{3 y}, \bar{r}_{3 \hat{\eta}}, \bar{r}_{3 Y}, \bar{r}_{3 \widehat{E}}, a C^{\infty}$ diffeomorphism $\Phi(; q)=\Phi(Y, \widehat{E})=(y(Y, \widehat{E}), \hat{\eta}(Y, \widehat{E})): \widehat{\Gamma}_{(0,0)}\left(\bar{r}_{3 Y}, \bar{r}_{3 \widehat{E}}\right) \rightarrow \widehat{\Gamma}_{\hat{z}_{0}}\left(\bar{r}_{3 y}, \bar{r}_{3 \hat{\eta}}\right)$, and $a$ smooth function $\bar{F}$ on $\left.\widehat{\gamma}_{(0,0}\right)\left(\bar{r}_{3 Y}, \bar{r}_{3 \widehat{E}}\right)$ such that

(ia) $\Phi(; q)\left(\hat{z}_{0}\right)=(0, \ldots, 0,0, \ldots, 0)$;

(ib) $\Phi(; q)^{*}\left(\frac{\partial}{\partial Y^{1}}\right)=\hat{u}_{q}$;

(ic) $\Phi(; q)^{*} \omega=\bar{F}\left(d Y^{n}+\sum_{a=2}^{n} \widehat{E}^{a} d Y^{a}\right)$.

(ii) There exist $k_{3} \in \mathbf{Z}_{+}, \gamma_{3}>0$ such that for any given $s_{3} \in \mathbf{Z}_{+}$the following properties hold: for any $\tilde{q}=\tilde{p}_{1}\left(y, \hat{\eta}^{1}, \ldots, \hat{\eta}^{n-1}, 1\right)$ satisfying $\|\tilde{q}-q\|_{k_{3} \hat{\Gamma}}<$ $\gamma_{3}$, there exist a point $\tilde{z}_{0}(; \tilde{q})=\left(0, \tilde{\eta}_{0}(; \tilde{q})\right) \in S^{*} U$, a $C^{\infty}$ diffeomorphism $\Phi(Y, \widehat{E} ; \tilde{q})=(y(Y, \widehat{E} ; \tilde{q}), \eta(Y, \widehat{E} ; \tilde{q})): \widehat{\Gamma}_{(0,0)}\left(\bar{r}_{3 Y}, \bar{r}_{3 \widehat{E}}\right) \rightarrow \widehat{\Gamma}_{\hat{z}_{0}}\left(\bar{r}_{3 y}, \bar{r}_{3 \hat{\eta}}\right)$ and $a$ smooth function $\bar{F}(; \tilde{q})$ on $\Gamma_{(0,0)}\left(\bar{r}_{3 Y}, \bar{r}_{3 \widehat{E}}\right)$ such that

(iia) (ia-c) still hold with $\hat{z}_{0}, \Phi, \bar{F}$ replaced by $\tilde{z}_{0}(; \tilde{q}), \Phi(; \tilde{q})$ and $\bar{F}(; \tilde{q})$ respectively.

(iib) For some constant $C_{3}^{\prime}$, we have

$$
\left|\hat{\tilde{z}}_{0}(; \tilde{q})-\hat{z}_{0}\right| \leq C_{3}^{\prime}\|\tilde{q}-q\|_{k_{3}, \widehat{\Gamma}_{\Sigma_{0}}\left(r_{33}, r_{3 \tilde{i}}\right)} .
$$

(iid) $\Phi(Y, \widehat{E} ; \tilde{q})=(y(Y, \widehat{E} ; \tilde{q}), \eta(Y, \widehat{E} ; \tilde{q}))$ and $\bar{F}(; \tilde{q})$ satisfy

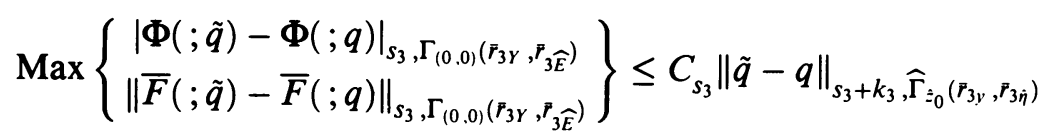

for some constant $C_{s_{3}}>0$.

Proof. Let $V$ be a hyperplane which is transversal to $u_{q}\left(\hat{z}_{0}\right)$. Using Lemma A.2, there exists a linear change of coordinates $\left(y^{\prime}, \hat{\eta}^{\prime}\right)$ of $(h, \eta)$ 
satisfying the following properties: (0) $z_{0}=(0, \ldots, 0)$, (i) $y^{\prime n}=y^{n}$, (ii) $d y^{n}+\sum_{a=1}^{n-1} \hat{\eta}^{a} d y^{a}=d y^{\prime n}+\sum_{a=1}^{n-1} \hat{\eta}^{\prime a} d y^{a}$, (iii) $V=\left\{\left(0, y^{\prime 2}, \ldots, \hat{\eta}^{\prime n-1}\right)\right\}$, and (iv) $u_{q}$ can be expressed as $\partial / \partial y^{\prime 1}$ at $\hat{z}_{0}$. Since this coordinate change can be controlled under perturbation of $q$ to $\tilde{q}$, the coordinate $\left(y^{\prime}, \hat{\eta}^{\prime}\right)$ satisfies the properties in Proposition A.3. Thus we may assume that the coordinates $(y, \eta)$ satisfy properties (i)-(iv). For a point $z^{\prime}=\left(0, Y^{2}, \ldots, Y^{n}, \widehat{E}^{2}, \ldots, \widehat{E}^{n}\right)$, we define the mapping

$$
\Phi\left(Y^{1}, Y^{2}, \ldots, Y^{n}, \widehat{E}^{2}, \ldots, \hat{E}^{n}\right)=\left(\exp Y^{1} u_{q}\right)\left(z^{\prime}\right)=\left(y^{1}, \ldots, y^{n}, \hat{\eta}^{2}, \ldots, \hat{\eta}^{n}\right)
$$

where $Z(t)=\left(\exp t u_{q}\right)\left(z^{\prime}\right)$ is the integral curve of $u_{q}$ defined by the solution of the ordinary differential equation:

$$
\frac{\partial Z(t)}{\partial t}=u_{q}(Z(t)), \quad Z(0)=z^{\prime} .
$$

Then we have

$$
\Phi\left(\hat{z}_{0}\right)=0, \quad d \Phi\left(\hat{z}_{0}\right)=\mathrm{id} .
$$

Therefore, by the inverse function theorem, there exists positive constants $\bar{r}_{3 y}$, $\bar{r}_{3 \hat{\eta}}, \bar{r}_{3 Y}$, and $\bar{r}_{3 \widehat{E}}$ such that $\Phi$ is a $C^{\infty}$ diffeomorphism from $\widehat{\Gamma}_{(0,0)}\left(\vec{r}_{3 Y}, \bar{r}_{3 \widehat{E}}\right)$ onto $\widehat{\Gamma}_{\hat{z}_{0}}\left(\bar{z}_{3 y}, \bar{r}_{3 \hat{\eta}}\right)$. Thus we get (i). If we take $\tilde{q}$ sufficiently close to $q$, then $V$ is also transversal to $u_{\tilde{q}}$ at the origin. Thus, by using the smooth dependency of solution on parameters for the ordinary differential equation (A.17), we get (ii) in Proposition A.3.

Now we study $\Phi^{*} \hat{u}_{q}$ and $\Phi^{*} \omega$ in the coordinates $\left\{Y^{1}, \ldots, \hat{E}^{n}\right\}$ defined on the set $\widehat{\Gamma}_{(0,0)}\left(\bar{r}_{3 Y}, \bar{r}_{3 \widehat{E}}\right)$. In these coordinates, we can rewrite the contact 1-form $\omega$ as

$$
\Phi^{*} \omega=\omega_{n}(; q) d Y^{n}(; q)+\sum_{i=1}^{n-1} \omega_{i}(; q) d Y^{i}(; q)+\sum_{a=2}^{n} \omega_{a}(; q) d \widehat{E}^{a}(; q)
$$

From the proof of Proposition A.3(ii), $\omega_{n}(0,0) \neq 0$ and we may assume that $\omega_{n} \neq 0$ on $\widehat{\Gamma}_{(0,0)}\left(\bar{r}_{3 Y}, \bar{r}_{3 \widehat{E}}\right)$.

Since $L_{u_{q}} \omega=h(; q) \omega$ and $u_{q}=\partial / \partial Y^{1}$, we have

$$
\begin{aligned}
\omega_{i}(; q)= & \omega_{i}(; q)\left(0, Y^{2}(; q), \ldots, Y^{n}(; q), \widehat{E}^{2}(; q), \ldots, \widehat{E}^{n}(; q)\right) \\
& \cdot \exp \int_{0}^{Y^{1}(; q)} h(; q)\left(t, Y^{2}(; q), \ldots, \hat{E}^{n}(; q)\right) d t, \\
\omega_{a}(; \tilde{q})= & \omega_{a}(; \tilde{q})\left(0, Y^{2}(; q), \ldots, Y^{n}(; q), \widehat{E}^{2}(; q), \ldots, \widehat{E}(; q)\right) \\
& \cdot \exp \int_{0}^{Y^{1}(; q)} h(; q)\left(t, Y^{2}(; q), \ldots, \hat{E}^{n}(; q)\right) d t .
\end{aligned}
$$

Put

$$
\bar{F}(; q)=\exp \int_{0}^{Y^{\prime}(; q)} h(; q)\left(t, Y^{2}(; q), \ldots, \hat{E}^{n}(; q)\right) d t, \quad \omega(; q)=\bar{F}(; q) \omega^{\prime}\left(^{\prime} q\right),
$$


where $\omega^{\prime}=d Y^{n}+\sum_{i=1}^{n-1} \omega_{i}^{\prime} d Y^{i}+\sum_{a=1}^{n} \omega_{a}^{\prime} d \widehat{E}^{a}$ and the coefficients of $\omega^{\prime}$ do not contain the variable $Y^{1}(; \tilde{q})$.

Now we shall rewrite $\omega^{\prime}$ into a canonical contact form. Denote by $\xi_{\omega^{\prime}}$ the characteristic vector field for $\omega^{\prime}$. Then it is clear that the coefficients of $\xi_{\omega^{\prime}}$ do not involve the variable $Y^{1}$ and $\left[\xi_{\omega^{\prime}}, u_{q}\right]=0$. Hence, as in the proof of Proposition A.3(ii), there exists local coordinates $\left(Y^{\prime \prime}, \ldots, Y^{\prime n}, \widehat{E}^{\prime 2}, \ldots, \widehat{E}^{\prime n}\right)$ such that $u_{q}=\partial / \partial Y^{\prime 1}$, and $\xi_{\omega^{\prime}}=\partial / \partial Y^{\prime n}$. Furthermore, we write $\omega^{\prime}(; \tilde{q})$ in terms of the newly chosen coordinates $\left\{Y^{\prime 1}(; q), \ldots, Y^{\prime n}(; q), \widehat{E}^{\prime 2}(; q), \ldots, \widehat{E}^{\prime n}(; q)\right\}$ as

$$
\omega^{\prime}(; q)=d Y^{\prime n}(; q)+\sum_{i=1}^{n-1} \omega_{i}^{\prime \prime}(; q) d Y^{i}(; q)+\sum_{a=2}^{n} \omega_{a}^{\prime \prime}(; q) d \widehat{E}^{i}(; q)
$$

and $\omega_{1}^{\prime \prime}(0,0)=0$. Again $\omega_{i}^{\prime \prime}, \omega_{a}^{\prime \prime}$ do not contain the variable $Y^{1}$, since $L_{\boldsymbol{\xi}_{\omega^{\prime}}} \omega^{\prime}=0$.

Now $d \omega^{\prime}$ can be regarded as a symplectic 2-form on a neighborhood of the origin of $\mathbf{R}^{2 n-2}$ and $L_{u_{q}} d \omega^{\prime}=0$. Then $d \omega^{\prime}$ can be written by $d \omega^{\prime}=\Delta_{1}+\Delta_{2}$, where

$$
\Delta_{1}=d Y^{\prime 1} \wedge\left(\sum_{j=2}^{n-1} \Lambda_{1 j} d Y^{\prime j}+\sum a=2^{n} \Delta_{1 a} d \widehat{E}^{\prime a}\right)
$$

and

$$
\begin{aligned}
\Delta_{2}= & \sum_{2 \leq i, j \leq n-1} \Delta_{2 i j} d Y^{\prime i} \wedge d Y^{\prime j} \\
& +\sum_{2 \leq i \leq n-1,2 \leq a \leq n} \Delta_{2 i a} d Y^{\prime i} \wedge d \widehat{E}^{\prime a}+\sum_{2 \leq a, b \leq n} \Delta_{2 a b} d \widehat{E}^{\prime a} \wedge d \widehat{E}^{\prime b} .
\end{aligned}
$$

Since $d^{2} \omega^{\prime}=0$, and $d \omega^{\prime}$ has rank $2 n-2$, we see that $d \Delta_{1}=0$ and $\Delta_{2}$ is a closed form of rank $2 n-4$ on a neighborhood of the origin of $\mathbf{R}^{2 n-2}$. Therefore there is $C^{\infty}$ function $\lambda_{2}$ such that $\Delta_{1}=d Y^{\prime 1} \wedge d \lambda_{2}$.

Now we apply Darboux's Lemma. Namely, we consider the standard closed 2-form $\Delta_{*}$ on $D^{\prime \prime}\left(\vec{r}_{4 x}^{\prime}, \vec{r}_{4 \hat{\xi}}^{\prime}\right)=\left\{\left(Y^{2}, \ldots, Y^{n-1}, \widehat{E}^{\prime 2}, \ldots, \widehat{E}^{\prime n-1}\right)|| Y\left|<r_{4 x}^{\prime},\right| \widehat{E}^{\prime} \mid<\right.$ $\left.r_{4 \hat{\xi}}^{\prime}\right\}$ :

$$
\Delta_{*}=\sum_{a=2}^{n-1} d Y^{a} \wedge E^{a}
$$

By a linear symplectic transformation of $(Y, E)$, we may assume that $\Delta_{2}(; q)(0,0)=\Delta_{*} ;$ this linear symplectic transformation depends smoothly on $\tilde{q}$ in the matrix topology.

Let $\Delta_{t}(; \tilde{q})=\Delta_{*}+t\left(\Delta_{2}(; \tilde{q})-\Delta_{*}\right), 0 \leq t \leq 1$. For each $t, \Delta_{t}(; \tilde{q})(0,0)=\Delta_{*}$ is nondegenerate. Hence, there is a neighborhood $D^{\prime \prime}\left(\vec{r}_{4}^{\prime \prime}\right)$ of $(0,0) \in \mathbf{R}^{2 n-4}$ on which $\Delta_{t}(; \tilde{q})$ is nondegenerate for all $0 \leq t \leq 1$. Thus, by the Poincaré 
lemma, $\Delta(; \tilde{q})=d(\alpha(; \tilde{q}))$ for a 1-form $\alpha(; \tilde{q})$. Namely, define $\alpha(; \tilde{q})=$ $\sum_{l=1}^{2 n-4} \alpha_{l}(; \tilde{q})(u) d u^{l}$ by

$$
\alpha^{l}(; \tilde{q})(u)=\int_{0}^{1} t \Delta(; \tilde{q})(t u)\left(u, e_{l}\right) d t
$$

at a point $u=\left(x^{3}, \ldots, \hat{\xi}^{n}\right)$, where $e_{l}$ is the unit vector tangent to the $Y$ or $E$-axis. We may assume $\alpha(; \tilde{q})(0,0)=0$. Define a smooth vector field $Z(t)$ by $\left.\Delta_{t}(; \tilde{q})\right\lrcorner Z(; \tilde{q})(t)=\alpha(; \tilde{q})$, which is possible since $\Delta_{t}(; \tilde{q})$ is nondegenerate. Moreover, since $Z(t)(; \tilde{q})(0,0)=(0,0)$ and by the local existence theory, there exists a positive $\vec{r}_{5}^{\prime}$ and a flow $\mathbf{F}_{t}$ of $Z(; \tilde{q})(t)$ on $D^{\prime \prime}\left(\vec{r}_{5}^{\prime}\right)$. Thus we get

$$
\frac{d}{d t}\left(\mathbf{F}_{t}^{*} \Delta_{t}(; \tilde{q})\right)=\mathbf{F}_{t}^{*}(-d \alpha(; \tilde{q})+\Delta(; \tilde{q}))=0 .
$$

Therefore $\mathrm{F}_{1}^{*} \Delta_{1}(; \tilde{q})=\Delta_{*}$. Thus we have

$$
d \omega^{\prime}=d Y^{\prime 1} \wedge d \lambda+\sum_{b=2}^{n-1} d \widehat{E}^{\prime b} \wedge d Y^{\prime b},
$$

and $\partial \lambda / \partial \widehat{E}^{\prime 1} \neq 0$. This means $\left\{Y^{\prime}, \lambda, \widehat{E}^{\prime 2}, \ldots, \hat{E}^{\prime n-1}\right\}$ is a coordinate around the origin which we denote by $\left\{Y^{\prime}, \widehat{E}^{\prime}\right\}$. Then $\omega^{\prime}=d Y^{n}+f+\sum_{a=2}^{n} \widehat{E}^{\prime a} d Y^{\prime a}$, where $f=f(Y, E)$. Put $Y^{n}=Y^{\prime n}+f$. Then $\left\{Y^{\prime 1}, \ldots, Y^{\prime n}, \widehat{E}^{\prime}\right\}$ satisfies Proposition A.3. Checking the parameter dependence of the ordinary differential equations of type (A.17) and the proof of the Darboux and Poincare Lemmas, we get (ii) in Proposition A.3.

Now Theorem A.1 is obvious. In fact, taking a local diffeomorphism $\phi(y, \hat{\eta})$ $=\left(Y^{\prime}, \widehat{E}^{\prime}\right)$ given by Proposition A.3 and putting $\phi(y, \eta)=\left(Y^{\prime}, \eta^{n} \widehat{E}^{\prime 1}, \ldots\right.$, $\left.\eta^{n} \widehat{E}^{\prime n-1},(1 / \bar{F}) \eta^{n}\right)$, we have $\phi^{*} \theta=\theta$, where $\theta=\sum \xi^{i} d x^{i}$, which gives Theorem A.1.

(A.II) Remarks on pseudodifferential operators. Let $U_{x}$ and $U_{y}$ be relatively compact open sets of $\mathbf{R}^{n}$ which contain the origin 0 , with coordinates $\left(x^{1}, \ldots\right.$, $\left.x^{n}\right)$ and $\left(y^{1}, \ldots, y^{n}\right)$ respectively. Put $\Lambda_{x y}=U_{x} \times{ }^{o} T^{*} U_{y}=U_{x} \times U_{y} \times$ $\left\{\mathbf{R}^{n}-\{0\}\right\}$. Denote by $S^{m}\left(\Lambda_{x y}\right)$ the set of all $C^{\infty}$ functions $p(x, y, \eta)$ on $\tilde{\Lambda}_{x y} \equiv U_{x} \times T^{*} U_{y}$ such that $\operatorname{supp} p \subset \tilde{\Lambda}_{x y}$ and $p$ has the asymptotic expansion

$$
p(x, y, \eta) \sim \sum_{k=0}^{\infty} p_{k}(x, y, \eta)
$$

where $p_{k}(x, y, \eta)$ is a smooth function on $\tilde{\Lambda}_{x y}$ homogeneous of degree $=m-k$ in the $\eta$-variables for $|\eta| \geq 1$. Here $\sim$ means that for any $N \in \mathbf{Z}_{+}, p-\sum_{m}^{1-N} p_{k}$ satisfies the estimate (A.22) given below with $m$ replaced by $m-N$. Usually such a $p(x, y, \eta)$ is called a classical symbol of order $m . p_{m}(x, y, \eta)$ is called the principal symbol of $p(x, y, \eta)$. For any multi-indices $\alpha, \beta, \gamma, p(x, y, \eta) \in$ $S^{m}\left(\Lambda_{x y}\right)$ satisfies

$$
\left|\partial_{x}^{\alpha} \partial_{\xi}^{\beta} \partial_{y}^{\gamma} p(x, y, \eta)\right| \leq C_{\alpha \beta \gamma}(1+|\eta|)^{m-|\beta|} .
$$


These definitions still make sense for functions defined on an open conic subset of $\Lambda_{x y}$. Notice that $S^{m}\left(\Lambda_{x y}\right)$ is a Fréchet space when equipped with the topology defined by the seminorms:

$$
\text { (A.23) }\|p\|_{m, k}=\sup _{|\alpha|+|\beta|+|\gamma| \leq k,(x, y, \eta) \in \Lambda_{x, y}}(1+|\eta|)^{-m+|\beta|}\left|\partial_{x}^{\alpha} \partial_{\xi}^{\beta} \partial_{y}^{\gamma} p(x, y, \eta)\right| .
$$

Put $S^{\infty}\left(\Lambda_{x y}\right)=\bigcup S^{m}\left(\Lambda_{x y}\right)$ equipped with the direct limit topology, and set $S^{-\infty}\left(\Lambda_{x y}\right)=\bigcap S^{m}\left(\Lambda_{x y}\right)$. Denote by $S_{0}^{m}\left(\Lambda_{x y}\right)$ the set of all $C^{\infty}$ functions $p(x, y, \eta)$ on $\Lambda_{x y}$ of positively homogeneous degree $m$ such that $\operatorname{supp} p \subset$ $\tilde{\Lambda}_{x y}$. As in (A.23), we can define the seminorms $\|\cdot\|_{m, k}$ on $S_{0}^{m}\left(\Lambda_{x y}\right)$ by replacing $1+|\eta|$ with $|\eta|$ in (A.23). Given $m$, we can define the direct sum $\bigoplus_{k=m}^{m-N} S_{0}^{k}\left(\Lambda_{x y}\right)$ with the induced topology. Moreover we have the direct limit topology on $\bigoplus_{k \leq m} S_{0}^{k}\left(\Lambda_{x y}\right)$.

Setting $\Lambda_{y}={ }^{o} T^{*} U_{y}$, we denote by $S^{m}\left(\Lambda_{y}\right), S_{0}^{m}\left(\Lambda_{y}\right)$ the subset of $S^{m}\left(\Lambda_{x y}\right)$, $S_{0}^{m}\left(\Lambda_{x y}\right)$ whose elements $p(y, \eta)$ do not contain $x$-variables. $S_{0}^{m}\left(\Lambda_{y}\right)$ coincides with $S_{0}^{m}\left({ }^{o} T^{*} U_{y}\right)$ defined in (A.I). The topology of $S_{*}^{m}\left(\Lambda_{y}\right)$ is given by the induced topology from $S_{*}^{m}\left(\Lambda_{x y}\right)$. We set $S_{*}^{\infty}\left(\Lambda_{y}\right)=\bigcup S_{*}^{m}\left(\Lambda_{y}\right)$ and $S_{*}^{-\infty}\left(\Lambda_{y}\right)=\bigcap S_{*}^{m}\left(\Lambda_{y}\right)$. Moreover, each element of $S_{*}^{m}()$ is called a symbol of order $m$. Here we put $*=0$ or nothing.

Associated to a symbol $p \in S^{m}\left(\Lambda_{x y}\right)$ is a pseudodifferential operator $P$ defined by

$$
P u(x)=\iint p(x, y, \eta) e^{i\langle x-y \mid \eta\rangle} u(y) d y d \eta
$$

where $u(y) \in C_{0}^{\infty}\left(U_{y}\right), \tilde{d} \eta=(2 \pi)^{-n} d \eta$. Hereafter, the integration as in (A.24) is always considered as the oscillatory integral and these notations for the above symbol classes also denote the corresponding classes of pseudodifferential operators.

Let $P$ be a pseudodifferential operator of order one with symbol $p \in S^{1}\left(\Lambda_{y}\right)$ and principal symbol $p_{1}(y, \eta)$. By regarding $p_{1}(y, \eta)$ as an element of $S_{0}^{1}\left({ }^{0} T^{*} U\right)$, we assume $\left({ }^{*} 1\right)$ and $\left({ }^{*} 2\right)$ for $p_{1}(y, \eta)$. Notice that $\left({ }^{*} 1\right)$ and $\left({ }^{*} 2\right)$ are open conditions for perturbations of the Hamiltonian flows. Then, by combining Theorem A.1 and the arguments in [H2, Lemma 2, 6.16], we get the following:

Proposition A.4. Assume $\left({ }^{*} 1\right)-\left({ }^{*} 2\right)$ for $P$ with $p_{1}(y, \eta) \in S_{0}^{1}\left({ }^{o} T^{*} U\right), U=U_{y}$. Let $z_{0}=\left(0, \eta_{0}\right)$ be a point of ${ }^{o} T^{*} U_{x}$ such that $p_{1}\left(z_{0}\right)=0$ and let $I$ be an open interval such that $\gamma: I \rightarrow{ }^{o} T^{*} U_{y}$ is a bicharacteristic strip satisfying $z_{0}=\gamma(0)$, then,

(i) there exist conic neighborhoods $\bar{D}_{z_{0}}$ and $\bar{D}_{\left(0, e_{n}\right)}$ of $\gamma(I)$ and $(t, 0, \ldots$, $\left.0, e_{n}\right) \quad(t \in I)$, and a canonical transformations $\chi: \bar{D}_{\left(0, e_{n}\right)} \rightarrow \bar{D}_{z_{0}}$ of positively 
homogeneous degree one such that

(ia) $\chi^{*} p_{1}(y, \eta)=\xi^{1}$

(ib) $\chi\left(x^{1}, 0, \ldots, 0, e_{n}\right)=\gamma\left(x^{1}\right)$.

(ii) There exist $k_{4} \in \mathbf{Z}_{+}, \delta_{4}>0$ such that for any given $s_{4} \in \mathbf{Z}_{+}$, the following properties hold: for $\left\|\tilde{p}_{1}-p_{1}\right\|_{(1,0), k_{4}}<\delta_{4}$, there exist a point $z_{0}\left(; \tilde{p}_{1}\right)=$ $\left(0, \eta_{0}\left(; \tilde{p}_{1}\right)\right)$, and a canonical transformation $\chi\left(; \tilde{p}_{1}\right): \bar{D}_{\left(0, e_{n}\right)} \rightarrow \bar{D}_{z_{0}}$ such that

(iia) $\tilde{p}_{1}\left(z_{0}\left(; \tilde{p}_{1}\right)\right)=0$ and a bicharacteristic strip $\gamma\left(; \tilde{p}_{1}\right): I \rightarrow{ }^{o} T^{*} U_{y}$ satisfying $\gamma(0)=z_{0}\left(; \tilde{p}_{1}\right)$ is contained in $\bar{D}_{z_{0}}$;

(iib) $\left(\chi\left(; \tilde{p}_{1}\right)^{*} \tilde{p}_{1}\right)(x, \xi)=\xi^{1}$;

(iic) $\chi\left(x^{1}, 0, \ldots, 0, e_{n}\right)=\gamma\left(x^{1}\right)$.

(iii) $z_{0}\left(; \tilde{p}_{1}\right)$ and $\chi\left(; \tilde{p}_{1}\right)$ satisfy

$$
\begin{aligned}
\left|z_{0}\left(; \tilde{p}_{1}\right)-z_{0}\right| & \leq C_{4}\left\|\tilde{p}_{1}-p_{1}\right\|_{(1,0), k_{4}}, \\
\left|\chi\left(; \tilde{p}_{1}\right)-\chi\left(; p_{1}\right)\right|_{\left.s_{4}, \bar{D}_{\left(0, e_{n}\right.}\right)} & \leq C_{4}\left\|\tilde{p}_{1}-p_{1}\right\|_{(1,0), s_{4}+k_{4}} .
\end{aligned}
$$

(A.III) Microlocal normal form of a real principal type operator. Using the canonical transformation given in Theorem A1, we give a normal form of a real principal type operator. The method is essentially a routine reworking of [E and $\mathrm{DH}]$.

Let $P$ (resp. $\widetilde{P}$ ) be a pseudodifferential operator of order one defined on an open set $U$ with symbol $p(y, \eta)$ (resp. $\tilde{p}(y, \eta))$. We may assume that the support of $p$ (resp. $\tilde{p}$ ) is contained in $T^{*} U$. We assume we are in the setup of Proposition A.4 and use the notation in (A.I). Moreover, we assume $\left({ }^{*} 1\right)-\left({ }^{*} 3\right)$ for $p_{1} \in S_{0}^{1}\left({ }^{o} T^{*} U\right)$.Then, by the openness of the conditions $\left({ }^{*} 1\right)-\left({ }^{*} 3\right)$, any $\tilde{p}_{1}$ sufficiently close to $p_{1}$ also satisfies $\left({ }^{*} 1\right)-\left({ }^{*} 3\right)$, after possibly reducing the size of $U$.

First, we construct a nondegenerate phase function associated to $\chi$ given in Proposition A.4. Using local coordinates $(x, \xi)$ in $\bar{D}_{\left(0, e_{n}\right)}$ and $(y, \eta)$ in $\bar{D}_{z_{0}}$ respectively, we express the canonical transformations $\chi$ by $\chi(x, \xi)=(y, \eta)$.

Let $z_{0}=\left(y_{0}, \eta_{0}\right) \in{ }^{o} T^{*} U$ and assume $y_{0}=0$. Let $V_{0}$ be the tangent space of the fiber through a point $z_{0}$ in $T_{z_{0}} T^{*} \mathbf{R}^{n} . V_{0}$ is a Lagrangian subspace. Put $V_{t}=\left(\exp t H_{p_{1}}\right)_{*} V_{0}$, where $\exp t H_{p_{1}}$ denotes the 1-parameter flow of the Hamiltonian vector field $H_{p_{1}}$. Then, $V_{t}$ can be identified with a subspace of $T_{z_{0}} T^{*} \mathbf{R}^{n}$. Set $\left(\chi^{-1}\right)_{*}\left(V_{0}\right)=V_{0}^{\prime}$ and $\left(\chi^{-1}\right)_{*}\left(V_{t}\right)=V_{t}^{\prime}$, so $V_{t}^{\prime}=\left(\exp t H_{\xi^{1}}\right)_{*} V_{0}^{\prime}$. By $\left({ }^{*} 3\right)$, there exists a Lagrangian subspace $\lambda^{\perp}$ of $T_{\left(0, e_{n}\right)} T^{*} U_{x}$ which is transversal to the tangent space of the fiber at $\left(0, e_{n}\right)$ and to $V_{t}^{\prime}$. Now, by the transversality of $\lambda^{\perp}$ and the tangent space of the fiber at $\left(0, e_{n}\right)$, we can write $\lambda^{\perp}$ as

$$
\lambda^{\perp}=\left\{\left(x_{0}, \xi_{0}, \delta x, \delta \xi\right) \mid \delta \xi^{i}=\sum A_{i j} \delta x^{j}\right\}
$$


where $A_{i j}$ is a symmetric matrix (cf. [D, Theorem 3.4.7]). Hence, by the coordinate change

$$
x_{1}^{\prime}=x_{1}, \ldots, x_{n-1}^{\prime}=x_{n-1}, x_{n}^{\prime}=x_{n}-(1 / 2) \sum A_{i j} x^{i} x^{j},
$$

$\lambda^{\perp}$ becomes the tangent space to the manifold $\xi=e_{n}$. For simplicity of notation, we denote the new coordinates $x^{\prime}$ again by $x$. In the coordinates $\left(x^{1}, \ldots, x^{n}\right)$, we consider the canonical relation

$$
C=\left\{(x, \xi, y, \eta) \mid(x, \xi)=\chi(y, \eta),(y, \eta) \in \bar{D}_{z_{0}},(x, \xi) \in \bar{D}_{\left(0, e_{n}\right)}\right\} .
$$

Since $\left(\chi^{-1}\right)_{*}\left(V_{t}\right)$ is transversal to the tangent space of the manifold $\xi=e_{n}$, the mapping

$$
(x, \xi, y, \eta) \in C \rightarrow(y, \xi)
$$

has injective differential at

$$
\left(t, 0, \ldots, 0, e_{n}, \exp t H_{p_{1}}\left(z_{0}\right)\right),
$$

whenever $\exp t H_{p_{1}}\left(z_{0}\right) \in \bar{D}_{z_{0}}$ (cf. [D, Lemma 3.7.4], and [H2, Proposition 25.3.3]). Thus, there exists a phase function $S(y, \xi)$ such that $C$ can be parametrized locally by $\left\{\left(y, \partial_{y} S(y, \xi), \partial_{\xi} S(y, \xi), \xi\right)\right\}$ and $S(y, \xi)$ is nondegenerate, i.e., it satisfies $\operatorname{det} \partial_{y} \partial_{\xi} S(y, \xi) \neq 0$. Furthermore, by taking sufficiently small conic neighborhoods, we may assume that this discussion still holds for the conic neighborhood chosen in Proposition A.4.

Sincerely the transversality condition is an open condition, the coordinates above can be still used for any small perturbation of $p_{1}$. So, for any $\tilde{p}_{1}$ sufficiently close to $p_{1}$, we take $\bar{D}_{\left(0, e_{n}\right)}, \bar{D}_{z_{0}}$ sufficiently small so that the mapping (A.27) associated to $\tilde{p}_{1}$ becomes a diffeomorphism onto a conic open set $\bar{C}$ which contains $\left(0, e_{n}\right)$.

Proposition A.5. In the above situation,

(i) there exists a phase function $S(; \chi)=S(y, \xi ; \chi) \in C^{\infty}(\bar{C})$ such that

(ia) $S(y, \xi ; \chi)$ is positively homogeneous of degree one with respect to $\xi$;

(ib) $S(y, \xi ; \chi)$ is nondegenerate, i.e.,

$$
\operatorname{det} \partial_{\xi} \partial_{y} S(y, \xi ; \chi) \neq 0 \quad \text { in } \bar{C} ;
$$

(ic) $x$ and $\eta$ satisfy

$$
x=\left(\partial_{\xi} S(; \chi)\right)(y, \xi), \quad \eta=\left(\partial_{y} S(; \chi)\right)(y, \xi) \quad \text { in } \bar{C} .
$$

(ii) There exist $k_{5} \in \mathbf{Z}_{+}$and $\delta_{5}>0$ such that for any $s_{5} \in \mathbf{Z}_{+}$, the following properties hold: for $\left\|\tilde{p}_{1}-p_{1}\right\|_{(1,0), k_{5}}<\delta_{5}$, there exists a phase function $S\left(y, \xi ; \chi\left(; \tilde{p}_{1}\right)\right) \in C^{\infty}(\bar{C})$ such that

(iia) $S\left(y, \xi ; \chi\left(; \tilde{p}_{1}\right)\right)$ satisfies the properties in (i) with $\chi=\chi\left(; \tilde{p}_{1}\right)$;

(iib) $S\left(x, \eta ; \chi\left(; \tilde{p}_{1}\right)\right)$ satisfies

$$
\| S\left(y, \xi ; \chi\left(; \tilde{p}_{1}\right)-S\left(y, \xi ; \chi\left(; p_{1}\right)\right)\left\|_{(1,0), s_{s}, \bar{C}} \leq C_{5}\right\| \tilde{p}_{1}-p_{1} \|_{(1,0), s_{s}+k_{s}}\right.
$$


for some positive constant $C_{5}$, where the norm is the restriction to $\bar{C}$ of (A.1) considered on $(y, \xi)$-space.

Let $U_{x}$ and $U_{y}$ be fixed relatively compact open neighborhoods of $x=0$ and $y=0$, respectively. Later on, we will take $U_{x}, U_{y}$ sufficiently small. We define $\Lambda_{x y}=U_{x} \times U_{y} \times\left(\mathbf{R}^{n}-\{0\}\right), \Lambda_{x y}=U_{y} \times U_{x} \times\left(\mathbf{R}^{n}-\{0\}\right), \Lambda_{y y}=U_{y} \times$ $U_{y} \times\left(\mathbf{R}^{n}-\{0\}\right)$, and $\Lambda_{x x}=U_{x} \times U_{x} \times\left(\mathbf{R}^{n}-\{0\}\right)$ respectively. Associated to the canonical transformation $\left.\chi: \bar{D}_{\left(0, e_{n}\right.}\right) \rightarrow \bar{D}_{z_{0}}$ with the generating phase function $S(y, \xi)$ on $C$ and a symbol $a(y, x, \xi) \in S^{m}\left(\Lambda_{y x}\right)$ with $\operatorname{supp}_{y, \xi} a(x, y, \eta)=$ $\{(y, \xi) \mid(y, x, \xi) \in \operatorname{supp} a$ for some $x\}$ contained properly in $\bar{C}$, we define a (microlocal) Fourier-integral operator order $m$ by the oscillatory integral

$$
(F(a, \chi) f)(y)=\iint a(y, x, \xi) e^{i(S(y, \xi ; \chi)-\langle x \mid \xi\rangle)} f(x) d x d \eta
$$

for any $f \in C_{0}^{\infty}\left(U_{x}\right)$. Now, we recall some product formulas for pseudodifferential and Fourier-integral operators [DH, H, K, OMY1-2 and OMYK7]. In the following, we fix an integer $n_{0}$ sufficiently large.

Proposition A.6. (i) Let $a=a(y, x, \xi) \in S^{m}\left(\Lambda_{x y}\right)$ and $b=b\left(x^{\prime}, x, \xi\right) \in$ $S^{m^{\prime}}\left(\Lambda_{x x}\right)$. Assume that $\operatorname{supp}_{y, \xi} a \subset \bar{C}$. There exist

$$
\begin{aligned}
& A(; a, b, \chi)=A(y, x, \xi ; a, b, \chi) \in S^{m+m^{\prime}}\left(\Lambda_{y x}\right), \\
& B(; a, b, \chi)=B(y, x, \xi ; a, b, \chi) \in S^{-n_{0}}\left(\Lambda_{y x}\right)
\end{aligned}
$$

and

such that

$$
B_{-\infty}(a, b, \chi)=B_{-\infty}(y, x, \xi ; a, b, \chi) \in S^{-n_{0}}\left(\Lambda_{y x}\right)
$$

$$
\begin{aligned}
F(a, \chi) P(b)= & F(A(; a, b, \chi), \chi)+F(B(; a, b, \chi), \chi) \\
& +P\left(B_{-\infty}(; a, b, \chi)\right)
\end{aligned}
$$

where

(ia) the symbol $A(; a, b, \chi)$ of (A.32) satisfies $\operatorname{supp}_{y, \xi} A \subset \bar{C}$;

(ib) the symbol $B(; a, b, \chi)$ of (A.32) satisfies $\operatorname{supp}_{x, \xi} B \subset \bar{C}$;

(ic) the principal symbol $A_{m+m^{\prime}}(; a, b, \chi)$ of $A(; a, b, \chi)$ is given by

(A.33) $A_{m+m^{\prime}}(y, x, \xi ; a, b, \chi)=a_{m}\left(y, \partial_{x} S(y, \xi ; \chi), \xi\right) b_{m^{\prime}}\left(\partial_{\xi} S(y, \xi ; \chi), x, \xi\right)$.

(ii) Similarly, given $a^{\prime}=a^{\prime}(y, x, \xi) \in S^{m}\left(\Lambda_{y x}\right)$ and $b^{\prime}=b^{\prime}\left(y^{\prime}, y, \eta\right) \in$ $S^{m^{\prime}}\left(\Lambda_{y y}\right)$, $\operatorname{supp}_{y, \xi} a^{\prime} \subset \bar{C}$, there exist $A^{\prime}\left(; a^{\prime}, b^{\prime}, \chi\right)=A^{\prime}\left(y, x, \xi ; a^{\prime}, b^{\prime}, \chi\right) \in$ $S^{m+m^{\prime}}\left(\Lambda_{x y}\right), \quad B^{\prime}\left(; a^{\prime}, b^{\prime}, \chi\right)=B^{\prime}\left(y, x, \xi ; a^{\prime}, b^{\prime}, \chi\right) \in S^{-n_{0}}\left(\Lambda_{y x}\right)$, and $B_{-\infty}^{\prime}\left(; a^{\prime}, b^{\prime}, \chi\right)=B_{-\infty}^{\prime}\left(y, x, \xi ; a^{\prime}, b^{\prime}, \chi\right)=S^{-n_{0}}\left(\Lambda_{y x}\right)$ such that

$$
\begin{aligned}
P\left(b^{\prime}\right) F\left(a^{\prime}, \chi\right)= & F\left(A^{\prime}\left(; a^{\prime}, b^{\prime}, \chi\right), \chi\right)+F\left(B^{\prime}\left(; a^{\prime}, b^{\prime}, \chi\right), \chi\right) \\
& +P\left(B_{-\infty}^{\prime}\left(; a^{\prime}, b^{\prime}, \chi\right)\right),
\end{aligned}
$$


where

(iia) $\operatorname{supp}_{y, \xi} A^{\prime} \subset \bar{C}$;

(iib) $\operatorname{supp}_{y, \xi} B^{\prime} \subset \bar{C}$;

(iic) the principal symbol $A_{m+m^{\prime}}^{\prime}$ of $A^{\prime}(; a, b, \chi)$ is given by

$$
A_{m+m^{\prime}}^{\prime}(y, x, \xi ; a, b, \chi)=b_{m^{\prime}}^{\prime}\left(y, y, \partial_{y} S(y, \xi ; \chi)\right) a_{m}^{\prime}(y, x, \xi)
$$

(iii) There exist $k_{6} \in \mathbf{Z}_{+}, \delta_{6}>0$ such that, for any $m_{6} \in \mathbf{Z}_{+}$, the following estimates hold:

$$
\begin{aligned}
& \|A(; \tilde{a}, \tilde{b}, \tilde{\chi})-A(; a, b, \chi)\|_{m+m^{\prime}, m_{6}} \\
& \leq C_{6}\left\{|\tilde{\chi}-\chi|_{m_{6}+k_{6}, \bar{D}_{\left(0, c_{n}\right)}}+\|\tilde{a}-a\|_{m, m_{6}+k_{6}}+\|\tilde{b}-b\|_{m^{\prime}, m_{6}+k_{6}}\right\}, \\
& \|B(; \tilde{a}, \tilde{b}, \tilde{\chi})-B(; a, b, \chi)\|_{-n_{0}, m_{6}} \\
& \leq C_{6}\left\{|\tilde{\chi}-\chi|_{m_{6}+k_{6}, \bar{D}_{\left(0, c_{n}\right)}}+\left\|\tilde{a}^{\prime}-a\right\|_{m, m_{6}+k_{6}}+\left\|\tilde{b}^{\prime}-b^{\prime}\right\|_{m^{\prime}, m_{6}+k_{6}}\right\}, \\
& \left\|B_{-\infty}(; \tilde{a}, \tilde{b}, \tilde{\chi})-B_{-\infty}(; a, b, \chi)\right\|_{-n_{0}, m_{6}} \\
& \leq C_{6}\left\{|\tilde{\chi}-\chi|_{m_{6}+k_{6}, \bar{D}_{\left(0, e_{n}\right)}}+\left\|\tilde{a}^{\prime}-a\right\|_{m, m_{6}+k_{6}}+\left\|\tilde{b}^{\prime}-b\right\|_{m^{\prime}, m_{6}+k_{6}}\right\}, \\
& \left\|A^{\prime}\left(; \tilde{a}^{\prime}, \tilde{b}^{\prime}, \tilde{\chi}\right)-A^{\prime}\left(; a^{\prime}, b^{\prime}, \chi\right)\right\|_{m+m^{\prime}, m_{6}} \\
& \leq C_{6}\left\{|\tilde{\chi}-\chi|_{m_{6}+k_{6}, \bar{D}_{\left(0, e_{n}\right)}}+\left\|\tilde{a}^{\prime}-a^{\prime}\right\|_{m, m_{6}+k_{6}}+\left\|\tilde{b}^{\prime}-b^{\prime}\right\|_{m^{\prime}, m_{6}+k_{6}}\right\}, \\
& \left\|\boldsymbol{B}^{\prime}\left(; \tilde{a}^{\prime}, \tilde{b}^{\prime}, \tilde{\chi}\right)-\boldsymbol{B}^{\prime}\left(; a^{\prime}, b^{\prime}, \chi\right)\right\|_{-n_{0}, m_{6}} \\
& \leq C_{6}\left\{|\tilde{\chi}-\chi|_{m_{6}+k_{6}, \bar{D}_{\left(0, c_{n}\right)}}+\left\|\tilde{a}^{\prime}-a^{\prime}\right\|_{m, m_{6}+k_{6}}+\left\|\tilde{b}^{\prime}-b^{\prime}\right\|_{m^{\prime}, m_{6}+k_{6}}\right\}, \\
& \left\|B_{-\infty}^{\prime}\left(; \tilde{a}^{\prime}, \tilde{b}^{\prime}, \tilde{\chi}\right)-B_{-\infty}^{\prime}\left(; a^{\prime}, b^{\prime}, \chi\right)\right\|_{-n_{0}, m_{6}} \\
& \leq C_{6}\left\{|\tilde{\chi}-\chi|_{m_{6}+k_{6}, \bar{D}_{\left(0, e_{n}\right)}}+\left\|\tilde{a}^{\prime}-a^{\prime}\right\|_{m, m_{6}+k_{6}}+\left\|\tilde{b}^{\prime}-b^{\prime}\right\|_{m^{\prime}, m_{6}+k_{6}}\right\},
\end{aligned}
$$

if $|\tilde{\chi}-\chi|_{k_{6}, \bar{D}_{\left(0, c_{n}\right)}}<\delta_{6},\|\tilde{a}-a\|_{m, k_{6}}<\delta_{6},\|\tilde{b}-b\|_{m, k_{6}}<\delta_{6},\left\|\tilde{a}^{\prime}-a^{\prime}\right\|_{m, k_{6}}<\delta_{6}$, $\left\|\tilde{a}^{\prime}-a^{\prime}\right\|_{m, k_{6}}<\delta_{6}$, and $a, b, \ldots$ satisfy the assumptions of (i) and (ii).

Proof. Composing (A.24) and (A.31), we have

$$
\begin{aligned}
& (F(a ; \chi) P(b)) f(y) \\
& \quad=\iint e^{i\left(S(y, \xi)-\langle x \mid \xi\rangle+\left\langle x-x^{\prime} \mid \eta^{\prime}\right\rangle\right.} a(y, x, \xi) b\left(x, x^{\prime}, \eta\right) f\left(x^{\prime}\right) d x^{\prime} \tilde{d} \eta d x \tilde{d} \xi .
\end{aligned}
$$

Given a positive constant $M>0$, let $\nu(\xi) \in C_{0}^{\infty}\left(\mathbf{R}^{n}\right)$ be a cutoff function satisfying

$$
\nu(\xi) \equiv 1 \quad \text { on }|\xi| \leq \frac{1}{2} M, \quad \nu(\xi) \equiv 0 \quad \text { on }|\xi| \geq M
$$

Divide (A.37) into two parts:

$$
F(a, \chi) P(b) f(y)=\left(I^{\infty}(; a, b, \chi) f(y)+I_{0}(; a, b, \chi) f(y),\right.
$$


where

$$
\begin{aligned}
& I^{\infty}(; a, b, \chi) f(y) \\
& =\int \cdots \int e^{i\left(S(y, \xi)-\langle x \mid \xi\rangle+\left\langle x-x^{\prime} \mid \eta\right\rangle\right)} a(y, x, \xi) b\left(x, x^{\prime}, \eta\right) \nu(\xi) f\left(x^{\prime}\right) d x^{\prime} \tilde{d} \eta d x \tilde{d} \xi \\
& =\iint e^{i\left\langle y-x^{\prime} \mid \eta\right\rangle}\left\{\iint e^{i[S(y, \xi)-\langle x \mid \xi-\eta\rangle-\langle y \mid \eta\rangle]} a(y, x, \xi)\right. \\
& \left.\times \nu(\xi) b\left(x, x^{\prime}, \eta\right) d x \tilde{d} \xi\right\} f\left(x^{\prime}\right) d x^{\prime} \tilde{d} \eta
\end{aligned}
$$

and

$$
\begin{aligned}
& I_{0}(; a, b, \chi) f(y)=\iint e^{i\left(S(y, \xi)-\langle x \mid \xi\rangle+\left\langle x-x^{\prime} \mid \eta\right\rangle\right)} \\
& \quad \times a(y, x, \xi) b\left(x, x^{\prime}, \eta\right)(1-\nu(\xi)) f\left(x^{\prime}\right) d x^{\prime} \tilde{d} \eta d x \tilde{d} \xi .
\end{aligned}
$$

We easily see that $I^{\infty}(; a, b, \chi)$ can be absorbed into the third term on the righthand side of (A.32). Now we consider $I_{0}(; a, b, \chi)$. Choose positive constants $0<C_{1}<C_{2}$, and $C^{\infty}$ cutoff function $\psi(\xi, \eta)$ of positively homogeneous of degree zero, satisfying

(A.40) $\psi(\xi, \eta) \equiv 1$ on $\left\{|\xi-\eta| \leq C_{1}|\eta|\right\} \quad$ and $\operatorname{supp} \psi \subset\left\{|\xi-\eta| \leq C_{2}|\eta|\right\}$.

We divide $I_{0}$ into two parts:

$$
I_{0}(; a, b, \chi) f(y)=I_{0}^{\infty}(; a, b, \chi) f(y)+I_{0}^{\prime}(; a, b, \chi) f(y),
$$

where

$$
\begin{gathered}
I_{0}^{\infty} f(y)=\iint e^{i\left(S(y, \xi)-\langle x \mid \xi\rangle+\left\langle x-x^{\prime} \mid \eta\right\rangle\right)} a(y, x, \xi) b\left(x, x^{\prime}, \eta\right)(1-\nu(\xi)) \\
\times(1-\psi(\xi, \eta)) f\left(x^{\prime}\right) d x^{\prime} \tilde{d} \eta d x \tilde{d} \xi \\
=\iint e^{i\left\langle y-x^{\prime} \mid \eta\right\rangle}\left\{\iint e^{i[S(y, \xi)-\langle x \mid \xi-\eta\rangle-\langle y \mid \eta\rangle]} a(y, x, \xi) b\left(x, x^{\prime}, \eta\right)\right. \\
\times(1-\nu(\xi))(1-\psi(\xi, \eta)) d x, \tilde{d} \xi\} f\left(x^{\prime}\right) d x^{\prime} \tilde{d} \eta
\end{gathered}
$$

and

$$
I_{0}^{\prime} f(y)=\iint e^{i\left(S(y, \eta)-\left\langle x^{\prime} \mid \eta\right\rangle\right.} I_{0}^{\prime \prime}\left(y, x^{\prime}, \eta\right) f\left(x^{\prime}\right) d x^{\prime} \tilde{d} \eta
$$

where

$$
\begin{aligned}
& I_{0}^{\prime \prime}\left(y, x^{\prime}, \eta\right) \\
& \quad=\int e^{i[S(y, \xi)-S(y, \eta)-\langle x \mid \xi-\eta\rangle]} a(y, x, \xi) b\left(x, x^{\prime}, \eta\right)(1-\nu(\xi)) \psi(\xi, \eta) d x \tilde{d} \xi .
\end{aligned}
$$

Notice that

$$
\begin{gathered}
\left|\partial_{x}\left\{S(y, \xi)-\langle x \mid \xi\rangle+\left\langle x-x^{\prime} \mid \eta\right\rangle\right\}\right|=|\xi-\eta| \geq C_{1}|\eta|, \\
|\xi| \leq\left(1+C_{1}^{-1}\right)|\xi-\eta| \text { on } \operatorname{supp}(1-\psi) .
\end{gathered}
$$


Thus, using integration by parts, we see that $I_{0}^{\infty}$ can also be absorbed into the third term of (A.32). Since

$$
S(y, \xi)-S(y, \eta)-\langle y \mid \xi-\eta\rangle=\left\langle\int_{0}^{1} \partial_{\xi} S(y, \eta+\theta(\xi-\eta) d \theta-x|\xi-\eta\rangle,\right.
$$

we put

$$
\eta^{\prime \prime}=\xi-\eta, \quad y^{\prime \prime}=\int_{0}^{1} \partial_{\xi} S(y, \eta+\theta(\xi-\eta) ; \chi) d \theta-x .
$$

By Taylor's theorem, we have

$$
\begin{aligned}
& I_{0}^{\prime \prime}=\iint e^{i\left\langle y^{\prime \prime} \mid \eta^{\prime \prime}\right\rangle}(1-\nu(\xi)) \psi\left(\eta^{\prime \prime}+\eta, \eta\right) \\
& \quad \times a\left(y, \nabla_{\xi} S\left(y, \eta^{\prime \prime}+\eta, \eta\right)-y^{\prime \prime}, \eta^{\prime \prime}+\eta\right) \\
& \quad \times b\left(\nabla_{\xi} S\left(y, \eta^{\prime \prime}+\eta, \eta\right)-y^{\prime \prime}, x^{\prime}, \eta\right) d y^{\prime \prime} \tilde{d} \eta^{\prime \prime} \\
& =F(A(; a, b, \chi), \chi)+F(B(; a, b, \chi), \chi)
\end{aligned}
$$

where

$$
\nabla_{\xi} S(y, \xi, \eta)=\int_{0}^{1} \partial_{\xi} S(y, \eta+\theta(\xi-\eta) d \theta),
$$

and $A(; a, b, \chi)$ and $B(a ; b, \chi)$ satisfy Proposition A.6(i). In fact, since $\left|\eta^{\prime \prime}\right| \leq$ $C_{1}|\eta|$ on $\operatorname{supp} \psi\left(\eta^{\prime \prime}+\eta, \eta\right)$, we get

$$
\left|\eta^{\prime \prime}+\eta\right| \geq|\eta|-\left|\eta^{\prime \prime}\right| \geq\left(1-C_{2}\right)|\eta| \quad \text { on } \operatorname{supp} \psi\left(\eta^{\prime \prime}+\eta, \eta\right) \text {. }
$$

Also, since $\left|\eta^{\prime \prime}+\eta\right| \geq C_{2}^{-1}\left(1-C_{2}\right)\left|\eta^{\prime \prime}\right|$ on $\operatorname{supp} \psi\left(\eta^{\prime \prime}+\eta, \eta\right)$, the principal symbol of $A$ can be easily obtained from the Taylor series for the amplitude of (A.22) around $\eta^{\prime \prime}=0$ and integration by parts with respect to $y^{\prime \prime}$. The property (ia) is clear from (ic), and (ib) follows from $\left|\eta^{\prime \prime}\right| \leq C_{2}|\eta|$ and $\left(1-C_{2}\right)|\eta| \leq$ $\left|\eta^{\prime \prime}+\eta\right| \leq\left(1+C_{2}\right)|\eta|$ on supp $\psi\left(\eta^{\prime \prime}+\eta, \eta\right)$ provided that $C_{2}$ is taken sufficiently small. The principal symbol of $A$ is easily obtained.

Now we shall prove (ii). Computing $P\left(; b^{\prime}\right) F\left(; a^{\prime}, \chi\right)$ directly, we have (A.44)

$$
\begin{aligned}
& P\left(; b^{\prime}\right) F\left(; a^{\prime}, \chi\right) f(y) \\
& \quad=\int \cdots \int e^{i\left[\left\langle y-y^{\prime} \mid \eta\right\rangle+S\left(y^{\prime}, \xi\right)-\langle x \mid \xi\rangle\right]} b^{\prime}\left(y, y^{\prime}, \eta\right) a^{\prime}\left(y^{\prime}, x, \xi\right) f(x) d x \tilde{d} \xi d y^{\prime} \tilde{d} \eta,
\end{aligned}
$$

for $f \in C_{0}^{\infty}\left(U_{x}\right)$. Using the cutoff function $\nu(\xi) \in C_{0}^{\infty}(\mathbf{R})$ given in (A.38), we divide (A.44) into two parts:

$$
P\left(; b^{\prime}\right) F\left(; a^{\prime}, \chi\right)=J^{\infty}\left(; a^{\prime}, b^{\prime}, \chi\right)+J_{0}\left(; a^{\prime}, b^{\prime}, \chi\right)
$$

where

$$
\begin{aligned}
J^{\infty}\left(; a^{\prime}, b^{\prime}, \chi\right)=\int \cdots \int e^{i\left(\left\langle y-y^{\prime} \mid \eta\right\rangle+S\left(y^{\prime}, \xi\right)-\langle x \mid \xi\rangle\right)} & \\
& \times a^{\prime}\left(y^{\prime}, x, \xi\right) b^{\prime}\left(y, y^{\prime}, \eta\right) \nu(\xi) f(x) d x \tilde{d} \xi d y^{\prime} \tilde{d} \eta
\end{aligned}
$$


and

$$
\begin{aligned}
J_{0}\left(; a^{\prime}, b^{\prime}, \chi\right)=\int \cdots \int e^{i\left(\left\langle y-y^{\prime} \mid \eta\right\rangle+S\left(y^{\prime}, \xi\right)-\langle x \mid \xi\rangle\right)} & \\
& \times a^{\prime}\left(y^{\prime}, x, \xi\right) b^{\prime}\left(y^{\prime}, y^{\prime}, \eta\right)(1-\nu(\xi)) f(x) d x \tilde{d} \xi d y^{\prime} \tilde{d} \eta .
\end{aligned}
$$

Rewrite $J^{\infty}\left(; a^{\prime}, b^{\prime}, \chi\right)$ as follows:

$$
J^{\infty}\left(; a^{\prime}, b^{\prime}, \chi\right)=\iint e^{i\langle y-x \mid \eta\rangle} B^{\prime \prime \infty}(y, x, \eta) f(x) d x \tilde{d} \eta,
$$

where

$$
\begin{aligned}
& B^{\prime \prime \infty}\left(y, x, \eta ; a^{\prime}, b^{\prime}, \chi\right) \\
& \quad=\iint e^{-i\left\langle y^{\prime}-x \mid \eta\right\rangle}\left\{e^{i\left(S\left(y^{\prime}, \xi\right)-\langle x \mid \xi\rangle\right)} b^{\prime}\left(y, y^{\prime}, \eta\right)\right\} a^{\prime}\left(y^{\prime}, x, \xi^{\prime}\right) \nu(\xi) d y^{\prime} \tilde{d} \xi .
\end{aligned}
$$

By a direct computation, we see that $B^{\prime \prime \infty}(; a, b, \chi)$ can be absorbed into the third term of (A.34).

Next we consider $J_{0}\left(; a^{\prime}, b^{\prime}, \chi\right)$. Notice that there exists a positive constant $C>1$ such that

$$
\begin{aligned}
C|\xi| \geq\left|\int_{0}^{1} \partial_{y} S\left(y+\theta\left(y^{\prime}-y\right), \xi\right) d \theta\right| & \geq C^{-1}|\xi| \\
& \text { for } y, y^{\prime}, \xi \text { such that }(y, \xi),\left(y^{\prime}, \xi\right) \in \bar{C} .
\end{aligned}
$$

We choose positive constants $0<C_{1}<C_{2}$ with $C_{1}$ is small enough and $C_{2}$ is large enough so that

$$
\begin{aligned}
& \left\{(\xi, \eta)\left|C_{1}\right| \xi|<| \eta\left|<C_{2}\right| \xi \mid\right\} \\
& \quad \supset\left\{\left(\xi, \int_{0}^{1} \partial_{y} S\left(y+\theta\left(y^{\prime}-y\right), \xi\right) d \theta\right) ;(y, \xi),\left(y^{\prime}, \xi\right) \in \bar{C}\right\} .
\end{aligned}
$$

We take a smooth function $\psi(\xi, \eta)$ on $\mathbf{R}^{n} \times \mathbf{R}^{n}-\{(0,0)\}$ of homogeneous degree 0 such that $\operatorname{supp} \psi \subset\left\{(\xi, \eta)\left|(1 / 2) C_{1}\right| \xi|<| \eta\left|<2 C_{2}\right| \xi \mid\right\}$, and

$$
\psi(\xi, \eta) \equiv 1 \quad \text { on }\left\{C_{1}|\xi|<|\eta|<C_{2}|\xi|\right\}
$$

Moreover, we divide $J_{0}\left(; a^{\prime}, b^{\prime}, \chi\right)$ into two parts:

$$
J_{0}\left(; a^{\prime}, b^{\prime}, \chi\right) f(y)=J_{0}^{\infty}\left(; a^{\prime}, b^{\prime}, \chi\right) f(y)+J_{0}^{\infty}\left(; a^{\prime}, b^{\prime}, \chi\right) f(y),
$$

where

$$
\begin{aligned}
& J_{0}^{\infty}\left(; a^{\prime}, b^{\prime}, \chi\right) f(y)=\int \cdots \int e^{i\left[\left\langle y-y^{\prime} \mid \eta\right\rangle+S\left(y^{\prime}, \xi\right)-\langle x \mid \xi\rangle\right]}(1-\nu(\xi))(1-\psi(\xi, \eta)) \\
&=\iint e^{i\langle y-x \mid \xi\rangle}\left\{\iint e^{i\left[-\left\langle y^{\prime}-y \mid \eta\right\rangle+S\left(y^{\prime}, \xi\right)-\langle y \mid \xi\rangle\right]} b^{\prime}\left(y, y^{\prime}, \xi\right)\right. \\
&\left.\cdot a^{\prime}\left(y^{\prime}, x, \xi\right)(1-\nu(\xi))(1-\psi(\xi, \eta)) d y^{\prime} \tilde{d} \eta\right\} f(x) d x \tilde{d} \xi
\end{aligned}
$$


and

$$
\begin{aligned}
J_{0}^{\prime}\left(; a^{\prime}, b^{\prime}, \chi\right) f(y)=\int \cdots \int e^{i\left[\left\langle y-y^{\prime} \mid \eta\right\rangle+S\left(y^{\prime}, \xi\right)-\langle x \mid \xi\rangle\right]}(1-\nu(\xi)) \psi(\xi, \eta) & \\
& \times b^{\prime}\left(y, y^{\prime}, \eta\right) a^{\prime}\left(y^{\prime}, x, \xi\right) f(x) d x \tilde{d} \xi d y^{\prime} \tilde{d} \eta
\end{aligned}
$$

By the definition of $\psi$, we get

$$
\left|\partial_{y^{\prime}}\left[-\left\langle y^{\prime}-y \mid \eta\right\rangle+S\left(y^{\prime}, \xi\right)-\langle y \mid \xi\rangle\right]\right| \geq C_{3}(|\xi|+|\eta|) \quad \text { on } \operatorname{supp}(1-\psi)
$$

for some $C_{3}$. Then, using the operator

$$
L=\left\{\left|\partial_{y^{\prime}}\left[-\left\langle y^{\prime}-y \mid \eta\right\rangle+S\left(y^{\prime}, \xi\right)-\langle y \mid \xi\rangle\right]\right|\right\}^{-2} \partial_{y^{\prime}}\left[-\left\langle y^{\prime}-y \mid \eta\right\rangle+S\left(y^{\prime}, \xi\right)-\langle y \mid \xi\rangle\right] \cdot \frac{1}{i} \partial_{y^{\prime}}
$$

we easily see that $J_{0}^{\infty}$ can be absorbed into the third term in (A.34). Changing the variables $\left(x^{\prime}, \eta\right)$ to

$$
Y=y^{\prime}-y, \quad \zeta=\int_{0}^{1} \partial_{y} S\left(y^{\prime}+\theta\left(y^{\prime}-y\right), \xi\right) d \theta-\eta,
$$

we rewrite $J_{0}^{\prime}\left(; a^{\prime}, b^{\prime}, \chi\right)$ as

$$
J_{0}^{\prime}\left(; a^{\prime}, b^{\prime}, \chi\right) f(y)=\iint e^{i(S(y, \xi)-\langle x \mid \xi\rangle)} B^{\prime \prime}(y, x, \xi) f(x) d x \tilde{d} \xi
$$

for

$$
\begin{aligned}
& B^{\prime \prime}\left(y, x, \xi ; a^{\prime}, b^{\prime}, \chi\right) \\
& =\iint e^{i\langle Y \mid \zeta\rangle} \psi\left(\xi, \nabla_{y} S\left(y, y^{\prime}, \xi\right)-\zeta\right) \psi\left(\xi, \nabla_{y} S\left(y, y^{\prime}, \xi\right)-\zeta\right) \\
& \times\left(1-\nu\left(\nabla_{y} S\left(y, y^{\prime}, \xi\right)-\zeta\right) b^{\prime}\left(y, Y+y, \nabla_{y} S\left(y, y^{\prime}, \xi\right)-\zeta\right)\right. \\
& \quad \times a^{\prime}(Y+y, x, \xi) d Y \tilde{d} \zeta
\end{aligned}
$$

where we put $\nabla_{x} S\left(y, y^{\prime}, \xi\right)=\int_{0}^{1} \partial_{y} S\left(y+\theta\left(y^{\prime}-y\right), \eta\right) d \theta$. Here we may assume $|Y|=\left|y^{\prime}-y\right|$ is sufficiently small; if not we absorb the part for rest of $Y$ into the third term in (A.34). By a Taylor expansion around $\zeta=0$, integration by parts and the definition of $\psi$, we easily obtain (iia), (iic). Property (iib) is easily verified by taking $\left(y^{\prime}-y\right)$ sufficiently small.

Finally, we trivially check property (iii). In fact, the only ambiguity in the previous computation is the choice of cutoff functions. These functions can be fixed if $\tilde{\chi}$ is sufficiently close to $\chi$. Thus, the continuity of the symbols of (A.32) and (A.34) in $a, b, a^{\prime}, B^{\prime}$ and $\chi$ is easily obtained (cf. [H1, K and OMY2]).

We define the adjoint type operators of (A.32) by

$$
\left(F^{+}(d, \chi) g(x)=\iint d(x, y, \eta) e^{i(\langle x \mid \eta\rangle-S(y, \eta ; \chi))} g(y) d y \tilde{d} \eta,\right.
$$

where $d(x, y, \eta) \in S^{m}\left(\Lambda_{x y}\right)$ and $\operatorname{supp}_{y, \eta} d \subset \bar{C}$. 
Proposition A.7. (i) Let $d=d\left(y, y^{\prime}, \eta^{\prime}\right) \in S^{m}\left(\Lambda_{y y}\right)$ and $e=e(x, y, \eta) \in$ $S^{m^{\prime}}\left(\Lambda_{x y}\right)$ with $\operatorname{supp}_{y^{\prime}, \eta^{\prime}} d\left(y, y^{\prime}, \eta^{\prime}\right) \subset \bar{C}$. Then, there exist symbols

$$
\begin{gathered}
C(; e, d, \chi)=C(x, y, \eta ; e, d, \chi) \in S^{m+m^{\prime}}\left(\Lambda_{x y}\right) \\
D(; e, d, \chi)=D(x, y, \eta ; e, d, \chi) \in S^{-n_{0}}\left(\Lambda_{x y}\right)
\end{gathered}
$$

and

$$
D_{-\infty}(; e, d, \chi)=D_{-\infty}(x, y, \eta ; e, d, \chi) \in S^{-n_{0}}\left(\Lambda_{x y}\right)
$$

such that

$$
\begin{aligned}
P(e) F^{+}(d, \chi)= & F^{+}(C(; e, d, \chi) ; \chi) \\
& +F^{+}(D(; e, d, \chi) ; \chi)+P\left(D_{-\infty}(e, d, \chi)\right)
\end{aligned}
$$

where

(ia) $\operatorname{supp}_{y, \eta} C(; e, d, \chi) \subset \bar{C}$;

(ib) $\operatorname{supp}_{y, \eta} D(; e, d, \chi) \subset \bar{C}$;

(ic) the principal symbol of $C(; e, d, \chi)$ is given by

$$
C_{m+m^{\prime}}(x, y, \eta)=e\left(x, \nabla_{\eta} S(y, \eta), \eta\right) d\left(\nabla_{\eta} S(y, \eta), y, \eta\right)
$$

(ii) Let $d^{\prime}=d^{\prime}(x, y, \eta) \in S^{m}\left(\Lambda_{x y}\right)$ and $e^{\prime}=e^{\prime}\left(y, y^{\prime}, \eta^{\prime}\right) \in S^{m^{\prime}}\left(\Lambda_{y y}\right)$ with $\operatorname{supp}_{y, \eta} d^{\prime}(x, y, \eta) \subset \bar{C}$. Then there exist symbols

$$
\begin{aligned}
& C^{\prime}\left(; e^{\prime}, d^{\prime}, \chi\right)=C^{\prime}\left(x, y, \eta ; e^{\prime}, d^{\prime}, \chi\right) \in S^{m+m^{\prime}}\left(\Lambda_{x y}\right) \\
& D^{\prime}\left(; e^{\prime}, d^{\prime}, \chi\right)=D^{\prime}\left(x, y, \eta ; e^{\prime}, d^{\prime}, \chi\right) \in S^{-n_{0}}\left(\Lambda_{x y}\right)
\end{aligned}
$$

and

$$
D_{-\infty}^{\prime}\left(; e^{\prime}, d^{\prime}, \chi\right)=D_{-\infty}^{\prime}\left(x, y, \eta ; e^{\prime}, d^{\prime}, \chi\right) \in S^{-n_{0}}\left(\Lambda_{x y}\right)
$$

such that

$$
\begin{aligned}
F^{+}\left(d^{\prime}, \chi\right) P\left(e^{\prime}\right)= & F^{+}\left(C^{\prime}\left(; e^{\prime}, d^{\prime}, \chi\right) ; \chi\right) \\
& +F^{+}\left(D^{\prime}\left(; a^{\prime}, b^{\prime}, \chi\right) ; \chi\right)+P\left(D_{-\infty}^{\prime}\left(; e^{\prime}, d^{\prime}, \chi\right)\right)
\end{aligned}
$$

where

(iia) $\operatorname{supp}_{y, \eta} C^{\prime}\left(; e^{\prime}, d^{\prime}, \chi\right) \subset \bar{C}$;

(iib) $\operatorname{supp}_{y, \eta} D^{\prime}\left(; e^{\prime}, d^{\prime}, \chi\right) \subset \bar{C}$;

(iic) the principal symbol of $C^{\prime}\left(; e^{\prime}, d^{\prime}, \chi\right)$ is given by

$$
C_{m+m^{\prime}}^{\prime}(x, y, \eta)=d^{\prime}(x, y, \eta) e^{\prime}\left(y, y, \nabla_{y} S(y, \eta)\right) .
$$


(iii) There exists $k_{7} \in \mathbf{Z}_{+}$, and $\delta_{7}>0$, such that for any $m_{7} \in \mathbf{Z}_{+}$, the following estimates hold:

$$
\begin{aligned}
& \|C(; \tilde{e}, \tilde{d}, \tilde{\chi})-C(; e, d, \chi)\|_{m+m^{\prime}, m_{7}} \\
& \leq C_{7}\left\{|\tilde{\chi}-\chi|_{m_{7}+k_{7}, \bar{D}\left(0, e_{n}\right)}+\|\tilde{d}-d\|_{m, m_{7}+k_{7}}+\|\tilde{e}-e\|_{m^{\prime}, m_{7}+k_{7}}\right\}, \\
& \|D(; \tilde{e}, \tilde{d}, \tilde{\chi})-D(; \boldsymbol{e}, d, \chi)\|_{-n_{0}, m_{7}} \\
& \leq C_{7}\left\{|\tilde{\chi}-\chi|_{m_{7}+k_{7}, \bar{D}_{\left(0 . c_{n}\right)}}+\left\|\tilde{d}^{\prime}-d^{\prime}\right\|_{m, m_{7}+k_{7}}+\|\tilde{e}-e\|_{m^{\prime}, m_{7}+k_{7}}\right\}, \\
& \left\|D_{-\infty}(; \tilde{e}, \tilde{d}, \tilde{\chi})-D_{-\infty}(; e, d, \chi)\right\|_{-n_{0}, m_{7}} \\
& \leq C_{7}\left\{|\tilde{\chi}-\chi|_{m_{7}+k_{7}, \bar{D}_{\left(0, e_{n}\right)}}+\|\tilde{d}-d\|_{m, m_{7}+k_{7}}+\|\tilde{e}-e\|_{m^{\prime}, m_{7}+k_{7}}\right\}, \\
& \left\|C^{\prime}(; \tilde{e}, \tilde{d}, \tilde{\chi})-C^{\prime}(; \boldsymbol{e}, d, \chi)\right\|_{m+m^{\prime}, m_{7}} \\
& \leq C_{7}\left\{|\tilde{\chi}-\chi|_{m_{7}+k_{7}, \bar{D}_{\left.10, r_{n}\right)}}+\|\tilde{d}-d\|_{m, m_{7}+k_{7}}+\|\tilde{e}-e\|_{m^{\prime}, m_{7}+k_{7}}\right\}, \\
& \left\|D^{\prime}(; \tilde{e}, \tilde{d}, \tilde{\chi})-D^{\prime}(; e, d, \chi)\right\|_{-n_{0}, m_{7}} \\
& \leq C_{7}\left\{|\tilde{\chi}-\chi|_{m_{7}+k_{7}, \bar{D}_{\left(0 . c_{n}\right)}}+\|\tilde{d}-d\|_{m, m_{7}+k_{7}}+\|\tilde{e}-e\|_{m^{\prime}, m_{7}+k_{7}}\right\}, \\
& \left\|D_{-\infty}^{\prime}\left(; \tilde{e}^{\prime}, \tilde{d}^{\prime}, \tilde{\chi}\right)-D_{-\infty}^{\prime}\left(; e^{\prime}, d^{\prime}, \chi\right)\right\|_{-n_{0}, m_{7}} \\
& \leq C_{7}\left\{|\tilde{\chi}-\chi|_{m_{7}+k_{7}, \bar{D}_{\left(0 . c_{n}\right)}}+\left\|\tilde{d}^{\prime}-d^{\prime}\right\|_{m, m_{7}+k_{7}}+\left\|\tilde{e}^{\prime}-e^{\prime}\right\|_{m^{\prime}, m_{7}+k_{7}}\right\},
\end{aligned}
$$

if $\|\tilde{\chi}-\chi\|_{k_{7}, \bar{D}_{\left(0 . c_{n}\right)}}<\delta_{7},\|\tilde{d}-d\|_{m, k_{7}}<\delta_{7},\left\|\tilde{d}^{\prime}-d^{\prime}\right\|_{m, k_{7}}<\delta_{7},\|\tilde{e}-e\|_{m^{\prime}, k_{7}}<\delta_{7}$ and $\left\|\tilde{e}^{\prime}-e^{\prime}\right\|_{m^{\prime}, k_{7}}<\delta_{7}$ and $\tilde{d}, \ldots, \tilde{e}^{\prime}$ are as in (i) or (ii).

Proof. Since the proof is almost the same as in Proposition A.6, we only indicate the cutoff functions used in the proof of (i). As for the proof of (ii), see the proof of Proposition A.6(ii), which is essentially the same.

Using the cutoff function $\nu\left(\eta^{\prime}\right)$ defined by (A.38), we make the following decomposition:

(A.56)

$$
\begin{aligned}
& \left(P(e) F^{+}(d, \chi) f\right)(x) \\
& \quad=\int \cdots \int e^{i\left[\langle x-y \mid \eta\rangle+\left\langle y \mid \eta^{\prime}\right\rangle-S\left(y^{\prime}, \eta^{\prime}\right)\right]} e(x, y, \eta) d\left(y, y^{\prime}, \eta^{\prime}\right) f\left(y^{\prime}\right) d y^{\prime} \tilde{d} \eta^{\prime} d y \tilde{d} \eta \\
& \quad=I^{* \infty}(; e, d, \chi) f(x)+I_{0}^{*}(; e, d, \chi) f(x),
\end{aligned}
$$

where

$$
\begin{gathered}
I^{* \infty}(; e, d, \chi) f(x)=\int \cdots \int e^{i\left[\langle x-y \mid \eta\rangle+\left\langle y \mid \eta^{\prime}\right\rangle-S\left(y^{\prime}, \eta^{\prime}\right)\right]} \nu\left(\eta^{\prime}\right) \\
\cdot e(x, y, \eta) d\left(y, y^{\prime}, \eta^{\prime}\right) f\left(y^{\prime}\right) d y^{\prime} \tilde{d} \eta^{\prime} d y \tilde{d} \eta, \\
I_{0}^{*}(; e, d, \chi) f(x)=\int \cdots \int e^{i\left[\langle x-y \mid \eta\rangle+\left\langle y \mid \eta^{\prime}\right\rangle-S\left(y^{\prime}, \eta^{\prime}\right)\right]}\left(1-\nu\left(\eta^{\prime}\right)\right) \\
\cdot e(x, y, \eta) d\left(y, y^{\prime}, \eta^{\prime}\right) f\left(y^{\prime}\right) d y^{\prime} \tilde{d} \eta^{\prime} d y \tilde{d} \eta .
\end{gathered}
$$

Moreover, taking the cutoff function $\psi\left(\eta^{\prime}, \eta\right)$ defined by (A.40), we make the following decomposition:

$$
I_{0}^{*}(; e, d, \chi) f(x)=I_{0}^{* \infty}(; e, d, \chi) f(x)+I_{0}^{* \prime}(; e, d, \chi) f(x),
$$


where

$$
\begin{aligned}
& I_{0}^{* \infty}(; e, d, \chi) f(x) \\
& =\int \cdots \int e^{i\left[\langle x-y \mid \eta\rangle+\left\langle y \mid \eta^{\prime}\right\rangle-S\left(y^{\prime}, \eta\right)\right]}\left(1-\psi\left(\eta^{\prime}, \eta\right)\right)\left(1-\nu\left(\eta^{\prime}\right)\right) \\
& \cdot e(x, y, \eta) d\left(y, y^{\prime}, \eta^{\prime}\right) f\left(y^{\prime}\right) d y^{\prime} \tilde{d} \eta^{\prime} d y \tilde{d} \eta, \\
& I_{0}^{* \prime}(; e, d, \chi) f(x) \\
& \quad=\int \cdots \int e^{i\left[\langle x-y \mid \eta\rangle+\left\langle y \mid \eta^{\prime}\right\rangle-S\left(y^{\prime}, \eta^{\prime}\right)\right]} \psi\left(\eta^{\prime}, \eta\right)\left(1-\nu\left(\eta^{\prime}\right)\right) \\
& \cdot e(x, y, \eta) d\left(y, y^{\prime}, \eta^{\prime}\right) f\left(y^{\prime}\right) d y^{\prime} \tilde{d} \eta^{\prime} d y \tilde{d} \eta .
\end{aligned}
$$

Proposition A.8. Assume $U_{x}$ is convex. (i) Let $b^{1}=b^{1}(x, \eta) \in S^{m}\left(\Lambda_{x y}\right)$ and $b^{2}=b^{2}(x, \eta) \in S^{m \prime}\left(\Lambda_{x y}\right), \operatorname{supp}_{x, \eta} b^{i} \subset \bar{C}(i=1,2)$. Then there exist symbols $E\left(; b^{1}, b^{2}, \chi\right) \in S^{m+m^{\prime}}\left(\Lambda_{x x}\right)$ and $E_{-\infty}\left(; b^{1}, b^{2}, \chi\right) \in S^{-n_{0}}\left(\Lambda_{x x}\right)$, such that

$$
F\left(b^{2}, \chi\right) F^{+}\left(b^{1}, \chi\right)=P\left(E\left(; b^{2}, b^{1}, \chi\right)\right)+P\left(E_{-\infty}\left(; b^{2}, b^{1}, \chi\right)\right),
$$

and the principal symbol of $E$ is given by

$$
E_{m+m^{\prime}}\left(x, x^{\prime}, \xi\right)=b^{2}(x, \eta(x, \xi)) b^{1}\left(x^{\prime}, \eta(x, \xi)\right) \mid \operatorname{det}\left(\left.\partial_{x} \partial_{\eta} S(x, \eta(x, \xi))\right|^{-1},\right.
$$

where $\eta(x, \xi)$ is the function implicitly defined by $\xi=\partial_{x} S(x, \eta)$.

(ii) There exists $k_{8} \in \mathbf{Z}_{+}, \delta_{8}>0$ such that for any $m_{8} \in \mathbf{Z}_{+}$, the following estimates hold:

$$
\begin{aligned}
& \left\|E\left(; \tilde{b}^{2}, \tilde{b}^{1}, \tilde{\chi}\right)-E\left(; b^{2}, b^{1}, \chi\right)\right\|_{m+m^{\prime}, m_{8}} \\
& \quad \leq C_{8}\left\{|\tilde{\chi}-\chi|_{m_{8}+k_{8}, \bar{D}_{\left(0, c_{n}\right)}}+\left\|\tilde{b}^{1}-b^{1}\right\|_{m, m_{8}+k_{8}}+\left\|\tilde{b}^{2}-b^{2}\right\|_{m^{\prime}, m_{8}+k_{8}}\right\}, \\
& \left\|E_{-\infty}\left(; \tilde{b}^{2}, \tilde{b}^{1}, \tilde{\chi}\right)-E_{-\infty}\left(; b^{2}, b^{1}, \chi\right)\right\|_{-n_{0}, m_{8}} \\
& \quad \leq C_{8}\left\{|\tilde{\chi}-\chi|_{m_{8}+k_{8}, \bar{D}_{\left(0, c_{n}\right)}}+\left\|\tilde{b}^{1}-b^{1}\right\|_{m, m_{8}+k_{8}}+\left\|\tilde{b}^{2}-b^{2}\right\|_{m^{\prime}, m_{8}+k_{8}}\right\}
\end{aligned}
$$

for some constant $C_{8}>0$, if $|\tilde{\chi}-\chi|_{k_{8}, \bar{D}_{\left(0 . e_{n}\right)}}<\delta_{8},\left\|\tilde{b}^{1}-b^{1}\right\|_{m, k_{8}}<\delta_{8}$, and $\left\|\tilde{b}^{2}-b^{2}\right\|_{m^{\prime}, k_{8}}<\delta_{8}$ and $\tilde{b}^{i}, i=1,2$, are as in (i).

Proof. By direct computation, we have

$$
\begin{aligned}
& \left(F\left(b^{2}, \chi\right) F^{+}\left(b^{1}, \chi\right) f\right)(x) \\
& \quad=\iint e^{i\left(S(x, \eta)-S\left(x^{\prime}, \eta\right)\right)} b^{2}(x, \eta) b^{1}\left(x^{\prime}, \eta\right) f\left(x^{\prime}\right) d x^{\prime} \tilde{d} \eta .
\end{aligned}
$$

Using the cutoff function $\nu(\eta)$ defined by (A.38), we make the following decomposition:

(A.62) $\left(F\left(b^{2}, \chi\right) F^{+}\left(b^{1}, \chi\right) f\right)(x)=H^{\infty}\left(; b^{1}, b^{2}, \chi\right) f(x)+H_{0}\left(; b^{1}, b^{2}, \chi\right) f(x)$, where

$$
H^{\infty}\left(; b^{1}, b^{2}, \chi\right) f(x)=\iint e^{i\left(S(x, \eta)-S\left(x^{\prime}, \eta\right)\right)} \nu(\eta) b^{2}(x, \eta) b^{1}\left(x^{\prime}, \eta\right) f\left(x^{\prime}\right) d x^{\prime} \tilde{d} \eta,
$$




$$
\begin{aligned}
H_{0}\left(; b^{1}, b^{2}, \chi\right) & f(x) \\
= & \iint e^{i\left(S(x, \eta)-S\left(x^{\prime}, \eta\right)\right)}(1-\nu(\eta)) b^{2}(x, \eta) b^{1}\left(x^{\prime}, \eta\right) f\left(x^{\prime}\right) d x^{\prime} \tilde{d} \eta
\end{aligned}
$$

It is easy to see that $H^{\infty}$ can be absorbed into the second term of (A.58). Since $S(x, \eta)-S\left(x^{\prime}, \eta\right)=\left(x-x^{\prime}\right) \nabla_{x} S\left(x, x^{\prime}, \eta\right)$, where

$$
\begin{gathered}
\nabla_{x} S\left(x, x^{\prime}, \eta\right)=\int_{0}^{1} \partial_{x} S\left(x^{\prime}+\theta\left(x-x^{\prime}\right), \eta\right) d \theta \\
H_{0}\left(; b^{1}, b^{2}, \chi\right) f(x) \\
=\iint e^{i\left\langle x-x^{\prime} \mid \nabla_{x} S\left(x, x^{\prime}, \eta\right)\right\rangle}(1-\nu(\eta)) b^{2}(x, \eta) b^{1}\left(x^{\prime}, \eta\right) f\left(x^{\prime}\right) d x^{\prime} \tilde{d} \eta
\end{gathered}
$$

Making the change of variables $\xi=\nabla_{x} S\left(x, x^{\prime}, \eta\right)$, we get

$$
\begin{array}{r}
H_{0}\left(; b^{1}, b^{2}, \chi\right) f(x)=\iint e^{i\left\langle x-x^{\prime} \mid \xi\right\rangle}\left(1-\nu\left(\eta\left(x, x^{\prime}, \xi\right)\right) b^{2}\left(x, \eta\left(x, x^{\prime}, \xi\right)\right)\right. \\
\cdot b^{1}\left(x^{\prime}, \eta\left(x, x^{\prime}, \xi\right)\right)\left|\frac{D(\eta)}{D(\xi)}\right| f\left(x^{\prime}\right) d x^{\prime} \tilde{d} \eta
\end{array}
$$

where $\eta\left(x, x^{\prime}, \xi\right)$ is the function implicitly defined by $\xi=\nabla_{x} S\left(x, x^{\prime}, \eta\right)$. Since the phase function $S(x, \eta)$ on $\bar{C}$ is defined by $\chi$, the existence of $\eta\left(x, x^{\prime}, \xi\right)$ is assured by the convexity of $U_{x}$. Now, by the Taylor expansion around $\eta=0$ and integration by parts, we easily see (i). Also, just as before, the property (ii) may be trivially verified.

We need to consider invertible pseudodifferential operators, of order 0 on $U=U_{x}=U_{y}$, using an easy computation as in [K, OMY1 and OMYK7]. Although [OMY1 and OMYK7] work on a compact Riemannian manifold $N$, here we only consider the simple case where $N$ is an open neighborhood of 0 with a flat metric.

Proposition A.9. (i) Let $P(a)$ be a pseudodifferential operator defined by (A.24) with symbol $a=a\left(x, x^{\prime}, \xi\right) \in S^{m}\left(\Lambda_{x x}\right)$. Then there exists $a^{\prime}(; a)=a^{\prime}(x, \xi) \in$ $S^{m}\left(\Lambda_{x}\right)$ such that

$$
P(a)=P\left(a^{\prime}(; a)\right)
$$

Let $c=c(x, \xi) \in S^{-m}\left(\Lambda_{x}\right)$. Assume that $P(c)$ is elliptic on $\Gamma_{\left(0, e_{n}\right)}\left(r_{9 x}, r_{9 \xi}\right)$. Then there exist symbols $g(; c) \in S^{-m}\left(\Lambda_{x}\right), h(; c) \in S^{-n_{0}}\left(\Lambda_{x}\right), q^{\prime}(; c) \in$ $S^{-m}\left(\Lambda_{x}\right)$ and $h^{\prime}(; c) \in S^{-n_{0}}\left(\Lambda_{x}\right)$ such that

$$
P(c) P(q(; c))-I=P(h(; c)), \quad P\left(q^{\prime}(; c)\right) P(c)-I=P\left(h^{\prime}(; c)\right) .
$$


(ii) Moreover, in (i) and (ii), there exist $k_{9} \in \mathbf{Z}_{+}, \delta_{9}>0$, such that for any positive $m_{9}$, the following estimates hold:

$$
\begin{gathered}
\left\|a^{\prime}(; \tilde{a})-a(; a)\right\|_{m, m_{9}} \leq C_{9}\|\tilde{a}-a\|_{m_{9}+k_{9}}, \\
\|q(; \tilde{c})-q(; c)\|_{-m, m_{9}} \leq C_{9}\|\tilde{c}-c\|_{-m, m_{9}+k_{9}}, \\
\|h(; \tilde{c})-h(; c)\|_{-n_{0}, m_{9}} \leq C_{9}\|\tilde{c}-c\|_{-m, m_{9}+k_{9}}, \\
\left\|q^{\prime}(; \tilde{c})-q^{\prime}(; c)^{\prime}\right\|_{-m, m_{9}} \leq C_{9}\|\tilde{c}-c\|_{-m, m_{9}+k_{9}}, \\
\left\|h^{\prime}(; \tilde{c})-h^{\prime}(; c)\right\|_{-n_{0}, m_{9}} \leq C_{9}\|\tilde{c}-c\|_{-m, m_{9}+k_{9}}
\end{gathered}
$$

for some positive constant $C_{9}$, if $\|\tilde{a}-a\|_{m, k_{9}}>\delta_{9},\|\tilde{c}-c\|_{-m, k_{9}}>\delta_{9}$.

Finally, we give a microlocal normal form of a real principal type operator as follows. Take open conic neighborhoods $D_{\left(0, e_{n}\right)}$ and $D_{z_{0}}$ of $\left(0, e_{n}\right)$ and $z_{0}$ satisfying $D_{\left(0, e_{n}\right)} \subset \bar{D}_{\left(0, e_{n}\right)}$ and $D_{z_{0}} \subset \bar{D}_{z_{0}}, \chi\left(D_{\left(0, e_{n}\right)}\right)=D_{z_{0}}$ respectively. Also, put $C^{\prime}=D_{\left(0, e_{n}\right)} \times D_{z_{0}}$ and denote by $\bar{C}^{\prime}$ the image of the mapping (A.27).

Theorem A.10. (i) Let $p(y, \eta) \in C^{\infty}\left({ }^{o} T^{*} \mathbf{R}^{n}\right)$ be a symbol of order 1 and assume $\left({ }^{*} 1\right)-\left({ }^{*} 3\right)$. Given $z_{0}=\left(0, \eta_{0}\right) \in{ }^{o} T^{*} U_{y}$, let $\bar{\Gamma}_{z_{0}}$ be a conic open neighborhood and $\chi(; p)$ be a canonical transformation positively homogeneous of degree one defined on $\bar{D}_{\left(0, e_{n}\right)}$ given in Proposition A.4 such that $\chi\left(0, e_{n}\right)=z_{0}$, $\chi\left(\bar{D}_{\left(0, e_{n}\right)}\right)=\bar{\Gamma}_{z_{0}}$. Then there exist symbols $b^{1}=b^{1}(y, x, \xi ; \chi) \in S^{0}\left(\Lambda_{y x}\right)$ and $b^{2}=b^{2}\left(x, x^{\prime}, \xi^{\prime} ; \chi\right) \in S^{0}\left(\Lambda_{x x}\right)$ with $\operatorname{supp}_{y, \xi} b^{1}, \operatorname{supp}_{x^{\prime}, \xi^{\prime}} b^{2} \subset \bar{C}$ and symbols $k_{1}(; p) \in S^{-n_{0}}\left(\Lambda_{y y}\right)$ and $k_{2}(; p) \in S^{-n_{0}}\left(\Lambda_{x x}\right)$ such that

$$
\begin{gathered}
F\left(b^{1}(; p)\right) F^{+}\left(b^{2}(; p)\right)=I+P\left(k^{1}(; p)\right) \quad \text { on } D_{z_{0}}, \\
P(; p) F\left(b^{1}(; p) ; \chi\right)-F\left(b^{1}(; p) ; \chi\right) D_{x^{1}}=P\left(k^{2}(; p)\right)
\end{gathered}
$$

(iii) There exist $k_{10} \in \mathbf{Z}_{+}, \delta_{10}>0$ such that, for any $m_{10} \in \mathbf{Z}_{+}$, the following properties hold: for $\tilde{p}(y, \eta) \in S^{1}\left({ }^{o} T^{*} U_{y}\right)$ satisfying $\|\tilde{p}-p\|_{k_{10}}<\delta_{10}$, we have

$$
\begin{gathered}
\left\|b^{i}(; \tilde{p})-b^{i}(; p)\right\|_{0, m_{10}} \leq C_{10}\|\tilde{p}-p\|_{1, m_{10}+k_{10}} \text { for } i=1,2 ; \\
|\chi(; \tilde{p})-\chi(; p)|_{m_{10}, \bar{D}_{\left(0 . c_{n}\right)} \leq C_{10}\|\tilde{p}-p\|_{1, m_{10}+k_{10}}} ; \\
\left\|k^{i}(; p)-k^{i}(; p)\right\|_{-n_{0}, k_{10}} \leq C_{10}\|\tilde{p}-p\|_{1, m_{10}+k_{10}} \text { for } i=1,2
\end{gathered}
$$

for some positive constant $C_{10}$.

Proof. Let $F(a, \chi)$ and $F^{+}(a, \chi)$ be Fourier integral operators with symbol $a=a(y, \xi) \in S^{0}\left(\Lambda_{y x}\right)$ satisfying

$$
\operatorname{supp}_{y, \xi} a \subset \bar{C}, \quad a \equiv 1 \text { on } C .
$$

Then we have

$$
F(a, \chi) F^{+}(a, \chi) \equiv I, \quad \text { on } D_{z_{0}}
$$


and

$$
P F(a, \chi)-F(a, \chi) D_{1}=F(a, \chi) R
$$

where $D_{1}=(1 / i) \partial / \partial x^{1}$ and $R$ is of degree 0 . Now, by the following method we will drop $F(a, \chi) R$ up to order $-n_{0}$. To do this, we seek $A$ satisfying $A\left(D_{1}+R\right) A^{-1}=D_{1}$. Namely, we consider
(A.72)
$\left[A, D_{1}\right]+A R \equiv 0 \quad\left(\bmod S^{-n_{0}}\right)$.

This can be solved by successive approximations, writing $A$ as a sum $A=$ $\sum_{j=0}^{\infty} A_{j}$ and seek each $A_{j} \in S^{-j}\left(\Lambda_{x x}\right)$ such that $Q_{N} \equiv\left[\sum_{j=0}^{N} A_{j}, D_{1}\right]$ $+\left(\sum_{j=0}^{N} A_{j}\right) R \in S^{-N-1}\left(\lambda_{x x}\right)$. This only requires that each $A_{N}$ satisfies the equation

$$
i \frac{\partial A_{N}}{\partial x^{1}}+\sigma(R) A_{N}=-\sigma\left(Q_{N-1}\right),
$$

where $\sigma(\cdot)$ denotes the principal symbol. The above equations have solutions, homogeneous of degree $-N$ in the considered neighborhood, because the trajectory of $\partial / \partial x^{1}$ is contained in it. Thus there exists some amplitude $b^{1}$ satisfying (i). Moreover, by multiplying (A.70) by a suitable elliptic pseudodifferential operator on the right, we get (i). By the method above, the continuous dependence of the solution can be directly obtained by Proposition A.5 and Propositions A.8-A.11.

\section{APPENDIX B}

The purpose of this part of appendix is to prove Theorem 3.1.

Put

$$
\Psi=\Phi\left(u+u_{0}\right)-\Phi\left(u_{0}\right), \quad f=g-\Phi\left(u_{0}\right) .
$$

If $u \in C^{\infty}\left(\mathbf{R}^{n}, \mathbf{R}^{N}\right)$ is a solution of the equation

$$
\Psi(u)=f \text { in } U_{1},
$$

then $u+u_{0}$ will clearly be a solution of the (3.3).

Rewriting the conditions (3.1) and (3.2) in terms of $\Phi^{\prime}(u)$, we obtain that, for any $u\left(\|u\|_{\alpha} \leq \delta\right), s \geq d$ and $h \in H^{s}\left(\mathbf{R}^{n}\right)$,

$$
\Psi^{\prime}(u) v=h \text { in } U_{1},
$$

admits a solution $v \in H^{s-d}\left(\mathbf{R}^{n}\right)$ with the estimate

$$
\|v\|_{s-d} \leq C_{s}\left(\|h\|_{s}+\|u\|_{s}\|h\|_{d}\right),
$$

where $C_{s}$ is independent of $u$ and $h$. For future reference, we denote this $v$ by $\Psi^{\prime}(u)^{-1} h$.

In order to solve (B.1), we define functions $\left\{u_{n}\right\}$ by

$$
\left\{\begin{array}{l}
u_{1}=0, \\
u_{n+1}=u_{n}+s_{\theta_{n}} \rho_{n} \quad(n \geq 1),
\end{array}\right.
$$


where $s_{\theta}(\theta \geq 1)$ are the usual smoothing operators for the Banach scale $H^{s}\left(\mathbf{R}^{n}\right)$ $(s \in \mathbf{R}), \theta_{n}=\theta^{\tau^{n+n_{0}}}$ with $\tau=\frac{4}{3}$ and large enough $\theta>1, n_{0}$, and $\rho_{n}=$ $\Psi^{\prime}\left(u_{n}\right)^{-1} \varepsilon R g_{n}$ with

$$
g_{n}=f-\Psi\left(u_{n}\right)
$$

Here

$$
\varepsilon: H^{s}\left(U_{1}\right) \rightarrow H^{s}\left(\mathbf{R}^{n}\right), \quad R: H^{s}\left(\mathbf{R}^{n}\right) \rightarrow H^{s}\left(U_{1}\right)
$$

are the extension operator and the restriction operator, respectively.

First we prove the following inequalities $(\mathrm{i})_{j},(\mathrm{ii})_{j},(\mathrm{iii})_{j-1}(j \geq 1)$ by induction on $j$. Namely, there exists a sufficiently large integer $s_{0} \geq m$ and small $\eta(0<\eta<1)$ such that, for any $f\left(\|f\|_{s_{0}}^{0}<\eta\right)$ and nonnegative integer $j$,

(i) ${ }_{j}\left\|u_{j}\right\|_{\alpha} \leq \delta$;

(ii) $\left\|g_{j}\right\|_{\alpha}^{0} \leq M \theta_{j}^{-\mu}\|f\|_{s_{0}}^{0}$;

(iii) ${ }_{j}\left\|\rho_{j}\right\|_{\alpha} \leq M \theta_{j}^{-\alpha}\|f\|_{s_{0}}^{0}$.

are valid for some positive numbers $M, \mu, a$ independent of $f$ and $j$.

For $j=1$, the validity of these inequalities immediately follows by setting

$$
M=\theta_{1}^{\mu} .
$$

Assuming their validity for $j \leq n$, we shall next prove that these inequalities also hold for $j=n+1$.

Lemma B.1. Suppose

$$
\alpha \geq[n / 2]+m+1
$$

and $(\mathrm{i})_{n}$ is valid. Then

$$
\left\|g_{n}\right\|_{s}^{0} \leq C_{s}\left\{\left\|g_{1}\right\|_{s}^{0}+\left\|u_{n}\right\|_{s+m}^{0}\right\} \quad \text { for any } s \geq 0 .
$$

Here, $[n / 2]$ denotes the largest integer which is not greater than $n / 2$.

Proof. This follows from the Sobolev embedding theorem and the following lemma to

$$
g_{n}-g_{1}=-\Psi\left(u_{n}\right) \equiv-\Psi\left(x, \bar{D}^{m} u_{n}\right) .
$$

Lemma B.2 (Moser's Lemma). Let $\Omega$ be a domain in $\mathbf{R}^{n}$ whose boundary is a compact $C^{\infty}$ hypersurface and let $F(x, v) \in B^{\infty}\left(\Omega \times\left\{v \in \mathbf{R}^{l} ;|v| \leq v_{0}\right\}\right)$ with $F(x, 0)=0$. Then for any $s \geq 0$ and $v \in H^{s}(\Omega),\|v\|_{L^{\infty}(\Omega)} \leq v_{0}$, we have

$$
\|F(\cdot, v(\cdot))\| \leq C\left(s,|F|_{B^{s+1}}\right)\|v\|_{H^{s}(\Omega)},
$$

where $|F|_{B^{s+1}}$ denotes the maximum of the supnorms in $\Omega \times\left\{|v| \leq v_{0}\right\}$ of all derivatives of $F(x, v)$ up to order $s+1$.

Lemma B.3. Suppose

$$
\alpha \geq m+d
$$


and (i) ${ }_{j}$ is valid for $1 \leq j \leq n$. Then for any integer $s^{*} \geq m+d$, there exists $\theta>1$ such that

$$
\left\|u_{n+1}\right\|_{s} \leq \theta_{n+1}^{(m+d) /(\tau-1)+1}\left\|g_{1}\right\|_{s}^{0},
$$

for any $s\left(m+d \leq s \leq s^{*}\right)$.

Proof. For the rest of the proof of Theorem 3.1, we use a common symbol $K$ to denote any constant which depends only on $s, \delta$ and $\eta$. By (B.3), (B.4) and noting that $\left\|\varepsilon R g_{j}\right\|_{s} \leq K\left\|g_{i}\right\|_{s}^{0}$ for any $s \geq 0$, we have

$$
\left\|u_{i+1}\right\|_{s} \leq\left\|u_{i}\right\|_{s}+\left\|S_{\theta_{i}} \rho_{i}\right\|_{s} \leq K \theta_{i}^{m+d}\left(\left\|u_{i}\right\|_{s}+\|f\|_{s-m}^{0}\right)
$$

for any $i(1 \leq i \leq n)$. Using (B.12) recursively and taking $\theta$ large enough, we see that

$$
\left\|u_{n+1}\right\|_{s} \leq \theta_{n+1}^{(m+d) /(\tau-1)+1}\|f\|_{s}^{0}
$$

holds for any $s\left(m+d \leq s \leq s^{*}\right)$.

Now we assume $(\mathrm{i})_{j},(\mathrm{ii})_{j}(1 \leq j \leq n)$ and try to prove $(\mathrm{i})_{n+1},(\mathrm{ii})_{n+1}$,

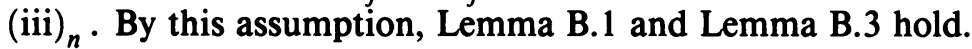

We first prove (ii) $)_{n+1}$. From (B.5),

$$
g_{n+1}-g_{n}=-\left(\Psi\left(u_{n+1}\right)-\Psi\left(u_{n}\right)\right) .
$$

Combine (B.14) with

$$
\begin{aligned}
& \Psi\left(u_{n+1}\right)-\Psi\left(u_{n}\right)-\Psi^{\prime}\left(u_{n}\right)\left(u_{n+1}-u_{n}\right) \\
& \quad=\int_{0}^{1} \theta \Psi^{\prime \prime}\left(\theta u_{n}+(1-\theta) u_{n+1}\right)\left(u_{n+1}-u_{n}\right) d \theta .
\end{aligned}
$$

Then we have

$$
g_{n=1}=g_{n}-\Psi^{\prime}\left(u_{n}\right)\left(u_{n+1}-u_{n}\right)+Q_{n}
$$

where

$$
Q_{n}=-\int_{0}^{1} \theta \cdot \Psi^{\prime \prime}\left(\theta u_{n}+(1-\theta) u_{n+1}\right)\left(u_{n+1}-u_{n}, u_{n+1}-u_{n}\right) d \theta
$$

Since

$$
\Psi^{\prime}\left(u_{n}\right) \rho_{n}=g_{n} \text { in } U_{1}
$$

(B.4) and (B.16) imply

$$
g_{n+1}=\Psi^{\prime}\left(u_{n}\right)\left(1-S_{\theta_{n}}\right) \rho_{n}+Q_{n} \text { in } U_{1} .
$$

From Lemma B.1, Lemma B.3 and (B.3), we have

$$
\begin{gathered}
\left\|\Psi^{\prime}\left(u_{n}\right)\left(1-S_{\theta_{n}}\right) \rho_{n}\right\|_{d}^{0} \leq K_{1} \theta_{n}^{(m+d) /(\tau-1)+2 m-s_{0}+2 d+1}\left\|g_{1}\right\|_{s_{0}}^{0}, \\
\left\|S_{\theta_{n}} \rho_{n}\right\|_{\alpha} \leq M K_{2} \theta_{n}^{\alpha-\mu}\left\|g_{1}\right\|_{\alpha}^{0}<M K_{2} \theta_{n}^{\alpha-\mu} \eta .
\end{gathered}
$$


Here and throughout the proof of Theorem 3.1, $K_{1}$ and $K_{2}$ denote constants depending only on $s_{0}$ and $\alpha$, respectively. If we take

$$
\mu>\alpha,
$$

a sufficiently large $\theta$ and a sufficiently small $\eta$ such that

$$
M K_{1} \theta_{n}^{\alpha-\mu} \eta \leq \delta
$$

the estimate

$$
\left\|\theta u_{n}+(1-\theta) u_{n+1}\right\| \leq \delta \quad(0 \leq \theta \leq 1)
$$

follows from (B.4), (B.21), (i) $)_{n}$ then,

$$
\left\|Q_{n}\right\|_{d}^{0} \leq K_{1} \theta_{n}^{2(\alpha-\mu)}\left\|g_{1}\right\|_{s_{0}}^{0}
$$

by (B.21), (B.24) and the well-known fact: for any integer $s \geq[n / 2]+1$, there exists $C_{s}>0$ such that

$$
\|v \cdot w\|_{H^{s}(\Omega)} \leq C_{s}\left(\|v\|_{L^{\infty}(\Omega)}\|w\|_{H^{s}(\Omega)}+\|w\|_{L^{\infty}(\Omega)}\|v\|_{H^{s}(\Omega)}\right)
$$

for any $v, w \in H^{s}(\Omega)$, for $\Omega$ as in Moser's Lemma. Therefore, from (B.19), (B.20), (B.25), we have

$$
\begin{gathered}
\left\|g_{n+1}\right\|_{d}^{0} \leq K_{1} \theta_{n}^{\beta}\left\|g_{1}\right\|_{s_{0}}^{0}, \\
\beta=\max \left(\frac{m+d}{\tau-1}+2 m-s_{0}+2 d+1,2(\alpha-\mu)\right) .
\end{gathered}
$$

These immediately yield (ii) $)_{n+1}$ if we take $\theta$ large enough and let $s_{0}, \mu$ satisfy

$$
s_{0} \geq 5(m+d)+2(\alpha-\mu)+1 \text {, }
$$

$$
\mu>3 \alpha
$$

so that $\beta>-\mu \tau$.

Secondly, we prove $(\mathrm{iii})_{n}$. By Lemma B.1, Lemma B.3, (B.3) and (ii) $)_{n}$,

$$
\begin{gathered}
\left\|\rho_{n}\right\|_{0} \leq K_{1} \theta_{n}^{-\mu}\left\|g_{1}\right\|_{s_{0}}^{0}, \\
\left\|\rho_{n}\right\|_{s_{0}-d-m} \leq K_{1} \theta_{n}^{(m+d) /(\tau-1)+1}\left\|g_{1}\right\|_{s_{0}}^{0}
\end{gathered}
$$

Suppose

$$
\alpha<s_{0}-m-d .
$$

Interpolating (B.30), (B.31), we have

$$
\left\|\rho_{n}\right\|_{\alpha} \leq K_{3} \theta_{n}^{\gamma}\left\|g_{1}\right\|_{s_{0}}^{0}
$$

with

$$
\gamma=\frac{-\mu\left(s_{0}-m-d-\alpha\right)+\alpha((m+d) /(\tau-1)+1)}{s_{0}-m-d} .
$$


As before, $K_{3}$ denotes a constant depending only on $\alpha, s_{0}$. Now, for $\mu$ satisfying

$$
\mu>\frac{(3 \alpha-2 a)(m+d)+2 a s_{0}+\alpha}{s_{0}-m-d-\alpha},
$$

which is equivalent to $\gamma<-2 a$, (iii) ${ }_{n}$ is valid for sufficiently large $\theta$.

Thirdly, we prove $(\mathrm{i})_{n+1}$. By (B.4) and (iii) $)_{j}(j \leq n)$, we have

$$
\left\|u_{n+1}\right\|_{\alpha} \leq \sum_{j=1}^{n}\left\|u_{j+1}-u_{j}\right\|_{\alpha} \leq K_{2} M\left\|g_{1}\right\|_{s_{0}}^{0} \sum_{j=1}^{n} \theta_{j}^{-\alpha} \leq K_{2} M \eta
$$

for sufficiently large $\theta$. Hence, $(\mathrm{i})_{n+1}$ is true provided that

$$
K_{2} M \eta \leq \delta \text {. }
$$

In the above argument, the required conditions are (B.7), (B.10), (B.22), (B.28), (B.32), (B.35) and (B.37). It is easy to check the existence of $\alpha, s_{0}, a$, $\mu, \eta$ satisfying these conditions. Thus we have proved $(\mathrm{i})_{j},(\mathrm{ii})_{j},(\mathrm{iii})_{j}(j \geq$ 1).

Next we show the existence of a solution $u$ of (B.1). By (B.36),

$$
\left\|u_{j+1}-u_{j}\right\|_{\alpha} \leq K_{2} M \theta_{j}^{-a}\left\|g_{1}\right\|_{s_{0}}^{0} \text {. }
$$

Thus $\left\{u_{j}\right\}$ is a Cauchy sequence in $H^{\alpha}\left(\mathbf{R}^{n}\right)$, because $\theta_{j}$ monotonically increases to infinity as $j \rightarrow \infty$. Consequently, there exists $u \in H^{\alpha}\left(\mathbf{R}^{n}\right)$ such that

$$
\lim _{j \rightarrow \infty} u_{j}=u \text { in } H^{\alpha}\left(\mathbf{R}_{n}\right)
$$

On the other hand, (ii) $)_{j}$ implies $g_{j} \rightarrow 0(j \rightarrow \infty)$ in $H^{d}\left(U_{1}\right)$. Hence, recalling (B.7) and letting $n$ tend to infinity in (B.5), we see that $u$ is a solution of (B.1).

Finally, we prove $u \in C^{\infty}\left(\mathbf{R}^{n}, \mathbf{R}^{N}\right)$ by following an idea due to [S]. It is enough to prove that $\left\{u_{j}\right\}$ converges in $H^{\alpha^{\prime}}\left(\mathbf{R}^{n}\right)$ for any $\alpha^{\prime}$. By the same arguments which led to (B.12) and (B.31), we have

$$
\begin{gathered}
\left\|u_{n+1}\right\|_{s} \leq K \theta_{n}^{m+d}\left(\left\|u_{n}\right\|_{s}+\left\|g_{1}\right\|_{s}^{0}\right), \\
\left\|\rho_{n}\right\|_{s-m-d} \leq K\left(\left\|g_{1}\right\|_{s-m}^{0}+\left\|u_{n}\right\|_{s}\right) \text { for any } s \geq m+d .
\end{gathered}
$$

Using (B.39) recursively, we have

$$
1+\left\|u_{n+1}\right\|_{s} \leq K \theta_{n+1}^{6(m+d)}
$$

Combining (B.40) and (B.41) ${ }_{n}$, we obtain

$$
\left\|\rho_{n}\right\|_{s-m-d} \leq K \theta_{n}^{6(m+d)}
$$

Now, suppose $s \geq m+d, \alpha^{\prime}<s-m-d$. The interpolation of (B.30) and (B.42) yields

$$
\left\|\rho_{n}\right\|_{\alpha^{\prime}} \leq K_{4} \theta_{n}^{\sigma}
$$




$$
\sigma=\frac{-\mu\left(s-m-d-\alpha^{\prime}\right)+6 \alpha^{\prime}(m+d)}{s-m-d},
$$

where $K_{4}$ is a constant depending only on $s_{0}, s, \delta$ and $\eta$. Clearly, by taking $s$ large enough in (B.43), (B.44), we have

$$
\left\|\rho_{n}\right\|_{\alpha^{\prime}} \leq K_{4} \theta_{n}^{-b} \text { for some } b>0 \text {. }
$$

As before, we can prove that $\left\{u_{j}\right\}$ is also a Cauchy sequence in $H^{\alpha^{\prime}}\left(\mathbf{R}^{n}\right)$. Since $\alpha^{\prime}$ can be taken arbitrarily large, we can conclude $u \in C^{\infty}\left(\mathbf{R}^{n}, \mathbf{R}^{N}\right)$ with the aid of Sobolev's embedding theorem. This completes the proof of Theorem 3.1.

\section{REFERENCES}

[BGY] R. Bryant, P. A. Griffiths and D. Yang, Characteristics and existence of isometric embeddings, Duke Math. J. 3 (1983), 893-994.

[D] J. J. Duistermaat, Fourier integral operators, Courant Institute of Math. Sci., New York Univ., 1973.

[DH] J. J. Duistermaat and L. Hörmander, Fourier integral operators. II, Acta Math. 128 (1972), 183-269.

[DY] D. DeTurck and D. Yang, Local existence of smooth metrics with prescribed curvature, Nonlinear Problems in Geometry, (D. DeTurck, ed.), Contemp. Math., vol. 51, Amer. Math. Soc., Providence, R.I., 1985, pp. 37-43.

[E] Ju. V. Egorov, Canonical transformations and pseudodifferential operators, Trans. Moscow Math. Soc. 24 (1971), 1-28.

[GY] J. B. Goodman and D. Yang, Local solvability of nonlinear partial differential equations of real principal type (to appear).

[H] L. Hörmander, Fourier integral operators. I, Acta Math. 127 (1971), 79-183.

[H1] _ , The analysis of linear partial differential operators IV, Grundlehren Math. Wiss., vol. 275, Springer-Verlag, 1985.

[J] H. Jacobowitz, Local isometric embeddings, Ann. of Math. Stud., no. 102, Princeton Univ. Press, 1982.

[K] H. Kumano-go, Pseudo-differential operators, MIT Press, Cambridge, Mass., 1981.

[L] C. S. Lin, The local isometric embedding $\mathbf{R}^{3}$ of two dimensional Riemannian manifolds with Gaussian curvature changing sign cleanly, Ph.D. dissertation, Courant Institute, 1983.

[M] S. Mizohata, The theory of partial differential equations, Cambridge Univ. Press, Cambridge, 1973.

[OMY1] H. Omori, Y. Maeda and A. Yoshioka, On regular Fréchet-Lie groups. I, Some differential geometrical expressions of Fourier-integral operators on a Riemannian manifold, Tokyo J. Math. 3 (1980), 353-390.

[OMY2] __, On regular Fréchet-Lie groups. II, Composition rules of Fourier-integral operators on a Riemannian manifold, Tokyo J. Math. 4 (1981), 221-253.

[OMYK3] H. Omori, Y. Maeda, A. Yoshioka and O. Kobayashi, On regular Fréchet-Lie groups. III, A second cohomology class related to the Lie algebra of pseudo-differential operators of order one, Tokyo J. Math. 4 (1981), 255-277.

[OMYK4] __, On regular Fréchet-Lie groups. IV, Definition and fundamental theorems, Tokyo J. Math. 5 (1982), 365-398.

[OMYK5] __, On regular Fréchet-Lie groups. V, Several basic properties, Tokyo J. Math. 6 (1983), 39-64.

[OMYK6] _, On regular Fréchet-Lie groups. VII, The group generated by pseudo-differential operators of negative order, Tokyo J. Math. 7 (1984), 315-336. 
[OMYK7] _ _, On regular Fréchet-Lie groups. VIII, Primordial operators and Fourier integral operators, Tokyo J. Math. 8 (1985), 1-47.

[S] M. F. Sergeraert, Une généralisation du théorème des fonctions implicites de Nash, C. R. Acad. Sci. Paris 270A (1970), 861-863.

[T] J. F. Treves, Linear partial differential equations with constant coefficients, Gordon and Breach, New York, 1966.

[Y] S. T. Yau, Seminar on differential geometry, Ann. of Math. Stud., no. 102, Princeton Univ. Press, 1982.

Department of Mathematics, Faculty of Science, Josai University, Saitama, Japan (Current address of Gen Nakamura)

Department of Mathematics, Mathematical Institute, University of Warwick, CovENTRY, CV4 7AL ENGLAND

Current address (Yoshiaki Maeda): Department of Mathematics, Faculty of Science and Technology, Keio University, Kanagawa, Japan 